\title{
DENSITY OF CHÂTELET SURFACES FAILING THE HASSE PRINCIPLE
}

\author{
by
}

R. de la Bretèche \& T.D. Browning

\begin{abstract}
Châtelet surfaces provide a rich source of geometrically rational surfaces which do not always satisfy the Hasse principle. Restricting attention to a special class of Châtelet surfaces, we investigate the frequency that such counter-examples arise over the rational numbers.
\end{abstract}

\section{Contents}

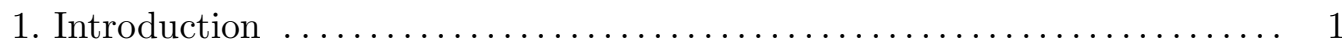

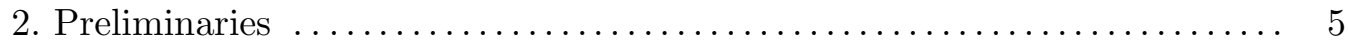

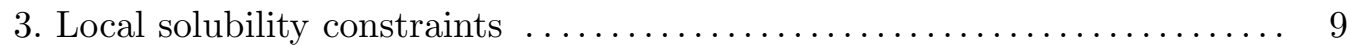

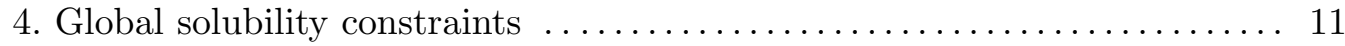

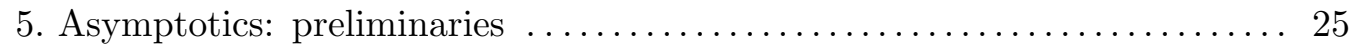

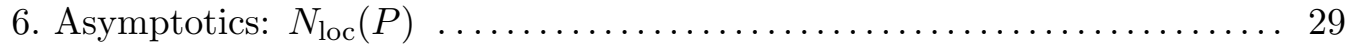

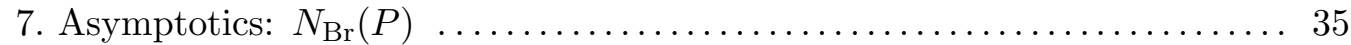

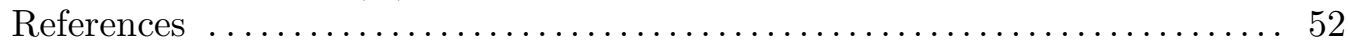

\section{Introduction}

A family of geometrically integral algebraic varieties defined over a number field $K$ is said to satisfy the Hasse principle if any variety in the family has a point in $K$ as soon as it has points in every completion of $K$. Quadrics are among the first examples of families satisfying this property. In dimension 2, Châtelet surfaces constitute a family of varieties for which the Hasse principle is known to fail in general. A Châtelet surface over $K$ is a proper smooth model of an affine surface

$$
Y^{2}-e Z^{2}=f(T)
$$

where $e \in K^{*}$ is not equal to a square in $K^{*}$ and $f \in K[T]$ is a separable polynomial of degree 3 or 4 . For these surfaces it follows from work of Colliot-Thélène, Sansuc and Swinnerton-Dyer [4] that all failures of the Hasse principle are accounted for by the Brauer-Manin obstruction, a cohomological obstruction based on the Brauer group of the surface. In particular it is known that the Hasse principle holds whenever $f$ is irreducible over $K$, which is the generic situation, or when $f$ has a linear factor over $K$. In the remaining case, when $f$ is a product

2010 Mathematics Subject Classification. - 14G05 (11D99, 11G35, 11G50). 
of two irreducible quadratic polynomials over $K$, counter-examples to the Hasse principle can arise.

In what follows we shall only consider Châtelet surfaces defined over $\mathbb{Q}$. A great deal of recent work has been directed at the quantitative arithmetic of rational varieties, the aim being to count $\mathbb{Q}$-rational points of bounded height on the variety, assuming that it contains a Zariski dense set of $\mathbb{Q}$-rational points. In this paper we seek instead to vary the varieties in a family and measure how often counter-examples to the Hasse principle emerge. In the setting of Châtelet surfaces, the main result in 4 implies that a random surface of the form (1.1) will satisfy the Hasse principle, and furthermore, we can only expect to find counter-examples when $f$ is a product of two quadratic polynomials over $\mathbb{Q}$, which are both irreducible over $\mathbb{Q}$.

For simplicity we will henceforth restrict our attention to Châtelet surfaces given by (1.1), for which $e=-1$ and $f$ factorises as a product of two diagonal quadratic polynomials over $\mathbb{Q}$. Thus, for any $(a, b, c, d) \in \mathbb{A}^{4}$, let $X_{a, b, c, d}$ denote the Châtelet surface defined by the equation

$$
Y^{2}+Z^{2}=\left(a T^{2}+b\right)\left(c T^{2}+d\right),
$$

with

$$
a b c d \neq 0, \quad a d-b c \neq 0 .
$$

The fact that the Brauer-Manin obstruction is the only obstruction to the Hasse principle for this family goes back to earlier work of Colliot-Thélène, Coray and Sansuc [3], wherein it is shown that $\operatorname{Br}\left(X_{a, b, c, d}\right) / \operatorname{Br}(\mathbb{Q}) \cong \mathbb{Z} / 2 \mathbb{Z}$ for any $(a, b, c, d) \in \mathbb{Q}^{4}$ such that (1.3) holds. (This work actually covers general Châtelet surfaces of the form (1.1) in which $f=q_{1} q_{2}$ for distinct quadratic polynomials $q_{1}, q_{2}$ that are irreducible over the base field.) Furthermore, in [3. Prop. C], a 1-parameter family of counter-examples to the Hasse principle over $\mathbb{Q}$ is exhibited. This family, which we will meet again in 84.5 , is given by $X_{1,1-k,-1, k}$ for any positive integer $k \equiv 3(\bmod 4)$. It generalises the particular case $k=3$ first discovered by Iskovskikh [7]. Our chief object is to give a finer quantitative treatment of this circle of ideas. As we vary over rational coefficients, producing surfaces $X_{a, b, c, d}$ that are defined over $\mathbb{Q}$, we will investigate the proportion of surfaces that

- have points everywhere locally,

- have $\mathbb{Q}$-rational points,

- fail the Hasse principle.

One of the issues that we shall need to address is how best to parameterise the Châtelet surfaces that are of interest to us.

Poonen and Voloch [9] have discussed similar questions in the setting of projective hypersurfaces $V \subset \mathbb{P}^{n}$ of degree $d$, assuming that $d, n \geqslant 2$ and $(d, n) \neq(2,2)$. Let $\Omega$ denote the set of valuations of $\mathbb{Q}$ and let $\mathbb{Q} v$ be the completion of $\mathbb{Q}$ at $v$, following the convention that $\mathbb{Q}_{\infty}=\mathbb{R}$. In [9. Thm. 3.6] it is shown that the proportion of $V$ that are everywhere locally soluble converges to an Euler product $c=\prod_{v \in \Omega} c_{v}>0$, where each $c_{v}$ is the proportion of $V$ for which $V\left(\mathbb{Q}_{v}\right) \neq \emptyset$. This is the exact analogue of our Theorem 1.2 for hypersurfaces. They conjecture, furthermore, that when $d \leqslant n$ (which implies that the hypersurface is generically Fano) the proportion of $V$ that have $\mathbb{Q}$-rational points should converge to this constant $c$. This is shown (see [9, Prop. 3.4]) to follow from the conjecture of Colliot-Thélène that the Brauer-Manin obstruction to the Hasse principle is the only one for smooth, proper, geometrically integral varieties over $\mathbb{Q}$ which are geometrically rationally connected. In fact, for non-singular hypersurfaces $V \subset \mathbb{P}^{n}$, with $n \geqslant 4$, the natural map $\operatorname{Br}(\mathbb{Q}) \rightarrow \operatorname{Br}(V)$ is an 
isomorphism. Thus the Brauer-Manin obstruction to the Hasse principle is generically empty when $n \geqslant 4$.

Bhargava 1 has recently undertaken an extensive investigation of hyperelliptic curves, where it is well-known that the Hasse principle can fail. Any hyperelliptic curve over $\mathbb{Q}$ of genus $g$ can be embedded in weighted projective space $\mathbb{P}(1,1, g+1)$, via an equation of the form

$$
T^{2}=F(Y, Z),
$$

where $F \in \mathbb{Z}[Y, Z]$ is a separable binary form of degree $2 g+2$. In this setting one gets different behaviour to that predicted for Fano hypersurfaces. It is shown in [1, Thm. 22] that for each $g \geqslant 1$, a positive proportion of hyperelliptic curves of genus $g$ over $\mathbb{Q}$, when ordered by height, fail the Hasse principle.

A form of degree $d$ in $n+1$ variables has $N=\left(\begin{array}{c}d+n \\ d\end{array}\right)$ possible coefficients. Poonen and Voloch use affine space $\mathbb{A}^{N}$ to parameterise hypersurfaces $V \subset \mathbb{P}^{n}$ of degree $d$, associating to each vector in $\mathbb{Z}^{N}$ a hypersurface $V$ defined over $\mathbb{Q}$. One disadvantage of this approach is that two different vectors may produce the same $V$. In our work it will be convenient to identify certain obvious choices of coefficients $(a, b, c, d)$ which lead to the same Châtelet surface $X_{a, b, c, d}$ in (1.2).

Let $(a, b, c, d) \in \mathbb{A}^{4}$ and $\left(a^{\prime}, b^{\prime}, c^{\prime}, d^{\prime}\right) \in \mathbb{A}^{4}$, with (1.3) holding for both sets of coefficients. The former gives rise to the Châtelet surface $X_{a, b, c, d}$ in (1.2) and, using different variables, the latter gives rise to the Châtelet surface $X_{a^{\prime}, b^{\prime}, c^{\prime}, d^{\prime}}$ given by

$$
Y^{\prime 2}+Z^{\prime 2}=\left(a^{\prime} T^{\prime 2}+b^{\prime}\right)\left(c^{\prime} T^{\prime 2}+d^{\prime}\right) .
$$

We will identify these surfaces, writing $X_{a^{\prime}, b^{\prime}, c^{\prime}, d^{\prime}}=X_{a, b, c, d}$, if there is matrix $M \in \mathrm{GL}_{2}(\mathbb{Q})$ and a scalar $m \in \mathbb{Q}^{*}$ such that

$$
\left(\begin{array}{c}
Y^{\prime} \\
Z^{\prime}
\end{array}\right)=M\left(\begin{array}{l}
Y \\
Z
\end{array}\right), \quad T^{\prime}=m T .
$$

In such a situation it is clear that the surfaces $X_{a^{\prime}, b^{\prime}, c^{\prime}, d^{\prime}}$ and $X_{a, b, c, d}$ really are identical.

We begin by noting that $X_{\lambda a, \lambda b, \lambda c, \lambda d}=X_{a, b, c, d}$ for any $\lambda \in \mathbb{Q}^{*}$, as can be seen by taking $M=\operatorname{diag}(\lambda, \lambda)$ and $m=1$ in (1.4). Thus we are led to consider the open set

$$
U=\left\{[a, b, c, d] \in \mathbb{P}^{3}:(1.3) \text { holds }\right\} .
$$

Similarly, we have $X_{\lambda^{2} a, \mu^{2} b, \lambda^{2} c, \mu^{2} d}=X_{a, b, c, d}$ for any $\lambda, \mu \in \mathbb{Q}^{*}$, as seen by taking $M=$ $\operatorname{diag}\left(\mu^{2}, \mu^{2}\right)$ and $m=\mu / \lambda$ in (1.4). Next, we let $N(\zeta)=\zeta_{1}^{2}+\zeta_{2}^{2}$ denote the norm of any $\zeta=\zeta_{1}+\mathrm{i} \zeta_{2} \in \mathbb{Q}(\mathrm{i})$. For $\xi, \eta \in \mathbb{Q}(\mathrm{i})^{*}$, we have $X_{N(\xi) a, N(\xi) b, N(\eta) c, N(\eta) d}=X_{a, b, c, d}$, where the transformation is given by

$$
M=\left(\begin{array}{cc}
\xi_{1} \eta_{1}-\xi_{2} \eta_{2} & -\left(\xi_{1} \eta_{2}+\xi_{2} \eta_{1}\right) \\
\xi_{1} \eta_{2}+\xi_{2} \eta_{1} & \xi_{1} \eta_{1}-\xi_{2} \eta_{2}
\end{array}\right)
$$

and $m=1$ in (1.4). In conclusion, we have produced an action of $\mathbb{G}_{m, \mathbb{Q}}^{2} \times R_{\mathbb{Q}(\mathrm{i}) / \mathbb{Q}}\left(\mathbb{G}_{m, \mathbb{Q}(\mathrm{i})}\right)^{2}$ on $U$, where $\mathbb{G}_{m, k}$ denotes the linear algebraic group Spec $K\left[T, T^{-1}\right]$ associated to a field $K$. Building on this, we note that $X_{(a, b, c, d)^{\varrho_{1}}}=X_{(a, b, c, d)^{\varrho_{2}}}=X_{a, b, c, d}$, where

$$
\varrho_{1}:(a, b, c, d) \mapsto(c, d, a, b), \quad \varrho_{2}:(a, b, c, d) \mapsto(b, a, d, c) .
$$

This leads to a further action on $U$ by the 0 -dimensional algebraic group $(\mathbb{Z} / 2 \mathbb{Z})^{2}$. We therefore obtain a group action $G \times U \rightarrow U$, where $G$ is the algebraic $\mathbb{Q}$-group

$$
G=\mathbb{G}_{m, \mathbb{Q}}^{2} \times R_{\mathbb{Q}(\mathrm{i}) / \mathbb{Q}}\left(\mathbb{G}_{m, \mathbb{Q}(\mathrm{i})}\right)^{2} \rtimes(\mathbb{Z} / 2 \mathbb{Z})^{2} .
$$


Given $u=[a, b, c, d] \in U$, the orbit of $u$ under $G(\mathbb{Q})$ produces the same surface $X_{a, b, c, d}$.

In our analysis we will restrict attention to elements of $\mathscr{M}=U(\mathbb{Q}) / G(\mathbb{Q})$. We will be interested in three basic subsets, given by

$$
\begin{aligned}
\mathscr{M}_{\mathrm{loc}} & =\left\{[a, b, c, d] \in \mathscr{M}: X_{a, b, c, d}\left(\mathbb{Q}_{v}\right) \neq \emptyset \forall v \in \Omega\right\}, \\
\mathscr{M}_{\text {glob }} & =\left\{[a, b, c, d] \in \mathscr{M}: X_{a, b, c, d}(\mathbb{Q}) \neq \emptyset\right\} \\
\mathscr{M}_{\mathrm{Br}} & =\mathscr{M}_{\mathrm{loc}} \backslash \mathscr{M}_{\text {glob }} .
\end{aligned}
$$

The elements of $\mathscr{M}_{\mathrm{Br}}$ are precisely the surfaces for which there is a non-empty BrauerManin obstruction to the Hasse principle. In $₫ 2$ we will choose representative coordinates in $\mathbb{Z}_{\text {prim }}^{4} /\{ \pm 1\}$ for the parameter space $\mathscr{M}$. In this way, to each point $u \in \mathscr{M}$ we can associate a height $H(u)$, which will turn out to be $\max \{|a|,|b|,|c|,|d|\}$ on $\mathbb{Z}_{\text {prim }}^{4} /\{ \pm 1\}$. With this in mind we wish to study the cardinalities

$$
N_{\text {loc } / \text { glob } / \mathrm{Br}}(P)=\#\left\{u \in \mathscr{M}_{\mathrm{loc} / \mathrm{glob} / \mathrm{Br}}: H(u) \leqslant P\right\},
$$

as $P \rightarrow \infty$. Our first result is the following.

Theorem 1.1. - We have $N_{\mathrm{loc}}(P)=\tau_{\mathrm{loc}} P^{4}+O\left(P^{4-1 / 8+\varepsilon}\right)$, for any $\varepsilon>0$, where if $\tau_{\mathrm{loc}, v}$ is the density of points in $U\left(\mathbb{Q}_{v}\right) / G\left(\mathbb{Q}_{v}\right)$ for which the associated surface has $\mathbb{Q}_{v}$-points then

$$
\tau_{\text {loc }}=\prod_{v \in \Omega} \tau_{\text {loc }, v} .
$$

Here, as throughout our work, the implied constant is allowed to depend at most on the choice of parameter $\varepsilon>0$. Theorem 1.1 will be established in $\$ 6$, where an explicit description of the factors $\tau_{\text {loc }, v}$ will appear. Once combined with an estimation of the total number $N(P)$ of elements in $\mathscr{M}$ with height at most $P$, we will also establish the following result.

Theorem 1.2. - We have

where

$$
\lim _{P \rightarrow \infty} \frac{N_{\mathrm{loc}}(P)}{N(P)}=\frac{33257}{39168} \prod_{p \equiv 3(\bmod 4)}\left(1-\frac{6-\frac{9}{p^{2}}+\frac{4}{p^{4}}}{p^{4} b_{p}}\right),
$$

$$
b_{p}=\left(1+\frac{1}{p}\right)^{2}\left(1+\frac{2}{p}+\frac{3}{p^{2}}+\frac{4}{p^{3}}-\frac{4}{p^{5}}\right) .
$$

In particular $83.3 \%$ of the elements of $\mathscr{M}$ are everywhere locally soluble.

In $\$ 4$ we will characterise when a surface $X=X_{a, b, c, d}$ in $\mathscr{M}_{\text {loc }}$ actually belongs to $\mathscr{M}_{\text {glob }}$. The Brauer group $\operatorname{Br}(X)$ will play a fundamental rôle in this analysis, for which purpose we recall some basic facts here. For any field $K \supseteq \mathbb{Q}$, each element $\mathscr{A} \in \operatorname{Br}(X)$ gives rise to an evaluation map ev $\mathscr{A}: X(K) \rightarrow \operatorname{Br}(K)$. Class field theory gives the exact sequence

$$
0 \longrightarrow \operatorname{Br}(\mathbb{Q}) \longrightarrow \bigoplus_{v} \operatorname{Br}\left(\mathbb{Q}_{v}\right) \stackrel{\sum_{v} \operatorname{inv}_{v}}{\longrightarrow} \mathbb{Q} / \mathbb{Z} \longrightarrow 0
$$

For any $\mathscr{A} \in \operatorname{Br}(X)$ we then have the commutative diagram

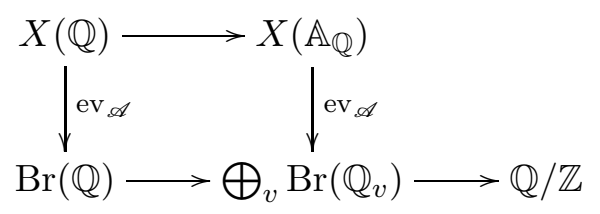


where $\mathbb{A}_{\mathbb{Q}}$ denotes the adèles and $X\left(\mathbb{A}_{\mathbb{Q}}\right)=\prod_{v} X\left(\mathbb{Q}_{v}\right)$. Let $\Theta_{\mathscr{A}}: X\left(\mathbb{A}_{\mathbb{Q}}\right) \rightarrow \mathbb{Q} / \mathbb{Z}$ denote the composed map. Then it follows that $X(\mathbb{Q}) \subset \operatorname{ker} \Theta_{\mathscr{A}}$ for all $\mathscr{A} \in \operatorname{Br}(X)$. We write

$$
X\left(\mathbb{A}_{\mathbb{Q}}\right)^{\operatorname{Br}(X)}=\bigcap_{\mathscr{A} \in \operatorname{Br}(X)} \operatorname{ker} \Theta_{\mathscr{A}}
$$

In our setting, $\operatorname{Br}(X) / \operatorname{Br}(\mathbb{Q}) \cong \mathbb{Z} / 2 \mathbb{Z}$ has order 2 and so $\operatorname{inv}_{v}\left(\operatorname{ev}_{\mathscr{A}}\left(M_{v}\right)\right)$ takes the values 0 or $1 / 2$ in $\mathbb{Q} / \mathbb{Z}$. An obstruction to the Hasse principle arises if and only if $X\left(\mathbb{A}_{\mathbb{Q}}\right)^{\operatorname{Br}(X)}=\emptyset$, that is to say, if and only if for any $\left(M_{v}\right) \in X\left(\mathbb{A}_{\mathbb{Q}}\right)$ there exists $\mathscr{A} \in \operatorname{Br}(X)$ such that

$$
\sum_{v} \operatorname{inv}_{v}\left(\operatorname{ev}_{\mathscr{A}}\left(M_{v}\right)\right)=\frac{1}{2} \text {. }
$$

Moreover, a generator for $\operatorname{Br}(X) / \operatorname{Br}(\mathbb{Q})$ is given by the quaternion algebra $\left(-1, a T^{2}+b\right)$ on (1.2). Fundamental to our work is the observation that (1.8) is impossible if there exists a valuation $v$ for which $\operatorname{inv}_{v}\left(\operatorname{ev}_{\mathscr{A}}\left(M_{v}\right)\right)$ takes both values 0 and $1 / 2$, as a function of $M_{v}$. Thus, in order to obtain counter-examples to the Hasse principle, it will be necessary for $\operatorname{inv}_{v}\left(\operatorname{ev}_{\mathscr{A}}\left(M_{v}\right)\right)$ to be constant for every valuation $v$.

In $\$ 7$ we shall build on $\$ 4$ to establish the following asymptotic formula for the density of surfaces which provide counter-examples to the Hasse principle.

Theorem 1.3. - Let $\varepsilon>0$. There exists $\tau_{\mathrm{Br}}>0$ such that

$$
N_{\mathrm{Br}}(P)=\frac{\tau_{\mathrm{Br}} P^{4}}{(\log P)^{1 / 4}}+O\left(\frac{P^{4}}{(\log P)^{3 / 4-\varepsilon}}\right) .
$$

It follows from Theorems 1.1 and 1.3 that $N_{\text {glob }}(P)$ satisfies the asymptotic behaviour predicted by Poonen and Voloch [9] for Fano hypersurfaces. Moreover, once coupled with Theorem [1.2, Theorem 1.3 shows that $83.3 \%$ of the elements of $\mathscr{M}$ are soluble over $\mathbb{Q}$. It should be stressed that our argument provides a completely explicit algorithm for determining whether or not a given Châtelet surface $X_{a, b, c, d}$ of the form (1.2) gives a counter-example to the Hasse principle, without explicitly needing to work with elements of $\operatorname{Br}\left(X_{a, b, c, d}\right) / \operatorname{Br}(\mathbb{Q})$.

Acknowledgements. - The authors are grateful to Jean-Louis Colliot-Thélène and Étienne Fouvry for suggesting this problem to us, to Pierre Le Boudec and the anonymous referee for comments on an earlier draft and to Tim Dokchitser for help with verifying numerically the proof of Lemma 6.2. While working on this paper the first author was supported by an IUF Junior and ANR project (PEPR), while the second author was supported by ERC grant 306457.

\section{Preliminaries}

We will need to choose representative coordinates for the parameter space $\mathscr{M}$ that we met in the introduction. Two sets that will feature heavily in our work are

$$
\mathscr{A}=\left\{n \in \mathbb{N}: \mu(n)^{2}=1\right\}, \quad \mathscr{B}=\{n \in \mathscr{A}: p \mid n \Rightarrow p \equiv 3(\bmod 4)\},
$$

where $\mu$ denotes the Möbius function. Let $\mathbb{Z}_{\text {prim }}^{4}$ denote the set of relatively prime 4 -tuples of integers. We define

$$
S_{\text {tot }}=\left\{(a, b, c, d) \in \mathbb{Z}_{\text {prim }}^{4} /\{ \pm 1\}: \begin{array}{l}
a d-b c \neq 0, a b c d \neq 0 \\
\operatorname{gcd}(a, c), \operatorname{gcd}(b, d) \in \mathscr{A} \\
\operatorname{gcd}(a, b), \operatorname{gcd}(c, d) \in \mathscr{B}
\end{array}\right\} .
$$


It is clear, in view of the invariance under the action of the maps (1.5), that we have a $1: 4$ bijection between $\mathscr{M}$ and our set of representatives $S_{\text {tot }}$.

When it comes to determining whether or not elements of $S_{\text {tot }}$ produce surfaces $X_{a, b, c, d}$ with 2 -adic points, a tedious number of subcases arise, depending on the residue classes modulo 4 of the coefficients. In order to reduce the number of cases that need to be considered we note that for any $(a, b, c, d) \in S_{\text {tot }}$, we have $(a, b) \in\{(1,1),(1,0),(0,1)\}(\bmod 2)$. But $S_{\text {tot }}$ is left invariant under the action of the map $\varrho_{2}$ defined in (1.5). Hence we may write

$$
S_{\text {tot }}=\left\{(a, b, c, d) \in S_{\text {tot }}: 2 \nmid a\right\} \sqcup\left\{(a, b, c, d) \in S_{\text {tot }}: 2 \nmid a, 2 \mid b\right\} .
$$

The elements of $S_{\text {tot }}$ have non-zero coordinates. Since we identify $(a, b, c, d)$ with $-(a, b, c, d)$ in $S_{\text {tot }}$, it will suffice to take representative coordinates in which $a>0$. The sets in which we are interested therefore take the shape

$$
S_{\text {tot }}^{(\iota)}=\left\{(a, b, c, d) \in \mathbb{Z}_{\text {prim }}^{4}: \begin{array}{l}
a>0, b c d(a d-b c) \neq 0 \\
\operatorname{gcd}(a, c), \operatorname{gcd}(b, d) \in \mathscr{A} \\
\operatorname{gcd}(a, b), \operatorname{gcd}(c, d) \in \mathscr{B} \\
\left(a, 2^{\iota} b\right) \equiv(1,0)(\bmod 2)
\end{array}\right\},
$$

for $\iota \in\{0,1\}$. Among the elements of these sets we will be interested primarily in those coefficients which give rise to Châtelet surfaces $X_{a, b, c, d}$ which have points everywhere locally, or points globally. Let us therefore define the sets

$$
\begin{aligned}
S_{\text {loc }}^{(\iota)} & =\left\{(a, b, c, d) \in S_{\text {tot }}^{(\iota)}: X_{a, b, c, d}\left(\mathbb{Q}_{v}\right) \neq \emptyset \forall v \in \Omega\right\}, \\
S_{\text {glob }}^{(\iota)} & =\left\{(a, b, c, d) \in S_{\text {tot }}^{(\iota)}: X_{a, b, c, d}(\mathbb{Q}) \neq \emptyset\right\},
\end{aligned}
$$

for $\iota \in\{0,1\}$.

We proceed to introduce some further notation. Given $(a, b, c, d) \in S_{\text {tot }}^{(\iota)}$, we define the binary quadratic forms

$$
Q_{1}(U, V)=a U^{2}+b V^{2}, \quad Q_{2}(U, V)=c U^{2}+d V^{2} .
$$

Let $m=\operatorname{gcd}(a, b)$ and $n=\operatorname{gcd}(c, d)$. Then $m, n \in \mathscr{B}$, with $\operatorname{gcd}(m, n)=1$. We henceforth write

$$
Q_{1}^{\prime}(U, V)=a^{\prime} U^{2}+b^{\prime} V^{2}, \quad Q_{2}^{\prime}(U, V)=c^{\prime} U^{2}+d^{\prime} V^{2},
$$

where $(a, b)=m\left(a^{\prime}, b^{\prime}\right)$ and $(c, d)=n\left(c^{\prime}, d^{\prime}\right)$. In particular we have $\operatorname{gcd}\left(a^{\prime}, b^{\prime}\right)=\operatorname{gcd}\left(c^{\prime}, d^{\prime}\right)=1$ and

$$
\begin{aligned}
d^{\prime} Q_{1}^{\prime}(U, V)-b^{\prime} Q_{2}^{\prime}(U, V) & =\Delta^{\prime} U^{2}, \\
-c^{\prime} Q_{1}^{\prime}(U, V)+a^{\prime} Q_{2}^{\prime}(U, V) & =\Delta^{\prime} V^{2},
\end{aligned}
$$

where $\Delta^{\prime}=a^{\prime} d^{\prime}-b^{\prime} c^{\prime}$.

Returning to the family of surfaces $X_{a, b, c, d}$ arising as proper smooth models of (1.2), in gauging solubility over the completed field $\mathbb{Q}_{v}$, for any $v \in \Omega$, it will suffice to work with a non-empty Zariski open subset $W$ of $X_{a, b, c, d}$. Indeed, according to [3. Lemme 3.1.2], the set $W\left(\mathbb{Q}_{v}\right)$ has dense image in $X_{a, b, c, d}\left(\mathbb{Q}_{v}\right)$ for the topology defined by the natural topology of $\mathbb{Q}_{v}$. In this way we see that it suffices to examine the local solubility of the affine equation

$$
0 \neq Y^{2}+Z^{2}=Q_{1}(U, V) Q_{2}(U, V),
$$

where $Q_{1}, Q_{2}$ are as in (2.5). Our first order of business is a precise characterisation of when an element can locally be written as the sum of two squares. 
Let $v \in \Omega$ and let $\Sigma_{v}$ denote the non-zero elements in $\mathbb{Q}_{v}$ which can be written as a sum of two squares in $\mathbb{Q}_{v}$. Firstly we note that $\Sigma_{\infty}=\mathbb{R}_{>0}$. When $v$ is a prime $p \equiv 1(\bmod 4)$ then $\Sigma_{p}=\mathbb{Q}_{p}^{*}$. Next when $v$ is a prime $p \equiv 3(\bmod 4)$ then $\Sigma_{p}$ is the set of $t \in \mathbb{Q}_{p}^{*}$ for which the $p$-adic valuation $v_{p}(t)$ of $t$ is even. Finally when $v=2$ we have $\Sigma_{2}=\mathscr{D}$, where

$$
\mathscr{D}=\left\{2^{n}(1+4 m): m \in \mathbb{Z}_{2}, n \in \mathbb{Z}\right\} .
$$

Frequent use will be made of this characterisation in $\S 3$ and $\$ 4$

We will reserve the letter $p$ for denoting a prime number. Our work will involve various standard arithmetic functions, including $\omega(n)=\sum_{p \mid n} 1$, the generalised divisor function $\tau_{k}(n)=\sum_{n=d_{1} \cdots d_{k}} 1$ and the Euler totient function $\varphi$. In addition to this we will also meet the function

$$
\varphi_{\delta}^{*}(n)=\prod_{p \mid n}\left(1-\frac{1}{p^{\delta}}\right)
$$

for any $\delta>0$, so that $\varphi_{1}^{*}(n)=\varphi(n) / n$. Note that $1 /(\log \log n) \ll \varphi_{1}^{*}(n) \leqslant 1$. Finally, let

$$
\psi(n)=\prod_{p \mid n}\left(1+\frac{1}{p}\right)
$$

We will typically abbreviate $\varphi_{1}^{*}(n)$ by $\varphi^{*}(n)$ and $\tau_{2}$ by $\tau$.

We will also require an estimate for the average order of the divisor function, as it ranges over the values of a binary linear form. A crucial aspect of the following result is the uniformity in the coefficients of the linear form that it enjoys.

Lemma 2.1. - Let $\mathbf{a} \in \mathbb{Z}^{2}$ with $a_{1} a_{2} \neq 0$, let $V_{1}, V_{2} \geqslant 2$ and let $\varepsilon>0$. We have

$$
\sum_{\substack{v_{1} \leqslant V_{1} \\ v_{2} \leqslant V_{2}}} \tau\left(\left|a_{1} v_{1}+a_{2} v_{2}\right|\right) \ll \tau\left(\operatorname{gcd}\left(a_{1}, a_{2}\right)\right) \psi\left(a_{1} a_{2}\right) V_{1} V_{2} \log \left(V_{1} V_{2}\right)+\frac{\max _{i}\left|a_{i}\right|^{\varepsilon} \max _{i}\left\{V_{i}\right\}^{1+\varepsilon}}{\min _{i}\left\{V_{i}\right\}},
$$

where the implied constant depends at most on $\varepsilon$.

Proof. - Suppose without loss of generality that $V_{1} \geqslant V_{2}$. The bound follows from previous work of the authors [2, Thm. 1] when $V_{2} \gg \max _{i}\left|a_{i}\right|^{\varepsilon / 4} V_{1}^{\varepsilon / 4}$. Alternatively it follows from the trivial bound $\tau(n)=O\left(n^{\varepsilon}\right)$.

We close this section with a modified result of Le Boudec [8, Lemma 2], concerning pairs of integers constrained to satisfy congruences modulo $q$ and modulo $r$, with $\operatorname{gcd}(q, r)=1$. We are interested in the cardinality

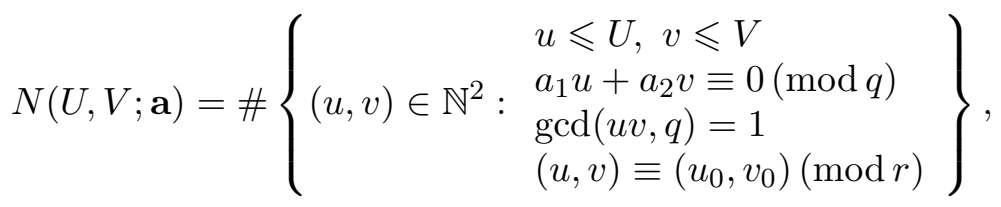

for suitable $\mathbf{a}, u_{0}, v_{0}, U, V$. The following result allows us to approximate $N(U, V ; \mathbf{a})$ by the expected main term.

Lemma 2.2. - Let $\mathbf{a} \in \mathbb{Z}^{2}$ with $a_{1} a_{2} \neq 0$ and $\operatorname{gcd}\left(a_{1} a_{2} r, q\right)=1$, let $\left(u_{0}, v_{0}\right) \in \mathbb{Z}^{2}$ and let $U, V \geqslant 1$. We have

$$
\left|N(U, V ; \mathbf{a})-\frac{\varphi(q) U V}{q^{2} r^{2}}\right| \ll \tau_{3}(q)\left(\frac{U+V}{q}+(\log q r)^{3}\right)+E(\mathbf{a}),
$$


where

$$
E(\mathbf{a})=\sum_{d \mid q} d \sum_{\substack{0<|m|,|n| \leqslant q r / 2 \\ m a_{2}-n a_{1} \equiv 0(\bmod d)}} \frac{1}{|m n|}
$$

Proof. - As we have mentioned, this result is based on work of Le Boudec [8, Lemma 2], which corresponds to $r=1$ and general closed sets $\mathscr{I}, \mathscr{J} \subset \mathbb{R}$ in place of $[1, U]$ and $[1, V]$. We have decided to include a sketch of the proof for completeness, the key idea being to break the $(u, v)$ into congruence classes modulo $q r$ and then use the orthogonality of additive characters $e_{q r}(\cdot)$ to detect these congruences.

Since $q$ and $r$ are coprime, there exist $\bar{q}, \bar{r} \in \mathbb{Z}$ such that $q \bar{q}+r \bar{r}=1$. We therefore have

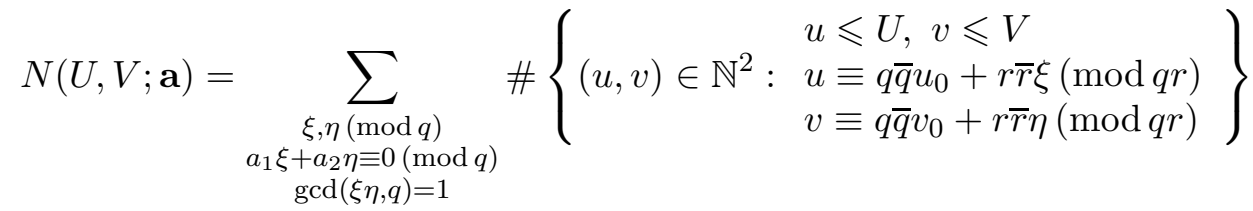

$$
\begin{aligned}
& =\frac{1}{q^{2} r^{2}} \sum_{m, n(\bmod q r)} S(m, n) \sum_{u \leqslant U} e_{q r}(-m u) \sum_{v \leqslant V} e_{q r}(-n v),
\end{aligned}
$$

where

$$
S(m, n)=e_{r}\left(m \bar{q} u_{0}+n \bar{q} v_{0}\right) \sum_{\substack{\xi, \eta(\bmod q) \\ a_{1} \xi+a_{2} \eta \equiv 0(\bmod q) \\ \operatorname{gcd}(\xi \eta, q)=1}} e_{q}(m \bar{r} \xi+n \bar{r} \eta) .
$$

Here, recalling that $\operatorname{gcd}\left(a_{1} a_{2}, q\right)=1$, we have

$$
S(m, n)=e_{r}\left(m \bar{q} u_{0}+n \bar{q} v_{0}\right) c_{q}\left(m a_{2}-n a_{1}\right),
$$

where $c_{q}(k)$ is the Ramanujan sum, satisfying $\left|c_{q}(k)\right| \leqslant \operatorname{gcd}(k, q)$. In particular $S(0, n)$ and $S(m, 0)$ are seen to be independent of $a_{1}, a_{2}$.

We denote by $N(U, V)$ the contribution from $m=0$ or $n=0$. Then bounding the sums over $u, v$ using the standard bound for linear exponential sums, we see that

$$
N(U, V ; \mathbf{a})-N(U, V) \ll \sum_{0<|m|,|n| \leqslant q r / 2} \frac{|S(m, n)|}{|m n|} \ll E(\mathbf{a}),
$$

in the notation of the lemma. Summing over $a_{1} \in(\mathbb{Z} / q \mathbb{Z})^{*}$ we deduce that

$$
\begin{aligned}
\sum_{a_{1}} N(U, V ; \mathbf{a})-\varphi(q) N(U, V) & \ll \sum_{d \mid q} d \sum_{0<|m|,|n| \leqslant q r / 2} \frac{1}{|m n|} \sum_{\substack{a_{1}(\bmod q) \\
\operatorname{gcd}\left(a_{1}, q\right)=1 \\
m a_{2}-n a_{1} \equiv 0(\bmod d)}} 1 \\
& \ll \sum_{d \mid q} d \sum_{h \mid d} \frac{1}{h^{2}} \sum_{\substack{0<\left|m^{\prime}\right|,\left|n^{\prime}\right| \leqslant q r /(2 h) \\
\operatorname{gcd}\left(m^{\prime}, n^{\prime}, d / h\right)=1}} \frac{q h}{d\left|m^{\prime} n^{\prime}\right|} \\
& \ll q \tau_{3}(q)(\log q r)^{2} .
\end{aligned}
$$


Moreover, it is clear that

$$
\begin{aligned}
& \sum_{a_{1}} N(U, V ; \mathbf{a})=\#\left\{\begin{array}{ll}
(u, v) \in \mathbb{N}^{2}: & u \leqslant U, v \leqslant V \\
& (u, v) \equiv\left(u_{0}, v_{0}\right)(\bmod r)
\end{array}\right\} \\
& =\frac{\varphi^{*}(q)^{2} U V}{r^{2}}+O(\tau(q)(U+V)) .
\end{aligned}
$$

Note that $\varphi^{*}(q) / \varphi(q)=1 / q$. Combining this with (2.13) we swiftly arrive at the statement of the lemma.

\section{Local solubility constraints}

As we have seen, in order to determine whether or not $X_{a, b, c, d}$ is soluble over $\mathbb{Q}_{v}$, for any $v \in \Omega$, it will suffice to work with the equation (2.8). Furthermore, when considering solubility over $\mathbb{Q}_{p}$, it suffices to establish the existence of solutions $(y, z, u, v)$ for which $u, v \in \mathbb{Z}_{p}$ are coprime. In order to summarise the local solubility criterion for $X_{a, b, c, d}$ it will be convenient to introduce a new symbol. For any odd prime $p$ and any $n \in \mathbb{Z}$ with $v_{p}(n)=\nu$, we set

$$
\left[\frac{n}{p}\right]= \begin{cases}\left(\frac{n / p^{\nu}}{p}\right), & \text { if } 2 \mid \nu, \\ 1, & \text { if } 2 \nmid \nu,\end{cases}
$$

where $(\dot{\bar{p}})$ is the usual Legendre symbol. This symbol takes values in $\{ \pm 1\}$. Finally, let $\sigma(t) \in\{-,+\}$ denote the sign of any real number $t$, which we extend to vectors in the obvious way. We may now record the following result, in which $m=\operatorname{gcd}(a, b)$ and $n=\operatorname{gcd}(c, d)$.

Lemma 3.1. - Let $(a, b, c, d) \in S_{\text {tot }}^{(\iota)}$. Then the following hold:

(i) $X_{a, b, c, d}(\mathbb{R}) \neq \emptyset$ if and only if $\sigma(a, b, c, d) \neq(+,+,-,-)$;

(ii) $X_{a, b, c, d}\left(\mathbb{Q}_{p}\right) \neq \emptyset$ if $v_{p}(m n)=0$;

(iii) if $v_{p}(m n)=1$ for $p \equiv 3(\bmod 4)$ then $X_{a, b, c, d}\left(\mathbb{Q}_{p}\right) \neq \emptyset$ if and only if

$-v_{p}\left(a^{\prime}\right)=v_{p}\left(c^{\prime}\right)$ or $v_{p}\left(b^{\prime}\right)=v_{p}\left(d^{\prime}\right)$ only when these $p$-adic orders are zero,

- either $\left[\frac{-a^{\prime} b^{\prime}}{p}\right]=1$ or $\left[\frac{-c^{\prime} d^{\prime}}{p}\right]=1$;

(iv) $X_{a, b, c, d}\left(\mathbb{Q}_{2}\right) \neq \emptyset$ if and only if $\exists$ coprime $u, v \in \mathbb{Z}_{2}$ such that $Q_{1}(u, v) Q_{2}(u, v) \in \mathscr{D}$.

One recalls here that $m, n \in \mathscr{B}$, with $\operatorname{gcd}(m, n)=1$. Thus $v_{p}(m n) \leqslant 1$ for any prime $p$ and $X_{a, b, c, d}\left(\mathbb{Q}_{p}\right) \neq \emptyset$ whenever $p \equiv 1(\bmod 4)$.

Proof of Lemma 3.1. - That the conditions are necessary is self-evident. Turning to sufficiency, we begin by establishing (i), noting that $a>0$ for any $(a, b, c, d) \in S_{\text {tot }}^{(\iota)}$. The case in which $a c$ or $b d$ is positive is trivial, so we study only the case $a c<0$ and $b d<0$, with (i) being satisfied. In this case $b c>0$ and $a d>0$, whence

$$
Q_{1} Q_{2}(\sqrt{a d+b c}, \sqrt{-2 a c})=-a c(a d-b c)^{2}>0 .
$$

Condition (iv) is a direct consequence of the criterion for solubility of $X_{a, b, c, d}$ over $\mathbb{Q}_{2}$. Solubility over $\mathbb{Q}_{p}$ is automatic for $p \equiv 1(\bmod 4)$.

It therefore remains to consider solubility over $\mathbb{Q}_{p}$ when $p \equiv 3(\bmod 4)$. For ease of notation we will henceforth write $Q_{i}^{\prime}$ for $Q_{i}^{\prime}(u, v)$. We claim that there exist coprime $u, v \in \mathbb{Z}_{p}$ such that $v_{p}\left(Q_{1}^{\prime} Q_{2}^{\prime}\right)=0$. This will clearly suffice to show that $X_{a, b, c, d}\left(\mathbb{Q}_{p}\right)$ is non-empty when $v_{p}(m n)=0$, as required for condition (ii). Recall that $\operatorname{gcd}\left(a^{\prime}, b^{\prime}\right)=1$ and $\operatorname{gcd}\left(c^{\prime}, d^{\prime}\right)=1$. If 
$p \nmid a^{\prime} c^{\prime}$ then the claim is satisfied by taking $(u, v)=(1,0)$. Similarly, if $p \nmid b^{\prime} d^{\prime}$ then we may take $(u, v)=(0,1)$. The remaining cases to consider are when $p \mid \operatorname{gcd}\left(b^{\prime}, c^{\prime}\right)$ with $p \nmid a^{\prime} d^{\prime}$, and lastly when $p \mid \operatorname{gcd}\left(a^{\prime}, d^{\prime}\right)$ with $p \nmid b^{\prime} c^{\prime}$. For both of these the choice $(u, v)=(1,1)$ is satisfactory, which thereby concludes the proof of the claim.

For the remainder of the proof we suppose that $v_{p}(m n)=1$, with $p \equiv 3(\bmod 4)$. We wish to show that under the hypotheses of the lemma we can always find coprime $u, v \in \mathbb{Z}_{p}$ such that precisely one of $v_{p}\left(Q_{1}^{\prime}\right)$ or $v_{p}\left(Q_{2}^{\prime}\right)$ is odd. This will then imply that $v_{p}\left(Q_{1}(u, v) Q_{2}(u, v)\right)$ is even as required for condition (iii).

Suppose first that $p \nmid a^{\prime} b^{\prime} c^{\prime} d^{\prime}$. Then by hypothesis one of either $-a^{\prime} b^{\prime}$ or $-c^{\prime} d^{\prime}$ is a nonzero square modulo $p$. There are two cases to consider. Suppose first that $\left(\frac{-a^{\prime} b^{\prime}}{p}\right)=1$ but $\left(\frac{-c^{\prime} d^{\prime}}{p}\right)=-1$. We can find coprime $u, v \in \mathbb{Z}_{p}$ such that $v_{p}\left(Q_{1}^{\prime}\right)=1$. On the other hand $v_{p}\left(Q_{2}^{\prime}\right)$ is even since $-c^{\prime} d^{\prime}$ is not a square modulo $p$. Next suppose that $\left(\frac{-a^{\prime} b^{\prime}}{p}\right)=\left(\frac{-c^{\prime} d^{\prime}}{p}\right)=1$, with $v_{p}\left(\Delta^{\prime}\right)=\kappa \geqslant 0$. We may choose coprime $u, v \in \mathbb{Z}_{p}$ so that $v_{p}\left(Q_{1}^{\prime}\right)=\kappa+1$. Then if $v_{p}\left(Q_{2}^{\prime}\right)=j$ one sees that $p^{\min \{j, \kappa+1\}}$ is a divisor of $\Delta^{\prime} \operatorname{gcd}(u, v)^{2}$ by (2.7). It therefore follows from (2.7) that $v_{p}\left(Q_{2}^{\prime}\right)=\kappa$, as required.

We now suppose that $p \mid a^{\prime} b^{\prime}$ but $p \nmid c^{\prime} d^{\prime}$. If $\left(\frac{-c^{\prime} d^{\prime}}{p}\right)=1$ then it is clear that we can find $u, v \in \mathbb{Z}_{p}$, with $p \nmid u v$, such that $v_{p}\left(Q_{2}^{\prime}\right)$ is odd. Moreover, we will have $p \nmid Q_{1}^{\prime}$ since $p$ divides precisely one of $a^{\prime}$ or $b^{\prime}$. If $\left(\frac{-c^{\prime} d^{\prime}}{p}\right)=-1$ then $v_{p}\left(Q_{2}^{\prime}\right)=0$ for any coprime $u, v \in \mathbb{Z}_{p}$. Suppose $p \mid a^{\prime}$, with $a^{\prime}=p^{\alpha} a^{\prime \prime}$ and $p \nmid a^{\prime \prime} b^{\prime}$. If $\alpha$ is odd then we can ensure that $v_{p}\left(Q_{1}^{\prime}\right)$ odd by taking $(u, v)=(1,0)$. Alternatively, if $\alpha$ is even then $\left(\frac{-a^{\prime \prime} b^{\prime}}{p}\right)=1$ by hypothesis. Hence we can force $v_{p}\left(Q_{1}^{\prime}\right)$ to be odd by considering solutions of the form $\left(u, p^{\alpha / 2} v^{\prime}\right)$. The case in which $p \nmid a^{\prime} b^{\prime}$ but $p \mid c^{\prime} d^{\prime}$ follows by symmetry.

It remains to consider the possibility $p \mid a^{\prime} b^{\prime}$ and $p \mid c^{\prime} d^{\prime}$. We will consider the cases $p \mid\left(a^{\prime}, d^{\prime}\right)$ or $p \mid\left(a^{\prime}, c^{\prime}\right)$, the remaining cases following by symmetry. Suppose first that $a^{\prime}=p^{\alpha} a^{\prime \prime}$ and $d^{\prime}=p^{\delta} d^{\prime \prime}$ with $p \nmid a^{\prime \prime} b^{\prime} c^{\prime} d^{\prime \prime}$. If $\alpha$ (resp. $\delta$ ) is odd then we are done on taking $(u, v)=(1,0)$ (resp. $(u, v)=(0,1))$. Suppose that $\alpha$ and $\delta$ are both even. Then our hypothesis ensures that one of the Legendre symbols $\left(\frac{-a^{\prime \prime} b^{\prime}}{p}\right)$ or $\left(\frac{-c^{\prime} d^{\prime \prime}}{p}\right)$ is equal to 1 . Supposing without loss of generality that it is the former, we easily make $v_{p}\left(Q_{1}^{\prime}\right)$ odd with $v_{p}\left(Q_{2}^{\prime}\right)=0$. Turning to the case $p \mid\left(a^{\prime}, c^{\prime}\right)$, we write $a^{\prime}=p^{\alpha} a^{\prime \prime}$ and $c^{\prime}=p^{\gamma} c^{\prime \prime}$ with $p \nmid a^{\prime \prime} b^{\prime} c^{\prime \prime} d^{\prime}$ and $\alpha, \gamma$ not both even. If $\alpha, \gamma$ are of opposite parities then the situation is easy and we can proceed by taking $(u, v)=(1,0)$. Finally, if $\alpha, \gamma$ are both odd then by hypothesis they must be unequal. Suppose without loss of generality that $\alpha=2 \alpha^{\prime}+1<\gamma$. Then in fact $\gamma>\alpha+1$. Taking $(u, v)=\left(1, p^{\alpha^{\prime}+1}\right)$ we easily deduce that $v_{p}\left(Q_{1}^{\prime}\right)=2 \alpha^{\prime}+1$ and $v_{p}\left(Q_{2}^{\prime}\right)=2 \alpha^{\prime}+2$, as required. This concludes the proof of the lemma.

Lemma 3.1 gives us a means of characterising the elements of $S_{\text {loc }}^{(\iota)}$, in the notation of (2.4). Before proceeding to the proof of Theorem 1.1, we will need a finer description of case (iv) in Lemma 3.1. This will depend intimately on the possible residue classes of $(a, b)$ modulo 4 , with $a$ odd. The various constraints are obtained by taking $(A, B, C, D)=(a, b, c, d)$ in the second part of Lemmas 4.8 4.15 in $\$ 4.4$, where the description of $T_{\text {tot }}^{(2)}$ exactly provides necessary and sufficient conditions for the non-nullity of $X_{a, b, c, d}\left(\mathbb{Q}_{2}\right)$. 


\section{Global solubility constraints}

Throughout this section we will assume familiarity with the notation introduced in 92 . Among the set of $(a, b, c, d) \in S_{\text {loc }}^{(\iota)}$ we require a means of sifting for those $(a, b, c, d)$ such that $X_{a, b, c, d}$ actually has points in $\mathbb{Q}$. Our principal tool comes from the theory of descent, as formulated by Colliot-Thélène, Coray and Sansuc [3, Cor. 5.2]. This ensures that given any $(a, b, c, d) \in S_{\text {loc }}^{(\iota)}$, we have $(a, b, c, d) \in S_{\text {glob }}^{(\iota)}$ if and only if there exists $\mathbf{e}=\left(e_{1}, e_{2}\right) \in \mathbb{Q}^{2}$, with $e_{1} e_{2}=1$, such that the smooth variety

$$
\left\{\begin{array}{l}
0 \neq Y_{1}^{2}+Z_{1}^{2}=e_{1}\left(a T^{2}+b\right), \\
0 \neq Y_{2}^{2}+Z_{2}^{2}=e_{2}\left(c T^{2}+d\right),
\end{array}\right.
$$

has points in $\mathbb{Q}_{w}$ for every $w \in \Omega$. This in turn is equivalent to the existence of $\mathbf{e} \in \mathbb{Q}^{2}$, with $e_{1} e_{2}=m n$, such that the corresponding pair of equations with $a, b, c, d$ replaced by $a^{\prime}, b^{\prime}, c^{\prime}, d^{\prime}$ is everywhere locally soluble. Note that, by [3. Lemme 3.1.2], we see that in checking local solubility for the pair of equations it suffices to replace $a^{\prime} T^{2}+b^{\prime}$ and $c^{\prime} T^{2}+d^{\prime}$ by $Q_{1}^{\prime}(U, V)$ and $Q_{2}^{\prime}(U, V)$, respectively, as given by (2.6).

We proceed to simplify the set of allowable e somewhat. On carrying out a suitable change of variables it suffices, without loss of generality, to consider the existence of $\mathbf{e} \in \mathbb{Q}^{2}$ for which

$$
v_{p}\left(e_{i}\right) \in \begin{cases}\{0\}, & \text { if } p \neq \equiv 3(\bmod 4), \\ \{0,-1,1\}, & \text { if } p \equiv 3(\bmod 4),\end{cases}
$$

for $i=1,2$. Recall that $m, n \in \mathscr{B}$, with $\operatorname{gcd}(m, n)=1$, in the notation of (2.1). Suppose that $e_{1} e_{2}=m n$ for $\mathbf{e} \in \mathbb{Q}^{2}$ satisfying the above $p$-adic constraints. If $p \mid m n$ then we must have $\left\{v_{p}\left(e_{1}\right), v_{p}\left(e_{2}\right)\right\}=\{0,1\}$. Alternatively, if $p \nmid m n$, then $\left\{v_{p}\left(e_{1}\right), v_{p}\left(e_{2}\right)\right\}=\{0,0\}$ or $\{-1,1\}$. Given such e let us put $f_{i}$ to be the product of primes $p \nmid m n$ for which $v_{p}\left(e_{i}\right)=1$. Then we may assume that $e_{1}=e_{1}^{\prime} f_{1} / f_{2}$ and $e_{2}=e_{2}^{\prime} f_{2} / f_{1}$, where $e_{1}^{\prime}, e_{2}^{\prime}$ are square-free integers comprised of primes $p \mid m n$. On multiplying the first equation by $f_{2}^{2}$ and the second by $f_{1}^{2}$, and making a further change of variables, we see that it suffices to check local solubility with $\mathbf{e}=\left(e_{1}, e_{2}\right)$ replaced by $\mathbf{e}^{\prime \prime}=\left(e_{1}^{\prime} f, e_{2}^{\prime} f\right)$, where $f=f_{1} f_{2}$. In particular, we have the relation $e_{1}^{\prime \prime} e_{2}^{\prime \prime}=e_{1}^{\prime} e_{2}^{\prime} f^{2}=m n f^{2}$. We summarise our remarks in the following result.

Lemma 4.1. - Let $(a, b, c, d) \in S_{\mathrm{loc}}^{(\iota)}$. Then $X_{a, b, c, d}(\mathbb{Q}) \neq \emptyset$ if and only if there exists $\mathbf{e} \in \mathscr{B}^{2}$ and $f \in \mathscr{B}$, with $\operatorname{gcd}(f, m n)=1$ and

$$
e_{1} e_{2}=m n f^{2}
$$

such that the smooth variety

$$
\mathbb{A}^{6} \supset W_{\mathbf{e}}: \quad\left\{\begin{array}{l}
0 \neq Y_{1}^{2}+Z_{1}^{2}=e_{1} Q_{1}^{\prime}(U, V), \\
0 \neq Y_{2}^{2}+Z_{2}^{2}=e_{2} Q_{2}^{\prime}(U, V),
\end{array}\right.
$$

has solutions everywhere locally.

The thrust of Lemma 4.1 is that our task of determining elements of $S_{\text {glob }}^{(\iota)}$ in (2.4) has become a purely local problem. In fact, the varieties $W_{\mathbf{e}}$ are known to satisfy the Hasse principle and any $\mathbb{Q}$-point on $W_{\mathbf{e}}$ gives rise to a $\mathbb{Q}$-point on $X_{a, b, c, d}$. We shall henceforth refer to $W_{\mathbf{e}}$ as torsor equations. In reality, as explained in [4, $\left.\S \mathrm{VII}\right]$, any universal torsor over $X_{a, b, c, d}$ is birational to $\mathbb{P}^{1} \times C \times \mathbb{P}^{1} \times W_{\mathbf{e}}$, for a smooth conic $C$ defined over $\mathbb{Q}$. 
We proceed to study the sets $W_{\mathbf{e}}\left(\mathbb{Q}_{w}\right)$ for each $w \in \Omega$. When determining solubility over $\mathbb{Q}_{p}$ it will suffice to consider the existence of solutions $\left(y_{i}, z_{i}, u, v\right)$ with $u, v \in \mathbb{Z}_{p}$ coprime. Recall from $\$ 1$ our discussion of the Brauer group $\operatorname{Br}\left(X_{a, b, c, d}\right) / \operatorname{Br}(\mathbb{Q})$, which is generated by a single element, given by the quaternion algebra $\left(-1, a T^{2}+b\right)$. Given any local point $M_{w}=(y, z, u, v)$ in $X\left(\mathbb{Q}_{w}\right)$, we have

$$
\operatorname{inv}_{w}\left(\operatorname{ev}_{\mathscr{A}}\left(M_{w}\right)\right)= \begin{cases}0, & \text { if } Y^{2}+Z^{2}=Q_{1}(u, v) T^{2} \text { soluble over } \mathbb{Q}_{w}, \\ 1 / 2, & \text { otherwise. }\end{cases}
$$

According to our discussion of (1.8), in order for counter-examples to the Hasse principle to arise, we will need $\operatorname{inv}_{w}\left(\operatorname{ev}_{\mathscr{A}}\left(M_{w}\right)\right)$ to be locally constant for each $w \neq 2$, in which case we will show that there is a unique torsor $W_{\mathbf{e}}$ which has $\mathbb{Q}_{w}$-points.

4.1. Solubility over $\mathbb{Q}_{p}$ for odd $p$. - It will be convenient to retain our shorthand notation $Q_{i}^{\prime}$ for $Q_{i}^{\prime}(u, v)$ in what follows. The case in which $p$ is an odd prime not appearing in the factorisation of $e_{1} e_{2}$ is straightforward, as the following result shows.

Lemma 4.2. - Let $\mathbf{e} \in \mathscr{B}^{2}$ and let $p>2$, with $p \nmid e_{1} e_{2}$. Then $W_{\mathbf{e}}\left(\mathbb{Q}_{p}\right) \neq \emptyset$.

Proof. - If $p \equiv 1(\bmod 4)$ then the conclusion is trivial. Suppose now that $p \equiv 3(\bmod 4)$. The second paragraph in the proof of Lemma 3.1 shows that there exists $u, v \in \mathbb{Z}_{p}$ such that $v_{p}\left(Q_{1}^{\prime} Q_{2}^{\prime}\right)=0$. But then $v_{p}\left(e_{i} Q_{i}^{\prime}\right)=0$ for $i=1,2$, which concludes the proof.

Since $e_{1}, e_{2}$ are square-free and entirely composed of primes congruent to 3 modulo 4 , Lemma 4.2 completely handles the solubility question over $\mathbb{Q}_{p}$ for $p \equiv 1(\bmod 4)$. Turning to $p \equiv 3(\bmod 4)$, it remains to consider the possibility that $p \mid \operatorname{gcd}\left(m n, e_{1} e_{2}\right)$, together with the possibility that $p \nmid m n$ and $p \mid \mathbf{e}$. We may assume that

$$
\mathbf{e}=\left(e_{1}^{\prime} p^{k_{1}}, e_{2}^{\prime} p^{k_{2}}\right),
$$

with $\mathbf{k}=\left(k_{1}, k_{2}\right) \in\{0,1\}^{2}$ and $p \nmid e_{1}^{\prime} e_{2}^{\prime}$. Then the condition (4.1) demands that

$$
k_{1}+k_{2} \equiv v_{p}(m n)(\bmod 2) \text {. }
$$

In particular we have $\mathbf{k} \in\{(0,0),(1,1)\}$ when $v_{p}(m n)=0$ and $\mathbf{k} \in\{(0,1),(1,0)\}$ when $v_{p}(m n)=1$. Recall that $\Delta^{\prime}=a^{\prime} d^{\prime}-b^{\prime} c^{\prime}$. We proceed to establish the following result.

Lemma 4.3. - Let $(a, b, c, d) \in S_{\mathrm{loc}}^{(\iota)}$ and let $p \equiv 3(\bmod 4)$ with $k=v_{p}(m n) \in\{0,1\}$. Let $\mathbf{e}^{\prime} \in \mathscr{B}^{2}$ with $p \nmid e_{1}^{\prime} e_{2}^{\prime}$. Then one of the following must hold:

(i) if $\left[\frac{-a^{\prime} b^{\prime}}{p}\right]+\left[\frac{-c^{\prime} d^{\prime}}{p}\right] \leqslant 0$ then there exists a unique $\mathbf{k} \in\{0,1\}^{2}$ with $k_{1}+k_{2} \equiv k(\bmod 2)$ such that $W_{\left(e_{1}^{\prime} p^{k_{1}}, e_{2}^{\prime} p^{\left.k_{2}\right)}\right.}\left(\mathbb{Q}_{p}\right) \neq \emptyset$;

(ii) if $\left[\frac{-a^{\prime} b^{\prime}}{p}\right]=\left[\frac{-c^{\prime} d^{\prime}}{p}\right]=1$ and $k=0$, then $W_{\mathbf{e}^{\prime}}\left(\mathbb{Q}_{p}\right) \neq \emptyset$; furthermore, $W_{p \mathbf{e}^{\prime}}\left(\mathbb{Q}_{p}\right) \neq \emptyset$ if and only if $p \mid \Delta^{\prime}$;

(iii) if $\left[\frac{-a^{\prime} b^{\prime}}{p}\right]=\left[\frac{-c^{\prime} d^{\prime}}{p}\right]=1$ and $k=1$, then $W_{\left(e_{1}^{\prime} p, e_{2}^{\prime}\right)}\left(\mathbb{Q}_{p}\right) \neq \emptyset$ unless $v_{p}\left(a^{\prime}\right) \geqslant 2$ is even and $v_{p}\left(c^{\prime}\right)=1$, or $v_{p}\left(b^{\prime}\right) \geqslant 2$ is even and $v_{p}\left(d^{\prime}\right)=1$; likewise, $W_{\left(e_{1}^{\prime}, e_{2}^{\prime} p\right)}\left(\mathbb{Q}_{p}\right) \neq \emptyset$ unless $v_{p}\left(c^{\prime}\right) \geqslant 2$ is even and $v_{p}\left(a^{\prime}\right)=1$, or $v_{p}\left(d^{\prime}\right) \geqslant 2$ is even and $v_{p}\left(b^{\prime}\right)=1$.

In particular, whether or not $W_{\mathbf{e}}\left(\mathbb{Q}_{p}\right)$ is empty depends only on $v_{p}\left(e_{1}\right)$ and $v_{p}\left(e_{2}\right)$.

Remark 4.4. - Let $p$ be an odd prime. Lemmas 4.2 and 4.3 together show that whenever $X_{a, b, c, d}\left(\mathbb{Q}_{p}\right) \neq \emptyset$, there exists $\mathbf{k} \in\{0,1\}^{2}$, with $k_{1}+k_{2} \equiv v_{p}(m n)(\bmod 2)$, such that $W_{\left(p^{\left.k_{1}, p^{k_{2}}\right)}\right.}\left(\mathbb{Q}_{p}\right) \neq \emptyset$. 
Proof of Lemma 4.3. - Our arguments will have a similar flavour to the proof of Lemma 3.1. Beginning with case (i) we suppose without loss of generality that $\left[\frac{-c^{\prime} d^{\prime}}{p}\right]=-1$. Now any solution $u, v$ must have $2 \mid v_{p}\left(Q_{2}^{\prime}\right)$, whence the choice $\mathbf{k}=(1,1)$ (resp. $\left.\mathbf{k}=(0,1)\right)$ is not admissible when $k=0$ (resp. $k=1$ ). Finally we note that the case $\mathbf{k}=(0,0)$ is admissible when $k=0$ by Lemma 4.2, and the case $\mathbf{k}=(1,0)$ is admissible when $k=1$ since Lemma 3.1 implies that $\left[\frac{-a^{\prime} b^{\prime}}{p}\right]=1$.

Let us consider case (ii), wherein we have $k=0$ and $\left[\frac{-a^{\prime} b^{\prime}}{p}\right]=\left[\frac{-c^{\prime} d^{\prime}}{p}\right]=1$. Let us set

$$
\alpha=v_{p}\left(a^{\prime}\right), \quad \beta=v_{p}\left(b^{\prime}\right), \quad \gamma=v_{p}\left(c^{\prime}\right), \quad \delta=v_{p}\left(d^{\prime}\right), \quad \kappa=v_{p}\left(\Delta^{\prime}\right),
$$

with $\min \{\alpha, \gamma\}, \min \{\beta, \delta\} \leqslant 1$ and $\min \{\alpha, \beta\}=\min \{\gamma, \delta\}=0$. It is clear that $\mathbf{k}=(0,0)$ leads to a torsor with $p$-adic points, by Lemma 4.2. The choice $\mathbf{k}=(1,1)$ is permissible if and only if there exist coprime $u, v$ such that $v_{p}\left(Q_{i}^{\prime}\right) \equiv 1(\bmod 2)$ for $i=1,2$. If $\kappa=0$ then (2.7) implies that there are no such solutions. Alternatively, suppose that $\kappa \geqslant 1$. Suppose first that $\alpha=\beta=\gamma=\delta=0$. In particular we have $\left(\frac{-a^{\prime} b^{\prime}}{p}\right)=1$. If $\kappa \geqslant 2$ then we may choose $u, v$ such that $v_{p}\left(Q_{1}^{\prime}\right)=1$, in which case (2.7) implies that $v_{p}\left(Q_{2}^{\prime}\right)=1$. If $\kappa=1$ then we choose $u, v$ so that $v_{p}\left(Q_{1}^{\prime}\right)=3$ and it will follow that $v_{p}\left(Q_{2}^{\prime}\right)=1$. When $\max \{\alpha, \beta, \gamma, \delta\} \geqslant 1$ we argue according to parity. Without loss of generality we may suppose that $\beta=\delta=0$ and $\alpha, \gamma, \kappa \geqslant 1$. If $(\alpha, \gamma) \equiv(1,1)(\bmod 2)$ then it suffices to take $(u, v)=(1,0)$. If $\alpha, \gamma$ are of opposite parity, with say $(\alpha, \gamma) \equiv(0,1)(\bmod 2)$, we consider $(u, v)=\left(u^{\prime}, p^{\alpha / 2} v^{\prime}\right)$ chosen so that $v_{p}\left(Q_{1}^{\prime}\right) \equiv 1(\bmod 2)$. Then it follows that $v_{p}\left(Q_{2}^{\prime}\right) \equiv 1(\bmod 2)$ since $\alpha>\gamma$. Finally, the case $(\alpha, \gamma) \equiv(0,0)(\bmod 2)$ is impossible.

In case (iii) we must have $\mathbf{k}=(1,0)$ or $(0,1)$. By symmetry it will suffice to consider the case $\mathbf{k}=(1,0)$. We wish to determine when there exist coprime $u, v$ such that $v_{p}\left(Q_{1}^{\prime}\right)$ is odd and $v_{p}\left(Q_{2}^{\prime}\right)$ is even. Suppose first that $p \nmid a^{\prime} b^{\prime}$, so that $\left(\frac{-a^{\prime} b^{\prime}}{p}\right)=1$. Let $v_{p}\left(\Delta^{\prime}\right)=\kappa$. If $\kappa$ is even we may choose $u, v$ such that $v_{p}\left(Q_{1}^{\prime}\right)=\kappa+1$, in which case it follows from (2.7) that $v_{p}\left(Q_{2}^{\prime}\right)=\kappa$ is even. Likewise, if $\kappa$ is odd then $p \nmid c^{\prime} d^{\prime}$ and so we also have $\left(\frac{-c^{\prime} d^{\prime}}{p}\right)=1$. Thus we may choose $u, v$ such that $v_{p}\left(Q_{2}^{\prime}\right)=\kappa+1$ is even, in which case $v_{p}\left(Q_{1}^{\prime}\right)=\kappa$ is odd. We suppose now that $p \mid a^{\prime}$, say, with $a^{\prime}=p^{\alpha} a^{\prime \prime}$ and $p \nmid a^{\prime \prime} b^{\prime}$. If $p \mid d^{\prime}$, write $d^{\prime}=p^{\delta} d^{\prime \prime}$ with $p \nmid c^{\prime} d^{\prime \prime}$. If $\alpha$ is odd then we may take $(u, v)=(1,0)$. If $\alpha=2 \alpha^{\prime}$ is even then we choose $(u, v)=\left(u^{\prime}, p^{\alpha^{\prime}} v^{\prime}\right)$ such that $v_{p}\left(Q_{1}^{\prime}\right)=\alpha+1$, which is satisfactory. If, on the other hand, we write $c^{\prime}=p^{\gamma} c^{\prime \prime}$ for $\gamma \geqslant 0$ and $p \nmid c^{\prime \prime} d^{\prime}$, then the situation is more complicated. Suppose that $\alpha$ is odd and $\gamma$ is even. Then it suffices to take $(u, v)=(1,0)$. If $\alpha$ and $\gamma$ are both odd then $\alpha \neq \gamma$ by Lemma 3.1. Supposing that $\alpha=2 \alpha^{\prime}+1<\gamma$ then the argument used in the last part of the proof of Lemma 3.1 shows that $(u, v)=\left(1, p^{\alpha^{\prime}+1}\right)$ suffices. If $\alpha$ and $\gamma$ are both even then $\gamma=0$ and $\left(\frac{-a^{\prime \prime} b^{\prime}}{p}\right)=\left(\frac{-c^{\prime} d^{\prime}}{p}\right)=1$, resulting in a case that is easy to handle. Finally if $\alpha$ is even and $\gamma$ is odd, a case that requires $\alpha \geqslant 2$ and $\gamma=1$, then $v_{p}\left(\Delta^{\prime}\right)=1$ and it is impossible to find suitable $u, v$. The case in which $p \mid b^{\prime}$ is symmetric. This completes the proof of the lemma.

4.2. Solubility over $\mathbb{R}$. - We now turn to the set $W_{\mathbf{e}}(\mathbb{R})$, given $(a, b, c, d) \in S_{\text {loc }}^{(\iota)}$. Recall the notation for the sign function $\sigma(t) \in\{-,+\}$. We have $\sigma(a)=+$ and $\sigma(b, c, d) \neq(+,-,-)$, by part (i) of Lemma 3.1. On recalling that $m$ and $n$ are positive integers, we see that the constraint (4.1) demands that $e_{1}$ and $e_{2}$ share the same sign. Let $\Delta=a d-b c$. We wish to determine when there exist $u, v \in \mathbb{R}$ such that $\sigma\left(e_{i}\right) Q_{i}(u, v)>0$ for $i=1,2$, which will then ensure that $W_{\mathbf{e}}(\mathbb{R})$ is non-empty. We write $W_{ \pm}$for $W_{\mathbf{e}}(\mathbb{R})$ with $\sigma\left(e_{1}\right)=\sigma\left(e_{2}\right)= \pm$. 
Suppose first that $c>0$. Taking $u \neq 0$ and $v=0$ shows that $W_{+} \neq \emptyset$. If, furthermore, $b>0$ or $d>0$ then $W_{-}=\emptyset$. Finally, if $b, d<0$ then $W_{-} \neq \emptyset$, as can be seen by taking $u=0$ and $v \neq 0$. Next we consider the case $c<0$. Here, for $W_{+}$, we seek the existence of $u, v$ with $v \neq 0$ such that

$$
\frac{-d}{c}>\frac{u^{2}}{v^{2}}>\max \left\{\frac{-b}{a}, 0\right\} .
$$

This occurs if and only if $d>0$ and $\sigma(\Delta)=+$. For $W_{-}$we require instead the existence of $u, v$ with $v \neq 0$ such that

$$
\frac{-b}{a}>\frac{u^{2}}{v^{2}}>\max \left\{\frac{-d}{c}, 0\right\} .
$$

This occurs if and only if $b<0$ and $\sigma(\Delta)=-$. Finally we note that the case in which $\sigma(a)=\sigma(b)=+$ and $\sigma(c)=\sigma(d)=-$ does not enter into consideration. We summarise the situation in Table 1, in which the final column lists the possible signs of $e_{i}$ which give rise to a non-empty set $W_{\mathbf{e}}(\mathbb{R})$.

\begin{tabular}{|c|c|c|c|c|c|}
\hline & $\sigma(a)$ & $\sigma(b)$ & $\sigma(c)$ & $\sigma(d)$ & $\sigma\left(e_{i}\right): W_{\mathbf{e}}(\mathbb{R}) \neq \emptyset$ \\
\hline (i) & + & + & + & + & + \\
(ii) & + & - & + & - & + and - \\
(iii) & + & + & + & - & + \\
(iv) & + & - & + & + & + \\
(v) & + & + & - & + & + \\
(vi) & + & - & - & + & $\sigma(\Delta)$ \\
(vii) & + & - & - & - & - \\
\hline
\end{tabular}

TABLE $1 . W_{\mathbf{e}}(\mathbb{R})$ for $(a, b, c, d) \in S_{\text {loc }}$

4.3. Brauer group considerations. - Our work so far shows that for certain choices of coefficients $(a, b, c, d) \in S_{\text {loc }}^{(\iota)}$ there is more than one choice of torsor $W_{\mathbf{e}}$ with points everywhere locally. We need to show, using our discussion of the Brauer group above, that such $(a, b, c, d)$ actually belong to $S_{\text {glob }}^{(\iota)}$ and so are easily dealt with. In carrying out this plan let us write $j_{w}=\operatorname{inv}_{w}\left(\operatorname{ev}_{\mathscr{A}}\left(M_{w}\right)\right)$, for any local point $M_{w} \in X_{a, b, c, d}\left(\mathbb{Q}_{w}\right)$, whose value is given by (4.2). We may now record the following pair of results.

Lemma 4.5. - Let $(a, b, c, d) \in S_{\mathrm{loc}}^{(\iota)}$ arise in case (ii) of Table 1. Then $(a, b, c, d) \in S_{\mathrm{glob}}^{(\iota)}$.

Proof. - We argue by contradiction. Thus we may assume that $j_{\infty}$ is constant, else certainly $(a, b, c, d) \in S_{\text {glob }}^{(\iota)}$. But then if $j_{\infty}=0$ (resp. $j_{\infty}=1 / 2$ ) it follows from (4.2) that $\sigma\left(e_{1}\right)=+$ (resp. $\sigma\left(e_{1}\right)=-$ ). Hence case (ii) in Table 1 is impossible.

Lemma 4.6. - Let $(a, b, c, d) \in S_{\mathrm{loc}}^{(\iota)}$. If there is a prime $p \equiv 3(\bmod 4)$, for which there are precisely two choices of $\mathbf{k} \in\{0,1\}^{2}$ with $k_{1}+k_{2} \equiv v_{p}(m n)(\bmod 2)$ such that $W_{\left(p^{k_{1}}, p^{k_{2}}\right)}\left(\mathbb{Q}_{p}\right)$ is non-empty, then $(a, b, c, d) \in S_{\text {glob }}^{(\iota)}$. 
Proof. - We argue by contradiction. Thus we may assume that $j_{p}$ is constant, where $p$ is as in the statement of the lemma. If $j_{p}=0$ (resp. $j_{p}=1 / 2$ ), then (4.2) implies that $k_{1}=0$ (resp. $\left.k_{1}=1\right)$ and $k_{2}$ is uniquely determined from the congruence $k_{1}+k_{2} \equiv v_{p}(m n)(\bmod 2)$. This completes the proof of the lemma.

For the cases that are not covered by Lemmas 4.5 or 4.6, there exists a unique e such that for any valuation $w \in \Omega$, with $w \neq 2$, we have $W_{\mathbf{e}}\left(\mathbb{Q}_{w}\right) \neq \emptyset$. For this e it therefore remains to determine when $W_{\mathbf{e}}\left(\mathbb{Q}_{2}\right)$ is non-empty. This is so if and only if there exist coprime $u, v \in \mathbb{Z}_{2}$ such that $e_{i} Q_{i}^{\prime}(u, v) \in \mathscr{D}$ for $i=1,2$, where $\mathscr{D}$ is given by (2.9). This constraint depends only on e modulo 4 .

4.4. Solubility over $\mathbb{Q}_{2}$. - Recall the definition (2.9) of $\mathscr{D}$. In what follows it will be convenient to introduce the complementary set

$$
\overline{\mathscr{D}}=\left\{2^{n}(3+4 m): m \in \mathbb{Z}_{2}, n \in \mathbb{Z}\right\} .
$$

In this section we will be specifically interested in the set

$$
T_{\text {tot }}=\left\{t=(A, B, C, D) \in \mathbb{Z}^{4}: \begin{array}{ll}
2 \nmid A, \Upsilon=A D-B C \neq 0 \\
\\
\operatorname{gcd}(A, B)=\operatorname{gcd}(C, D)=1 \\
\operatorname{gcd}(B, D) \in \mathscr{A}
\end{array}\right\},
$$

where $\mathscr{A}$ is given by (2.1). Define

$$
R_{1}(U, V)=A U^{2}+B V^{2}, \quad R_{2}(U, V)=C U^{2}+D V^{2},
$$

We wish to classify exactly the $t \in T_{\text {tot }}$ for which there exist coprime $u, v \in \mathbb{Z}_{2}$ such that

(1) $R_{i}(u, v) \in \mathscr{D}$ for $i=1,2$; or

(2) $R_{1} R_{2}(u, v) \in \mathscr{D}$.

We see that case (2) holds if and only if there exist coprime $u, v \in \mathbb{Z}_{2}$ such that case (1) holds or such that $R_{i}(u, v) \in \bar{D}$ for $i=1,2$. Let us distinguish these two subsets of $T_{\text {tot }}$ by writing $T_{\text {tot }}^{(1)}$ and $T_{\text {tot }}^{(2)}$, respectively. In particular $T_{\text {tot }}^{(1)} \subset T_{\text {tot }}^{(2)}$.

Returning briefly to the question of solubility of $W_{\mathbf{e}}$ over $\mathbb{Q}_{2}$, suppose that

$$
e_{i} \equiv \epsilon_{i}(\bmod 4), \quad(i=1,2),
$$

for $\epsilon_{1}, \epsilon_{2} \in\{ \pm 1\}$. We will make the change of variables

$$
A=\epsilon_{1} a^{\prime}, \quad B=\epsilon_{1} b^{\prime}, \quad C=\epsilon_{2} c^{\prime}, \quad D=\epsilon_{2} d^{\prime} .
$$

In particular $(A, B, C, D) \in T_{\text {tot }}$ and we note that $e_{i} Q_{i}^{\prime}(u, v) \in \mathscr{D}$ if and only if $R_{i}(u, v) \in \mathscr{D}$, for $i=1,2$. From case (1) above we will be able to determine precisely when $W_{\mathbf{e}}\left(\mathbb{Q}_{2}\right) \neq \emptyset$. Likewise case (2) allows us to decide exactly when $X_{a, b, c, d}\left(\mathbb{Q}_{2}\right) \neq \emptyset$, as required for part (iv) of Lemma 3.1 .

It is now time to characterise the sets $T_{\text {tot }}^{(1)}$ and $T_{\text {tot }}^{(2)}$. Our argument differs according to the residue of $(A, B)$ modulo 4 . Consequently, given a pair $(i, j) \in(\mathbb{Z} / 4 \mathbb{Z})^{*} \times \mathbb{Z} / 4 \mathbb{Z}$, it will be convenient to put

$$
T_{\text {tot }}^{(k)}(i, j)=\left\{t \in T_{\text {tot }}^{(k)}:(A, B) \equiv(i, j)(\bmod 4)\right\},
$$

for $k \in\{1,2\}$. For each $(i, j)$, we shall list precise conditions which are both necessary and sufficient to ensure that $t$ belongs to $T_{\text {tot }}^{(1)}(i, j)$ or $T_{\text {tot }}^{(2)}(i, j)$. In doing so we will make repeated use of the observation that

$$
T_{\text {tot }}^{(2)}(i, j)=-T_{\text {tot }}^{(2)}(-i,-j) .
$$


Although we provide an explicit characterisation of all the sets $T_{\text {tot }}^{(1)}(i, j)$ and $T_{\text {tot }}^{(2)}(i, j)$, it is only the latter that will actually be used to calculate the numerical value of a certain 2-adic density in Lemma 6.2. Furthermore, we will lend support to the final numerical value obtained by a direct computer search. Consequently, the reader may choose to skip the proofs in this section at a first reading. As emphasised in $\$ 1$, however, once taken in conjunction with our work so far the results in this section give an explicit algorithm for testing whether or not any Châtelet surface $X_{a, b, c, d}$ has $\mathbb{Q}$-rational points. We will illustrate this aspect in 4.5 .

Suppose one is given coprime integers $r$, $s$. It will be useful to have a clear classification of precisely when there exists coprime $u, v \in \mathbb{Z}_{2}$ such that $r u^{2}+s v^{2} \in \mathscr{D}$, and what constraints are placed on any such solution, if any. Write $u=2^{\mu} u^{\prime}$ and $v=2^{\nu} v^{\prime}$, with $\min \{\mu, \nu\}=0$ and $2 \nmid u^{\prime} v^{\prime}$. It will be convenient to set

$$
u^{\prime}=1+2 u^{\prime \prime}, \quad v^{\prime}=1+2 v^{\prime \prime}, \quad \tilde{u}=u^{\prime \prime}+u^{\prime \prime 2}, \quad \tilde{v}=v^{\prime \prime}+v^{\prime \prime 2} .
$$

In particular $\tilde{u}, \tilde{v}$ are both even integers. Let us suppose that $r=2^{\varrho} r^{\prime}$ and $s=2^{\sigma} s^{\prime}$, with $\min \{\varrho, \sigma\}=0$ and $2 \nmid r^{\prime} s^{\prime}$. In Tables 2 and 3 we collect together conditions on the various parameters under which we have $r u^{2}+s v^{2} \in \mathscr{D}$.

\begin{tabular}{|l|l|l|}
\hline $\begin{array}{l}\text { condition } \\
\text { on } \nu\end{array}$ & $\begin{array}{l}\text { condition } \\
\text { on } r^{\prime}, s(\bmod 4)\end{array}$ & conditions on $u^{\prime}, v^{\prime}$ \\
\hline $2 \nu \leqslant \varrho-2$ & $s \equiv 1(\bmod 4)$ & \\
$2 \nu \geqslant \varrho+2$ & $r^{\prime} \equiv 1(\bmod 4)$ & \\
$2 \nu=\varrho+1$ & $r^{\prime} \equiv 3(\bmod 4)$ & \\
$2 \nu=\varrho-1$ & $s \equiv 3(\bmod 4)$ & \\
$2 \nu=\varrho$ & $r^{\prime}+s \equiv 2(\bmod 8)$ & \\
$2 \nu=\varrho$ & $r^{\prime}+s \equiv 4(\bmod 8)$ & $4\left(r^{\prime} \tilde{u}+s \tilde{v}\right) \equiv 4-r^{\prime}-s(\bmod 16)$ \\
$2 \nu=\varrho$ & $r^{\prime}+s \equiv 0(\bmod 8)$ & there exists $k \in \mathbb{Z}_{\geqslant 0}$ such that \\
& & $4\left(r^{\prime} \tilde{u}+s \tilde{v}\right) \equiv 2^{3+k}-r^{\prime}-s\left(\bmod 2^{5+k}\right)$ \\
\hline
\end{tabular}

TABLE 2. $r u^{2}+s v^{2} \in \mathscr{D}:$ the case $\varrho \geqslant 0$ and $\sigma=\mu=0$

We will establish Table 2 shortly. It represents an exhaustive list, so that in every case not covered in the table, one has $r u^{2}+s v^{2} \in \overline{\mathscr{D}}$. It is important to stress that in every case listed a choice of $u^{\prime}, v^{\prime}$ exists. It is only in the final two cases that additional constraints are imposed on $u^{\prime}, v^{\prime}$. In these cases one notes that the existence of suitable $k, \tilde{u}, \tilde{v}$ is automatic for the congruence class of $r^{\prime}+s(\bmod 8)$ considered. This then leads to the existence of suitable $u^{\prime}, v^{\prime}$ via (4.4), since for any $t \in 2 \mathbb{Z}_{2}$ and any $\ell \in \mathbb{N}$ one can always find $w \in \mathbb{Z} / 2^{\ell} \mathbb{Z}$ such that $w+w^{2} \equiv t\left(\bmod 2^{\ell}\right)$.

Proof of Table Q - For this we write $Q(U, V)=r U^{2}+s V^{2}$. Taking $\mu=0$ we note that

$$
Q\left(u^{\prime}, 2^{\nu} v^{\prime}\right)=2^{\min \{\varrho, 2 \nu\}}\left(2^{\varrho-\min \{\varrho, 2 \nu\}} r^{\prime} u^{\prime 2}+2^{2 \nu-\min \{\varrho, 2 \nu\}} s v^{\prime 2}\right) .
$$

When $\varrho \neq 2 \nu$ it is easy to characterise when $Q\left(u^{\prime}, 2^{\nu} v^{\prime}\right) \in \mathscr{D}$. Thus, if $\varrho>2 \nu$ then for $\varrho=2 \nu+1($ resp. $\varrho \geqslant 2 \nu+2)$ one requires $s \equiv 3(\bmod 4)($ resp. $s=1(\bmod 4))$. The case $\varrho<2 \nu$ is handled similarly. Suppose next that $\varrho=2 \nu$, so that

$$
Q\left(u^{\prime}, 2^{\nu} v^{\prime}\right)=2^{\varrho}\left(r^{\prime} u^{\prime 2}+s v^{\prime 2}\right) .
$$


Since $r^{\prime}$ and $s$ are odd we must have $r^{\prime}+s \in\{0,2,4,6\}(\bmod 8)$. The case $r^{\prime}+s \equiv 6(\bmod 8)$ is impossible since then $Q\left(u^{\prime}, 2^{\nu} v^{\prime}\right) \in \bar{D}$ for any odd $u^{\prime}, v^{\prime}$. If $r^{\prime}+s \equiv 2(\bmod 8)$, on the other hand, then $Q\left(u^{\prime}, 2^{\nu} v^{\prime}\right) \in \mathscr{D}$ for any odd $u^{\prime}, v^{\prime}$. For the remaining cases we note that

$$
2^{-\varrho} Q\left(u^{\prime}, 2^{\nu} v^{\prime}\right)=r^{\prime}+s+4\left(r^{\prime} \tilde{u}+s \tilde{v}\right),
$$

in the notation of (4.4). When $r^{\prime}+s \equiv 4(\bmod 8)$, this is clearly congruent to 4 modulo 8 since $\tilde{u}, \tilde{v}$ are both even. We conclude in this case that $Q\left(u^{\prime}, 2^{\nu} v^{\prime}\right) \in \mathscr{D}$ if and only if $u, v$ are chosen so that

$$
r^{\prime}+s+4\left(r^{\prime} \tilde{u}+s \tilde{v}\right) \equiv 4(\bmod 16),
$$

as claimed in the table. Similarly, if $r^{\prime}+s \equiv 0(\bmod 8)$, then $8 \mid 2^{-\varrho} Q\left(u^{\prime}, 2^{\nu} v^{\prime}\right)$ and we arrive at the constraint that there exists $k \in \mathbb{Z}_{\geqslant 0}$ such that

$$
r^{\prime}+s+4\left(r^{\prime} \tilde{u}+s \tilde{v}\right) \equiv 2^{3+k}\left(\bmod 2^{5+k}\right) .
$$

This concludes the proof of Table 2 .

We are now able to deduce a number of further tables from Table 2 , Table 3 follows by symmetry. We would also like to characterise precisely when there exist coprime $u, v \in \mathbb{Z}_{2}$ such that $r u^{2}+s v^{2} \in \overline{\mathscr{D}}$. Tables 4 and 5 are obtained from Tables 2 and 3 , respectively, by multiplying the right hand side of each congruence in the second column by -1 .

\begin{tabular}{|l|l|l|}
\hline $\begin{array}{l}\text { Condition } \\
\text { on } \mu\end{array}$ & condition & conditions on $u^{\prime}, v^{\prime}$ \\
\hline $2 \mu \leqslant \sigma-2$ & $r \equiv 1(\bmod 4)$ & \\
$2 \mu \geqslant \sigma+2$ & $s^{\prime} \equiv 1(\bmod 4)$ & \\
$2 \mu=\sigma+1$ & $s^{\prime} \equiv 3(\bmod 4)$ & \\
$2 \mu=\sigma-1$ & $r \equiv 3(\bmod 4)$ & \\
$2 \mu=\sigma$ & $r+s^{\prime} \equiv 2(\bmod 8)$ & \\
$2 \mu=\sigma$ & $r+s^{\prime} \equiv 4(\bmod 8)$ & $4\left(r \tilde{u}+s^{\prime} \tilde{v}\right) \equiv 4-r-s^{\prime}(\bmod 16)$ \\
$2 \mu=\sigma$ & $r+s^{\prime} \equiv 0(\bmod 8)$ & there exists $k \in \mathbb{Z}_{\geqslant 0}$ such that \\
& & $4\left(r \tilde{u}+s^{\prime} \tilde{v}\right) \equiv 2^{3+k}-r-s^{\prime}\left(\bmod 2^{5+k}\right)$ \\
\hline
\end{tabular}

TABLE 3. $r u^{2}+s v^{2} \in \mathscr{D}:$ the case $\sigma \geqslant 0$ and $\varrho=\nu=0$

\begin{tabular}{|l|l|l|}
\hline $\begin{array}{l}\text { condition } \\
\text { on } \nu\end{array}$ & $\begin{array}{l}\text { condition } \\
\text { on } r^{\prime}, s(\bmod 4)\end{array}$ & conditions on $u^{\prime}, v^{\prime}$ \\
\hline $2 \nu \leqslant \varrho-2$ & $s \equiv 3(\bmod 4)$ & \\
$2 \nu \geqslant \varrho+2$ & $r^{\prime} \equiv 3(\bmod 4)$ & \\
$2 \nu=\varrho+1$ & $r^{\prime} \equiv 1(\bmod 4)$ & \\
$2 \nu=\varrho-1$ & $s \equiv 1(\bmod 4)$ & \\
$2 \nu=\varrho$ & $r^{\prime}+s \equiv 6(\bmod 8)$ & \\
$2 \nu=\varrho$ & $r^{\prime}+s \equiv 4(\bmod 8)$ & $4\left(r^{\prime} \tilde{u}+s \tilde{v}\right) \equiv 4-r^{\prime}-s(\bmod 16)$ \\
$2 \nu=\varrho$ & $r^{\prime}+s \equiv 0(\bmod 8)$ & there exists $k \in \mathbb{Z}_{\geqslant 0} \operatorname{such} \operatorname{that}$ \\
& & $4\left(r^{\prime} \tilde{u}+s \tilde{v}\right) \equiv 2^{3+k}-r^{\prime}-s\left(\bmod 2^{5+k}\right)$ \\
\hline
\end{tabular}

TABLE 4. $r u^{2}+s v^{2} \in \overline{\mathscr{D}}$ : the case $\varrho \geqslant 0$ and $\sigma=\mu=0$ 


\begin{tabular}{|l|l|l|}
\hline $\begin{array}{l}\text { condition } \\
\text { on } \mu\end{array}$ & $\begin{array}{l}\text { condition } \\
\text { on } r, s^{\prime}(\bmod 4)\end{array}$ & conditions on $u^{\prime}, v^{\prime}$ \\
\hline $2 \mu \leqslant \sigma-2$ & $r \equiv 3(\bmod 4)$ & \\
$2 \mu \geqslant \sigma+2$ & $s^{\prime} \equiv 3(\bmod 4)$ & \\
$2 \mu=\sigma+1$ & $s^{\prime} \equiv 1(\bmod 4)$ & \\
$2 \mu=\sigma-1$ & $r \equiv 1(\bmod 4)$ & \\
$2 \mu=\sigma$ & $r+s^{\prime} \equiv 6(\bmod 8)$ & \\
$2 \mu=\sigma$ & $r+s^{\prime} \equiv 4(\bmod 8)$ & $4\left(r \tilde{u}+s^{\prime} \tilde{v}\right) \equiv 4-r-s^{\prime}(\bmod 16)$ \\
$2 \mu=\sigma$ & $r+s^{\prime} \equiv 0(\bmod 8)$ & there exists $k \in \mathbb{Z}_{\geqslant 0} \operatorname{such}$ that \\
& & $4\left(r \tilde{u}+s^{\prime} \tilde{v}\right) \equiv 2^{3+k}-r-s^{\prime}\left(\bmod 2^{5+k}\right)$ \\
\hline
\end{tabular}

TABLE 5. $r u^{2}+s v^{2} \in \overline{\mathscr{D}}:$ the case $\sigma \geqslant 0$ and $\varrho=\nu=0$

Remark 4.7. - Armed with Tables 25 we are able to record a rather succinct condition under which there exist odd coprime $u, v \in \mathbb{Z}_{2}$ such that $r u^{2}+s v^{2}$ belongs to $\mathscr{D}$ or $\overline{\mathscr{D}}$. Taking $\mu=\nu=0$ we see that there exist odd coprime $u, v \in \mathbb{Z}_{2}$ such that $r u^{2}+s v^{2} \in \mathscr{D}$ (resp. $\left.r u^{2}+s v^{2} \in \bar{D}\right)$ if and only if $r+s \in\{0,1,2,4,5\}(\bmod 8)($ resp. $r+s \in\{0,3,4,6,7\}(\bmod 8))$. Note that when $4 \mid r+s$, additional constraints are placed on the admissible $u, v$.

For the remainder of this section we will adhere to the notation

$$
B=2^{\beta} B^{\prime}, \quad C=2^{\gamma} C^{\prime}, \quad D=2^{\delta} D^{\prime},
$$

for integers $\beta, \gamma, \delta \geqslant 0$ such that $2 \nmid B^{\prime} C^{\prime} D^{\prime}$, with $\min \{\beta, \delta\} \leqslant 1$ and $\min \{\gamma, \delta\}=0$.

Lemma 4.8. - We have $t \in T_{\mathrm{tot}}^{(1)}(1,1)$ if and only if one of the following holds:

$-C \in \mathscr{D}$ or $D \in \mathscr{D}$;

$-(C, D) \equiv(3,3)(\bmod 4)$ with $A+B \equiv C+D \equiv 2(\bmod 8)$;

$-C \equiv 3(\bmod 4), \delta \geqslant 1$ and $D^{\prime} \equiv 3(\bmod 4)$, such that $C+D^{\prime} \equiv 2(\bmod 8)$ if $2 \mid \delta$;

$-D \equiv 3(\bmod 4), \gamma \geqslant 1$ and $C^{\prime} \equiv 3(\bmod 4)$, such that $C^{\prime}+D \equiv 2(\bmod 8)$ if $2 \mid \gamma$.

Moreover $t \in T_{\mathrm{tot}}^{(2)}(1,1)$ if and only if one of the following holds:

- $C \in \mathscr{D}$ or $D \in \mathscr{D}$;

$-(C, D) \equiv(3,3)(\bmod 4)$ with $A+B \equiv C+D(\bmod 8)$;

$-C \equiv 3(\bmod 4)$ and $D^{\prime} \equiv 3(\bmod 4)$, with one of the following:

- $2 \nmid \delta$,

- $2 \mid \delta, \delta \geqslant 2$ and $C+D^{\prime} \equiv 2(\bmod 8)$,

- $2 \mid \delta, \delta \geqslant 2$ and $A+B \equiv C+D^{\prime} \equiv 6(\bmod 8)$;

$-D \equiv 3(\bmod 4)$ and $C^{\prime} \equiv 3(\bmod 4)$, with one of the following:

- $2 \nmid \gamma$,

- $2 \mid \gamma, \gamma \geqslant 2$ and $C^{\prime}+D \equiv 2(\bmod 8)$,

- $2 \mid \gamma, \gamma \geqslant 2$ and $A+B \equiv C^{\prime}+D \equiv 6(\bmod 8)$.

Proof. - When $(A, B) \equiv(1,1)(\bmod 4)$, it is easy to see that $R_{1}(u, v) \in \mathscr{D}$ if and only if $\mu \geqslant 1$ or $\nu \geqslant 1$ or $\mu=\nu=0$ and $A+B \equiv 2(\bmod 8)$. Clearly $R_{2}(1,0) \in \mathscr{D}$ if $C \in \mathscr{D}$ and $R_{2}(0,1) \in \mathscr{D}$ if $D \in \mathscr{D}$. Suppose now that $C, D \in \overline{\mathscr{D}}$. If $(C, D) \equiv(3,3)(\bmod 4)$ then in order to have $t \in T_{\text {tot }}^{(1)}$ we must restrict to the case in which $u, v$ are odd. But then it follows from Remark 4.7 that $t \in T_{\text {tot }}^{(1)}(1,1)$ if and only if $A+B \equiv C+D \equiv 2(\bmod 8)$, with 
$t \in T_{\text {tot }}^{(2)}(1,1) \backslash T_{\text {tot }}^{(1)}(1,1)$ if and only if $A+B \equiv C+D \equiv 6(\bmod 8)$. Next we suppose that $\delta \geqslant 1$. If $A+B \equiv 6(\bmod 8)$ then we see that $t \in T_{\text {tot }}^{(2)}(1,1)$ by taking $(u, v)=(1,1)$. Next, Table 3 implies that $R_{2}(u, v) \in \mathscr{D}$ if and only if one of the following holds:

$-\nu=0,2 \mu=\delta+1$ and $D^{\prime} \equiv 3(\bmod 4)$,

$-\nu=0,2 \mu=\delta-1$ and $C \equiv 3(\bmod 4)$,

$-\nu=0,2 \mu=\delta$ and $C+D^{\prime} \equiv 2(\bmod 8)$.

Hence $t \in T_{\mathrm{tot}}^{(1)}(1,1)$ if and only if $\delta$ is odd or $\delta$ is even and $C+D^{\prime} \equiv 2(\bmod 8)$. Alternatively, if $\delta \geqslant 2$ is even then $t \in T_{\text {tot }}^{(2)}(1,1)$ if $C+D^{\prime} \equiv 6(\bmod 8)$ and $A+B \equiv 6(\bmod 8)$, by our existing argument. Finally, the case in which $D \equiv 3(\bmod 4)$ and $\gamma \geqslant 1$ is symmetric. The statement of the lemma now follows.

Lemma 4.9. - We have $t \in T_{\mathrm{tot}}^{(1)}(3,3)$ if and only if:

$-A+B \equiv 2(\bmod 8)$ and $C+D \in\{0,1,2,4,5\}(\bmod 8)$.

Moreover the characterisation of $T_{\mathrm{tot}}^{(2)}(3,3)$ follows from (4.3) and Lemma 4.8.

Proof. - In analysing $T_{\text {tot }}^{(1)}(3,3)$ we note $A+B \in\{2,6\}(\bmod 8)$. We must have $u, v$ both being odd in any solution and it therefore follows from Remark 4.7 that there exist odd $u, v$ such that $R_{i}(u, v) \in \mathscr{D}$, for $i=1,2$, if and only if $A+B \equiv 2(\bmod 8)$ and $C+D \in$ $\{0,1,2,4,5\}(\bmod 8)$.

Lemma 4.10. - Suppose that $t=(A, B, C, D) \equiv(1,3,3,1)(\bmod 4)$. Then $t \in T_{\text {tot }}^{(1)}$ if and only if one of the following holds:

$-A+B \equiv 0(\bmod 8)$;

$-A+B \equiv 4(\bmod 8)$ and $C+D \equiv 0(\bmod 8)$;

$-A+B \equiv 4(\bmod 8)$ and $A+B \equiv C+D(\bmod 16)$.

Moreover $t \notin T_{\text {tot }}^{(2)} \backslash T_{\text {tot }}^{(1)}$.

Proof. - Write

$$
A+B=2^{k_{1}+2} \ell_{1}, \quad C+D=2^{k_{2}+2} \ell_{2},
$$

for $2 \nmid \ell_{1} \ell_{2}$ and $k_{1}, k_{2} \geqslant 0$. We will show that $t \in T_{\text {tot }}^{(1)}$ if and only if $\left(k_{1}, k_{2}\right) \neq(0,0)$ or $\ell_{1}+\ell_{2} \not \equiv 0(\bmod 4)$. A little thought shows that this is equivalent to the constraints recorded in the first part of the lemma.

In determining whether or not each $R_{i}(u, v)$ belongs to $\mathscr{D}$ we must necessarily restrict to odd coprime values of $u, v \in \mathbb{Z}_{2}$. It will be convenient to set $r_{i}=R_{i} / 4$ for $i=1,2$ and $A D-B C=4 \Upsilon^{\prime}$. Recall the notation introduced in (4.4). We have the identities

$$
\begin{aligned}
D \ell_{1} 2^{k_{1}}-B \ell_{2} 2^{k_{2}} & =\Upsilon^{\prime}, \\
-C \ell_{1} 2^{k_{1}}+A \ell_{2} 2^{k_{2}} & =\Upsilon^{\prime},
\end{aligned}
$$

and

$$
\begin{aligned}
r_{1}(u, v) & =2^{k_{1}} \ell_{1}+A \tilde{u}+B \tilde{v}, \\
r_{2}(u, v) & =2^{k_{2}} \ell_{2}+C \tilde{u}+D \tilde{v}, \\
-C r_{1}(u, v)+A r_{2}(u, v) & =\Upsilon^{\prime}(1+4 \tilde{v}) .
\end{aligned}
$$

In what follows we will make frequent use of the fact that the map $\mathbb{Z}_{2} \rightarrow 2 \mathbb{Z}_{2}$, given by $w \mapsto w+w^{2}$, is surjective. 
By symmetry we may restrict attention to the case $k_{2} \geqslant k_{1}$. Assume that $k_{1} \geqslant 2$, with $k_{2} \geqslant k_{1}+1$. In this case we choose $\tilde{v}=0$ and $\tilde{u}=2^{k_{1}-1} \tilde{u}^{\prime}$, with $\tilde{u}^{\prime} \equiv 3(\bmod 4)$. In particular $\tilde{u}$ is even and we have

$$
r_{1} / 2^{k_{1}-1}=2 \ell_{1}+A \tilde{u}^{\prime} \equiv 1(\bmod 4), \quad r_{2} / 2^{k_{1}-1}=2^{k_{2}-k_{1}+1} \ell_{2}+C \tilde{u}^{\prime} \equiv 1(\bmod 4),
$$

as required. We may henceforth assume that $k_{1} \leqslant 1$ or $k_{2}=k_{1} \geqslant 2$.

Suppose that $k_{2}=k_{1}=k$ and $\ell_{1} \equiv \ell_{2}(\bmod 4)$. Either $\ell_{1}, \ell_{2}$ are congruent to 1 modulo 4 and we take $\tilde{u}=0$ and $\tilde{v}=2^{k+2}$, or $\ell_{1}, \ell_{2}$ are congruent to 3 modulo 4 and we take $\tilde{u}=2^{k+1}$ and $\tilde{v}=0$. Next suppose that $k_{2}=k_{1}=k \geqslant 1$ and $\ell_{1}+\ell_{2} \equiv 0(\bmod 4)$. In this case $k^{\prime}=v_{2}\left(\Upsilon^{\prime}\right) \geqslant k+2$. We choose $\tilde{v}=0$ and $\tilde{u}$ such that

$$
A \tilde{u} \equiv-2^{k} \ell_{1}+2^{k^{\prime}-1}\left(\bmod 2^{k^{\prime}+1}\right) .
$$

Then it follows that $r_{1} \equiv r_{2} \equiv 2^{k^{\prime}-1}\left(\bmod 2^{k^{\prime}+1}\right)$, as required. Next, when $k_{2}=k_{1}=0$ and $\ell_{1}+\ell_{2} \equiv 0(\bmod 4)$, it follows $r_{1}$ and $r_{2}$ are both odd, with $r_{1}+r_{2} \equiv 0(\bmod 4)$. In this case, therefore, we must have $t \notin T_{\text {tot }}^{(1)} \cup T_{\text {tot }}^{(2)}$.

Suppose now that $k_{1}=0$ and $k_{2} \geqslant 1$. Choose $\tilde{u}$ such that $2^{k_{2}} \ell_{2}+C \tilde{u} \equiv 2^{i}\left(\bmod 2^{2+i}\right)$ for $i \in\{1,2\}$, and $\tilde{v}=0$, so that $r_{2} \in \mathscr{D}$. Then

$$
r_{1}=\ell_{1}+A \tilde{u} \equiv \ell_{1}+A \bar{C}\left(2^{i}-2^{k_{2}} \ell_{2}\right)(\bmod 4) .
$$

If $k_{2} \geqslant 2$ we choose $i$ such that $\ell_{1} \equiv(-1)^{i}(\bmod 4)$. Alternatively, if $k_{2}=1$, we choose $i$ such that $\ell_{1} \equiv(-1)^{i-1}(\bmod 4)$. This then ensures that $r_{1} \in \mathscr{D}$, as required.

Let us now consider the case $k_{1}=1$, with $k_{2} \geqslant 2$, for which we take $\tilde{v}=0$. For any $i \in\{1,2\}$ we can choose $\tilde{u}$ such that $2^{k_{2}} \ell_{2}+C \tilde{u} \equiv 2^{1+i}\left(\bmod 2^{3+i}\right)$, which thereby implies that $r_{2} \in \mathscr{D}$ and $2 \mid \tilde{u}$. Next, with these choices for $\tilde{u}, \tilde{v}$, we have

$$
r_{1} / 2 \equiv \ell_{1}+A \bar{C}\left(2^{i}-2^{k_{2}-1} \ell_{2}\right)(\bmod 4) .
$$

Thus, in order to ensure that $r_{1} \in \mathscr{D}$, it suffices to take $i=2($ resp. $i=1)$ if $\ell_{1} \equiv 1(\bmod 4)$ and $k_{2} \geqslant 3$, or $\ell_{1} \equiv 3(\bmod 4)$ and $k_{2}=2\left(\operatorname{resp} . \ell_{1} \equiv 1(\bmod 4)\right.$ and $k_{2}=2$, or $\ell_{1} \equiv 3(\bmod 4)$ and $\left.k_{2} \geqslant 3\right)$.

Lemma 4.11. - We have $t \in T_{\mathrm{tot}}^{(1)}(1,3)$ if and only if one of the following holds:

$-C \in \mathscr{D}$

$-C \in \bar{D}$ and $C+D \in\{1,2,5\}(\bmod 8)$;

$-D \equiv 1(\bmod 4), \gamma=1$ and $C^{\prime} \equiv 3(\bmod 4)$;

$-D \equiv 3(\bmod 4), \gamma \geqslant 2$ and $C^{\prime} \equiv 3(\bmod 4)$, with $C^{\prime}+D \equiv 2(\bmod 8)$ if $2 \mid \gamma$;

$-(C, D) \equiv(3,1)(\bmod 4)$, with one of the following:

- $A+B \equiv 0(\bmod 8)$,

- $A+B \equiv 4(\bmod 8)$ and $C+D \equiv 0(\bmod 8)$,

- $A+B \equiv 4(\bmod 8)$ and $A+B \equiv C+D(\bmod 16)$.

Moreover $t \in T_{\mathrm{tot}}^{(2)}(1,3)$ if and only if one of the following holds:

$-C \in \mathscr{D}$;

$-C \in \overline{\mathscr{D}}, 2 \mid C$;

$-C \equiv 3(\bmod 4)$ and $C+D \in\{1,2,3,5,6,7\}(\bmod 8)$;

$-(C, D) \equiv(3,1)(\bmod 4)$, with one of the following:

- $A+B \equiv 0(\bmod 8)$,

- $A+B \equiv 4(\bmod 8)$ and $C+D \equiv 0(\bmod 8)$,

- $A+B \equiv 4(\bmod 8)$ and $A+B \equiv C+D(\bmod 16)$. 
Proof. - We first deal with $T_{\text {tot }}^{(1)}(1,3)$, the first two cases corresponding to the choices $(u, v)=$ $(1,0)$ or $(u, v) \equiv(1,1)(\bmod 2)$ such that $R_{1}(u, v) \in \mathscr{D}$, respectively. Assume now that $C \in \overline{\mathscr{D}}$ and $C+D \notin\{1,2,5\}(\bmod 8)$. Suppose that $\gamma=0$. If $D \equiv 1(\bmod 4)$ then the result follows from Lemma 4.10, If $D \equiv 2(\bmod 4)$ this case is already covered since then $C+D \equiv 1(\bmod 4)$. If $D \equiv 3(\bmod 4)$ then either $C+D \equiv 2(\bmod 8)$, which is already covered, or $C+D \equiv 6(\bmod 8)$ wherein $t \notin T_{\text {tot }}^{(1)}(1,3)$. The case $4 \mid D$ is also seen to be impossible. Suppose that $\gamma=1$. If $D \equiv 3(\bmod 4)$ then $C+D \in\{1,5\}(\bmod 8)$, a case that we have excluded. If $D \equiv 1(\bmod 4)$ then an analysis of Table 2 shows that $t \in T_{\text {tot }}^{(1)}(1,3)$ on taking $\mu=0$ and $\nu=1$. Suppose now that $\gamma \geqslant 2$. If $D \equiv 1(\bmod 4)$ then again $C+D \in\{1,5\}(\bmod 8)$. If $D \equiv 3(\bmod 4)$ then $\nu \geqslant 1$ and Table 2 easily leads to the constraints in the lemma.

For the characterisation of $T_{\text {tot }}^{(2)}(1,3)$, it will suffice to show that $t \in T_{\text {tot }}^{(2)}(1,3) \backslash T_{\text {tot }}^{(1)}(1,3)$ if and only if one of the following holds:

$-C \equiv 3(\bmod 4)$ and $C+D \equiv 6(\bmod 8)$;

$-D \equiv 3(\bmod 4), \gamma \geqslant 2$ is even and $C^{\prime} \equiv 3(\bmod 4)$, with $C^{\prime}+D \equiv 6(\bmod 8)$;

$-C \equiv 3(\bmod 4), \delta \geqslant 2$.

The first condition comes from Remark 4.7. Assume now that $C \in \overline{\mathscr{D}}$ and $C+D \in$ $\{0,3\}(\bmod 4)$. It remains to consider the case $(C, D)$ being congruent to $(3,0)$ or $(0,3)$ modulo 4 , since $t \notin T_{\text {tot }}^{(2)}(1,3) \backslash T_{\text {tot }}^{(1)}(1,3)$ when $(C, D) \equiv(3,1)(\bmod 4)$ by Lemma 4.10, In the latter case it is necessary to consider the case of even $\gamma \geqslant 2$ and $C^{\prime}+D \not \equiv 2(\bmod 8)$. One deduces from Table 4 that one must have $C^{\prime}+D \equiv 6(\bmod 8)$, as required. Turning to the case $(C, D) \equiv(3,0)(\bmod 4)$, for which one automatically has $t \notin T_{\text {tot }}^{(1)}(1,3)$, an analysis of Table 5 shows that $t \in T_{\mathrm{tot}}^{(2)}(1,3)$ precisely when $C+D^{\prime} \equiv 0(\bmod 4)$ if $D^{\prime} \equiv 1(\bmod 4)$ and $\delta$ is even.

Lemma 4.12. - We have $t \in T_{\mathrm{tot}}^{(1)}(1,0)$ if and only if one of the following holds:

$-C \in \mathscr{D}$ or $C+D \in\{0,1,2,4,5\}(\bmod 8)$, with $4 \nmid D$;

$-\gamma=1$ and $\left(C^{\prime}, D\right) \equiv(3,1)(\bmod 4)$;

$-\gamma \geqslant 2$ and $\left(C^{\prime}, D\right) \equiv(3,3)(\bmod 4)$, with $C^{\prime}+D \equiv 2(\bmod 8)$ if $2 \mid \gamma$.

Moreover $t \in T_{\mathrm{tot}}^{(2)}(1,0)$ if and only if one of the following holds:

$-C \in \mathscr{D}$ and $4 \nmid D$;

$-C \in \overline{\mathscr{D}}$ and $C+D \in\{0,1,2,4,5\}(\bmod 8)$;

$-C \in \overline{\mathscr{D}}, 4 \nmid D$ and $C+D \in\{3,6,7\}(\bmod 8)$, with one of the following:

- $C \equiv 3(\bmod 4)$ and $C+D \equiv 6(\bmod 8)$, with $A+B^{\prime} \equiv 6(\bmod 8)$ if $B^{\prime} \equiv 1(\bmod 4)$ and $2 \mid \beta$;

- $C^{\prime} \equiv 3(\bmod 4), \gamma=1$ and $D \equiv 1(\bmod 4)$;

- $C^{\prime} \equiv 3(\bmod 4), \gamma \geqslant 2$ and $D \equiv 3(\bmod 4)$, with one of the following:

$* 2 \nmid \gamma$;

$* 2 \mid \gamma$ and $C^{\prime}+D \equiv 2(\bmod 8)$;

* $2 \mid \gamma$ and $C^{\prime}+D \equiv 6(\bmod 8)$, with $A+B^{\prime} \equiv 6(\bmod 8)$ if $B^{\prime} \equiv 1(\bmod 4)$ and $2 \mid \beta$.

Proof. - Note that $\delta \leqslant 1$ and $\beta \geqslant 2$. Dealing first with the criteria for $t \in T_{\text {tot }}^{(1)}(1,0)$, the first condition arises from the consideration of solutions with $\nu=0$ and $\mu \geqslant 0$, using Remark 4.7. This case covers the case $\gamma=0$. Hence we may assume that $C \in \overline{\mathscr{D}}$. 
Suppose now that $\gamma \geqslant 1$. If $D \equiv 3(\bmod 4)$ then we must have $\nu \geqslant 1$ and it follows from Table 2 that $2 \nu=\gamma \pm 1$ or $2 \nu=\gamma$ with $C^{\prime}+D \equiv 2(\bmod 8)$. This easily leads to the overall constraint $C^{\prime}+D \equiv 2(\bmod 8)$ if $2 \mid \gamma$ and $D \equiv 3(\bmod 4)$. Next, the case $D \equiv 1(\bmod 4)$ and $B^{\prime} \equiv 1(\bmod 4)$ is handled by taking $(u, v)=(0,1)$. Finally, if $D \equiv 1(\bmod 4)$ and $B^{\prime} \equiv 3(\bmod 4)$ our argument differs according to the parity of $\beta$. If $2 \nmid \beta$ then Table 3 allows us to choose $2 \mu=\beta+1$. If $2 \mid \beta$ then we choose $2 \mu=2 \beta$ which yields the result, on eliminating the cases already handled by the constraint $C+D \in\{0,1,2,4,5\}(\bmod 8)$.

Having determined the criteria for $T_{\text {tot }}^{(1)}(1,0)$, we now turn to the corresponding criteria for $t \in T_{\text {tot }}^{(2)}(1,0)$. The first pair of alternatives come from the first part of the lemma. It remains to consider the case $C \in \overline{\mathscr{D}}, 4 \nmid D$ and $C+D \in\{3,6,7\}(\bmod 8)$. If $C \equiv 3(\bmod 4)$, so that $C+D \equiv 6(\bmod 8)$, then the desired constraints follow from a direct application of Table 4 . If $C^{\prime} \equiv 3(\bmod 4)$, with $\gamma \geqslant 1$, then we apply the first part of the lemma when $\gamma=1$. The treatment of the case $\gamma \geqslant 2$ follows from Tables 4 and 5 .

Lemma 4.13. - We have $t \in T_{\mathrm{tot}}^{(1)}(3,0)$ if and only if one of the following holds:

$-D \in \mathscr{D}$ and $4 \nmid D$, with $A+B^{\prime} \equiv 2(\bmod 8)$ if $2 \mid \beta$;

$-\delta=1$ and $D^{\prime} \equiv 3(\bmod 4)$, with $\beta \in\{2,3\}$.

Moreover the characterisation of $T_{\mathrm{tot}}^{(2)}(3,0)$ follows from (4.3) and Lemma 4.12.

Proof. - As in the previous lemma we note here that $\delta \leqslant 1$ and $\beta \geqslant 2$. In analysing $T_{\text {tot }}^{(1)}(3,0)$, it follows from Remark 4.7 that there are no solutions with $\mu=0$. We must therefore consider the existence of solutions with $\mu \geqslant 1$ and $\nu=0$. Calling upon Table 3 we must have one of the following:

$-2 \mu=\beta-1$,

$-2 \mu \geqslant \beta+2$ if $B^{\prime} \equiv 1(\bmod 4)$,

$-2 \mu=\beta+1$ if $B^{\prime} \equiv 3(\bmod 4)$,

$-2 \mu=\beta$ if $A+B^{\prime} \in\{0,2,4\}(\bmod 8)$,

in order to have $R_{1}(u, v) \in \mathscr{D}$. Moreover there are additional constraints on $u, v$ in the last case when $A+B^{\prime} \in\{0,4\}(\bmod 8)$. Suppose that $D \in \mathscr{D}$. Then either $B^{\prime} \equiv 1(\bmod 4)$, and we take $2 \mu \geqslant \beta+2$, or else $B^{\prime} \equiv 3(\bmod 4)$, and there exists a suitable choice of $\mu$ provided that $A+B^{\prime} \equiv 2(\bmod 8)$ when $\beta$ is even. The case $D \equiv 3(\bmod 4)$ being impossible, we suppose next that $\delta=1$ and $D^{\prime} \equiv 3(\bmod 4)$. Then Table 3 implies that we must have one of the following:

$-2 \mu=2$,

$-2 \mu=0$ if $C \equiv 3(\bmod 4)$,

in order to have $R_{2}(u, v) \in \mathscr{D}$. The lemma now easily follows.

Lemma 4.14. - We have $t \in T_{\mathrm{tot}}^{(1)}(1,2)$ if and only if one of the following holds:

$-C \in \mathscr{D}$

$-C^{\prime} \equiv 3(\bmod 4)$, with one of the following:

- $D \equiv 1(\bmod 4), \gamma \geqslant 1$,

- $D \equiv 3(\bmod 4), \gamma \geqslant 1$ and $C^{\prime}+D \equiv 2(\bmod 8)$ if $2 \mid \gamma$,

- $B^{\prime} \equiv 3(\bmod 4), \gamma=0, \delta \in\{0,2,3\}$ and $D^{\prime} \equiv 1(\bmod 4)$,

- $B^{\prime} \equiv 3(\bmod 4), \gamma=0, \delta \in\{1,2,3\}$ and $D^{\prime} \equiv 3(\bmod 4)$, with $C+D^{\prime} \equiv 2(\bmod 8)$ if $\delta=2$,

- $B^{\prime} \equiv 1(\bmod 4), \gamma=0$ and $D^{\prime} \equiv 1(\bmod 4)$, 
- $B^{\prime} \equiv 1(\bmod 4), \gamma=0, \delta \geqslant 3$ and $D^{\prime} \equiv 3(\bmod 4)$, with $C+D^{\prime} \equiv 2(\bmod 8)$ if $2 \mid \delta$. Moreover $t \in T_{\mathrm{tot}}^{(2)}(1,2)$ if and only if one of the following holds:

$-C \in \mathscr{D}$;

$-C^{\prime} \equiv 3(\bmod 4), \gamma \geqslant 1$

$-C \equiv 3(\bmod 4), \gamma=0$, with one of the following:

- $B^{\prime} \equiv D^{\prime}(\bmod 4)$,

- $\left(B^{\prime}, D^{\prime}\right) \in\{(3,1),(1,3)\}(\bmod 4), \delta \neq 1$.

Proof. - In this setting $\beta=1$. Beginning with $T_{\text {tot }}^{(1)}(1,2)$, the first constraint follows on making the choice $(u, v)=(1,0)$. Thus we may suppose that $c \in \overline{\mathscr{D}}$. An analysis of Table 2 easily allows one to handle the solutions in which $\nu \geqslant 1$ and $\mu=0$. In this case we have $A u^{2}+2 B^{\prime} v^{2} \equiv 1(\bmod 4)$ and $2^{\gamma} C^{\prime} u^{2}+D v^{2} \in \mathscr{D}$ if and only if one of the following holds:

$-2 \nu=\gamma+1$,

$-2 \nu \leqslant \gamma-2$ if $D \equiv 1(\bmod 4)$,

$-2 \nu=\gamma-1$ if $D \equiv 3(\bmod 4)$,

$-2 \nu=\gamma$ if $C^{\prime}+D \in\{0,2,4\}(\bmod 8)$.

In particular it suffices to have $\gamma \geqslant 1$ when $D \equiv 1(\bmod 4)$. When $D \equiv 3(\bmod 4)$ then any odd $\gamma \geqslant 1$ is satisfactory and even $\gamma \geqslant 2$ is satisfactory only when $C^{\prime}+D \equiv 2(\bmod 8)$.

We now consider possible solutions with $\nu=0$, using Table 3. The case $\mu=0$ being impossible, we pass to the case $\mu=1$, in which case we must have $B^{\prime} \equiv 3(\bmod 4)$ and $\gamma=0$, the case $\gamma \geqslant 1$ already having been handled. It follows that $2=\delta-1$ or $2 \geqslant \delta+2$ or $2=\delta$ when $D^{\prime} \equiv 1(\bmod 4)$. Furthermore, if $D^{\prime} \equiv 3(\bmod 4)$ then the table implies that $2=\delta-1$ or $2=\delta+1$ or $2=\delta$, with the last only taking place if $C+D^{\prime} \equiv 2(\bmod 8)$. Let us now turn to solutions with $\mu \geqslant 2$ and $\gamma=0$, so that $B^{\prime} \equiv 1(\bmod 4)$. We deduce from Table 3 that it suffices to take $\mu$ so that $2 \mu \geqslant \delta+2$ when $D^{\prime} \equiv 1(\bmod 4)$. When $D^{\prime} \equiv 3(\bmod 4)$ then the table implies that $2 \mu=\delta-1$ or $2 \mu=\delta+1$ or $2 \mu=\delta$, with the last only taking place if $C+D^{\prime} \equiv 2(\bmod 8)$. This easily leads to the remaining constraints in the statement of the lemma.

We now determine $T_{\text {tot }}^{(2)}(1,2)$, for which it suffices to show that $t \in T_{\text {tot }}^{(2)}(1,2) \backslash T_{\text {tot }}^{(1)}(1,2)$ if and only if $C \in \overline{\mathscr{D}}$, with one of the following holding:

$-D \equiv 3(\bmod 4), \gamma \geqslant 2$ is even and $C^{\prime}+D \equiv 6(\bmod 8)$;

$-B^{\prime} \equiv 3(\bmod 4), \gamma=0, D^{\prime} \equiv 1(\bmod 4)$ and $\delta \geqslant 4$;

$-B^{\prime} \equiv 3(\bmod 4), \gamma=0, D^{\prime} \equiv 3(\bmod 4)$, with one of the following:

- $\delta \notin\{1,2,3\}$,

- $\delta=2$ and $C+D^{\prime} \equiv 6(\bmod 8)$;

$-B^{\prime} \equiv 1(\bmod 4), D^{\prime} \equiv 3(\bmod 4), \gamma=\delta=0$;

$-B^{\prime} \equiv 1(\bmod 4), D^{\prime} \equiv 3(\bmod 4), \gamma=0, \delta \geqslant 2$ even with $C+D^{\prime} \equiv 6(\bmod 8)$.

To see this we must have $\nu=0$ in any solution, else $R_{1}(u, v) \in \mathscr{D}$. Remark 4.7 shows that we have solutions with $R_{i}(u, v) \in \overline{\mathscr{D}}$ for $i=1,2$, with $\mu=\nu=0$ if and only if $C+D \in\{0,3,4,6,7\}(\bmod 8)$ since $A+B \in\{3,7\}(\bmod 8)$. If $\gamma \geqslant 1$ is even and $D \equiv 3(\bmod 4)$ then it follows that $t \in T_{\text {tot }}^{(2)}(1,2) \backslash T_{\text {tot }}^{(1)}(1,2)$ if $C^{\prime}+D \equiv 6(\bmod 8)$. Turning to the case $\gamma=0$, as soon as $\delta \geqslant 3$ it is possible to find $u, v$ such that $R_{i}(u, v) \in \overline{\mathscr{D}}$ for $i=1,2$, since then $C+D \in\{3,7\}(\bmod 8)$. Suppose that $B^{\prime} \equiv 3(\bmod 4)$. If $D^{\prime} \equiv 1(\bmod 4)$ and $\delta=1$ then we do not get points in $T_{\text {tot }}^{(2)}(1,2) \backslash T_{\text {tot }}^{(1)}(1,2)$. If $D^{\prime} \equiv 3(\bmod 4)$ and $\delta \in\{0,2\}$ then we do get points in $T_{\text {tot }}^{(2)}(1,2) \backslash T_{\text {tot }}^{(1)}(1,2)$ by taking $\mu \geqslant 2$. It remains to consider the case $B^{\prime} \equiv 1(\bmod 4)$ and 
$D^{\prime} \equiv 3(\bmod 4)$, for which we must have $\mu \in\{0,1\}$, together with $2 \mu \leqslant \delta-2$ or $2 \mu \geqslant \delta+2$ or $2 \mu=\delta$, the latter only holding when $C+D^{\prime} \equiv 6(\bmod 8)$. Taking $\mu=1$ we see that $\delta=0$ or $\delta \geqslant 4$ is permissible, with $\delta=2$ being permissible if $C+D^{\prime} \equiv 6(\bmod 8)$.

Lemma 4.15. - We have $t \in T_{\mathrm{tot}}^{(1)}(3,2)$ if and only if one of the following holds:

$-C+D \in\{0,1,2,4,5\}(\bmod 8)$;

$-D \equiv 1(\bmod 4)$ and $C+D \in\{3,6\}(\bmod 8)$;

$-C+D \equiv 3(\bmod 8), \delta=1$ and $D^{\prime} \equiv B^{\prime}(\bmod 4)$;

$-C \equiv 3(\bmod 4), \delta \geqslant 2$, with one of the following:

- $B^{\prime} \equiv 3(\bmod 4), \delta=3$,

- $B^{\prime} \equiv 3(\bmod 4), \delta=2$ and $C+D^{\prime} \in\{0,2,4\}(\bmod 8)$,

- $\left(B^{\prime}, D^{\prime}\right) \equiv(1,1)(\bmod 4)$,

- $\left(B^{\prime}, D^{\prime}\right) \equiv(1,3)(\bmod 4), \delta \geqslant 3$ and $C+D^{\prime} \equiv 2(\bmod 8)$ if $2 \mid \delta$.

Moreover the characterisation of $T_{\mathrm{tot}}^{(2)}(3,2)$ follows from (4.3) and Lemma 4.14.

Proof. - In this setting $\beta=1$ and we need only deal with the criteria for $T_{\text {tot }}^{(1)}(3,2)$. We note that $\nu=0$ in any solution. Suppose first that $\mu=\nu=0$. The first constraint then follows immediately from Remark 4.7. If $C+D \equiv 6(\bmod 8)$ then it is clear that the case $(C, D) \equiv(3,3)(\bmod 4)$ doesn't produce a point in $T_{\text {tot }}^{(1)}(3,2)$. If $(C, D) \equiv(1,1)(\bmod 4)$ then we choose $(u, v)=\left(2 u^{\prime}, 1\right)$, with $u^{\prime}$ such that $R_{1}(u, v) \in \mathscr{D}$. It therefore remains to consider the case $C+D \equiv 3(\bmod 4)$, in which we must have $D \not \equiv 3(\bmod 4)$. When $D \equiv 1(\bmod 4)$ it suffices to take $u=0($ resp. $u=2)$ if $B^{\prime} \equiv 1(\bmod 4)\left(\operatorname{resp} . B^{\prime} \equiv 3(\bmod 4)\right)$. Suppose that $D=2 D^{\prime}$ with $2 \nmid D^{\prime}$. If $D^{\prime} \equiv B^{\prime} \equiv 1(\bmod 4)$ then one takes $(u, v)=(0,1)$, while if $D^{\prime} \equiv B^{\prime} \equiv 3(\bmod 4)$ then one takes $(u, v)=(2,1)$. Table 3 implies that $t \notin T_{\text {tot }}^{(1)}(3,1)$ if $D^{\prime} \not \equiv B^{\prime}(\bmod 4)$.

Now suppose that $C \equiv 3(\bmod 4)$ and $\delta \geqslant 2$. Then $B^{\prime}$ is either congruent to 1 or 3 modulo 4 and we proceed to consider the latter case first. Since $A=B^{\prime} \equiv 3(\bmod 4)$ it follows from Table 3 that $2 \mu \in\{0,2\}$, whence in fact $\mu=1$ and $\delta \leqslant 3$. The case $\delta=3$ presents no problems. If $\delta=2$ then we require odd coprime $u^{\prime}, v \in \mathbb{Z}_{2}$ such that $C u^{\prime 2}+D^{\prime} v^{2} \in \mathscr{D}$, which is always possible if $C+D^{\prime} \not \equiv 6(\bmod 8)$. Now suppose that $B^{\prime} \equiv 1(\bmod 4)$. According to Table 3 we must have $\mu \geqslant 2$. The case $D^{\prime} \equiv 1(\bmod 4)$ is resolved on taking $(u, v)=(0,1)$. If $D^{\prime} \equiv 3(\bmod 4)$ then we must have $2 \mu \in\{\delta-1, \delta+1\}$, or else $2 \mu=\delta$ if $C+D^{\prime} \equiv 2(\bmod 8)$. Thus if $\delta \geqslant 4$ is even we take $2 \mu=\delta$ and if $\delta \geqslant 3$ is odd we take $2 \mu=\delta+1$.

4.5. A worked example. - In order to illustrate our investigation so far, it is instructive to verify Example 5.4 in [3, §5], concerning the family of Châtelet surfaces $X=X_{1,1-k,-1, k}$ for $k \in \mathbb{Z}$ such that $k \notin\{0,1\}$. In this setting $\Delta=1$ and $m=n=1$, in the notation of $\oint_{2}$, We wish to show that there exists $w \in \Omega$ such that $X\left(\mathbb{Q}_{w}\right)=\emptyset$ if and only if

$$
k<0 \text { or } k=4^{n}(8 m+7) \text { for } n \geqslant 2, m \geqslant 0 .
$$

We will also show that $X(\mathbb{Q})=\emptyset$ if and only if

$$
k<0 \text { or } k \equiv 3(\bmod 4) \text { or } k=4^{n}(8 m+7) \text { for } n \geqslant 1, m \geqslant 0 .
$$

This recovers [3, Prop. C], since it shows that for each positive integer $k \equiv 3(\bmod 4)$, the surface $X=X_{1,1-k,-1, k}$ fails the Hasse principle.

According to Lemma 3.1 we have $X(\mathbb{R})=\emptyset$ if and only $k<0$. Since $X\left(\mathbb{Q}_{p}\right) \neq \emptyset$ for any odd prime, it remains to determine when $X\left(\mathbb{Q}_{2}\right)=\emptyset$, to complete the description in (4.5). 
Turning to (4.6), for which we suppose that $k>0$ and $k \neq 4^{n}(8 m+7)$ for $n \geqslant 2, m \geqslant 0$, it follows from Lemma 4.1 that $X(\mathbb{Q}) \neq \emptyset$ if and only if there exists $f \in \mathscr{B}$ and $\epsilon \in\{ \pm 1\}$ such that $W_{(\epsilon f, \epsilon f)}$ has solutions in $\mathbb{Q}_{w}$ for every $w \in \Omega$. In fact we will be forced to take $\epsilon=f=1$. It follows from part (vi) of Table 1 that $W_{(\epsilon f, \epsilon f)}(\mathbb{R}) \neq \emptyset$ if we take $\epsilon=1$. Lemmas 4.2 and 4.3 show that $W_{(f, f)}\left(\mathbb{Q}_{p}\right) \neq \emptyset$ for every odd prime $p$, provided that $f=1$, which we now assume. We thereby retrieve $\left[3\right.$, Lemme 5.4.1], which states that $X(\mathbb{Q}) \neq \emptyset$ if and only if $W\left(\mathbb{Q}_{2}\right) \neq \emptyset$, with $W=W_{(1,1)}$.

Our final task is to determine precisely when $X\left(\mathbb{Q}_{2}\right)$ and $W\left(\mathbb{Q}_{2}\right)$ are empty. Let us set $(a, b, c, d)=(1,1-k,-1, k)$. If $k \in\{1,2\}(\bmod 4)$, so that $b \in\{0,3\}(\bmod 4)$, then it follows from Lemmas 4.11 and 4.12 that $(a, b, c, d) \in T_{\text {tot }}^{(1)}$, whence $X\left(\mathbb{Q}_{2}\right)$ and $W\left(\mathbb{Q}_{2}\right)$ are both nonempty. Suppose next that $k \equiv 3(\bmod 4)$, so that $b \equiv 2(\bmod 4)$ and we turn to Lemma 4.14. The first part of this result ensures that $W\left(\mathbb{Q}_{2}\right)=\emptyset$, whereas the second part implies that $X\left(\mathbb{Q}_{2}\right) \neq \emptyset$. Finally we must consider the case $k=2^{n} k^{\prime}$, with $n \geqslant 2$ and $2 \nmid k^{\prime}$. In particular $(a, b) \equiv(1,1)(\bmod 4)$ and we apply Lemma 4.8. This readily implies that $(a, b, c, d) \in T_{\text {tot }}^{(1)}$ if and only if $k^{\prime} \not \equiv 7(\bmod 8)$, whenever $n$ is even. Likewise, $(a, b, c, d) \in T_{\text {tot }}^{(2)}$ if and only if $k^{\prime} \not \equiv 7(\bmod 8)$, whenever $n$ is even and $n \geqslant 4$.

Bringing everything together, we conclude that $X\left(\mathbb{Q}_{2}\right)=\emptyset$ if and only if $k=4^{n}(8 m+7)$ for $n \geqslant 2, m \geqslant 0$, whereas $W\left(\mathbb{Q}_{2}\right)=\emptyset$ if and only if $k \equiv 3(\bmod 4)$ or $k=4^{n}(8 m+7)$ for $n \geqslant 1, m \geqslant 0$. This completes the necessity and sufficiency of the conditions (4.5) and (4.6).

\section{Asymptotics: preliminaries}

The purpose of this section is to set the scene for the final analysis of the counting functions $N_{\text {loc }}(P)$ and $N_{\mathrm{Br}}(P)$, defined in (1.6). According to our work in 92 , it is clear that

$$
\begin{aligned}
& N_{\mathrm{loc}}(P)=\frac{1}{4} \sum_{\iota \in\{0,1\}} \#\left\{(a, b, c, d) \in S_{\mathrm{loc}}^{(\iota)}: \max \{|a|,|b|,|c|,|d|\} \leqslant P\right\}, \\
& N_{\mathrm{Br}}(P)=\frac{1}{4} \sum_{\iota \in\{0,1\}} \#\left\{(a, b, c, d) \in S_{\mathrm{loc}}^{(\iota)}: \begin{array}{l}
\max \{|a|,|b|,|c|,|d|\} \leqslant P \\
X_{a, b, c, d}(\mathbb{Q})=\emptyset
\end{array}\right\},
\end{aligned}
$$

where $S_{\text {loc }}^{(\iota)}$ is given by (2.4). Furthermore, Theorem 1.2 will require us to establish an asymptotic formula for

$$
N(P)=\frac{1}{4} \#\left\{(a, b, c, d) \in S_{\mathrm{tot}}: \max \{|a|,|b|,|c|,|d|\} \leqslant P\right\},
$$

where $S_{\text {tot }}$ is given by (2.2). This is achieved in Lemma 5.2, the proof of which will serve as a warm-up for the treatments of $N_{\text {loc }}(P)$ and $N_{\mathrm{Br}}(P)$, in $\$ 6$ and $\$ 7$ respectively.

For given non-zero integers $a, d$ with modulus at most $P$, it follows from the trivial estimate for the divisor function that there are $O\left(P^{\varepsilon}\right)$ choices for integers $b, c$ such that $a d-b c=0$. At the expense of adding an error term $O\left(P^{2+\varepsilon}\right)$, this shows that we can henceforth drop the constraint $a d-b c \neq 0$ from the definitions (2.2), (2.3) of $S_{\text {tot }}$ and $S_{\text {tot }}^{(\iota)}$, respectively.

Before proceeding to our assessment of (5.1) $-(5.3)$, we first set out a basic estimate that will be useful to us, in which we recall the definition (2.10) of $\varphi_{1}^{*}=\varphi^{*}$. 
Lemma 5.1. - Let $a \in \mathbb{Z}$ and $q_{1}, q_{2} \in \mathbb{N}$ be such that $\operatorname{gcd}\left(q_{1}, q_{2}\right)=1$. Let $\varepsilon>0$ and $x, T \geqslant 1$, with $x \gg T q_{2} q_{1}^{\varepsilon}$. Then we have

$$
\#\left\{n \leqslant x: \begin{array}{l}
\operatorname{gcd}\left(n, q_{1}\right)=1 \\
n \equiv a\left(\bmod q_{2}\right)
\end{array}\right\}=\frac{\varphi^{*}\left(q_{1}\right) x}{q_{2}}\left(1+O\left(\frac{1}{T^{1-\varepsilon}}\right)\right) .
$$

Proof. - This follows on noting that the left hand side is $\varphi^{*}\left(q_{1}\right) x / q_{2}+O\left(2^{\omega\left(q_{1}\right)}\right)$.

Recall the definitions (2.1) of $\mathscr{A}$ and $\mathscr{B}$. Given any positive integers $N_{1}, N_{2}$ we write $d \mid N_{2}^{\infty}$ if any prime divisor of $d$ is also a prime divisor of $N_{2}$, and we write $\operatorname{gcd}\left(N_{1}, N_{2}^{\infty}\right)$ for the largest such divisor which is also a divisor or $N_{1}$. It is now time to make a number of changes of variables in order to simplify the various conditions that arise in the sets $S_{\text {tot }}$ and $S_{\text {loc }}^{(\iota)}$. In the former set we will always choose representative coordinates in such a way that $a>0$. Let $(a, b, c, d) \in S_{\text {tot }}$. We write

$$
m=\operatorname{gcd}(a, b), \quad n=\operatorname{gcd}(c, d),
$$

so that $m, n \in \mathscr{B}$ and $\operatorname{gcd}(m, n)=1$. We make the initial change of variables

$$
a=m a^{\prime}, \quad b=\varepsilon_{2} m b^{\prime}, \quad c=\varepsilon_{3} n c^{\prime}, \quad d=\varepsilon_{4} n d^{\prime},
$$

where $\left(\varepsilon_{2}, \varepsilon_{3}, \varepsilon_{4}\right)=\sigma(b, c, d)$ and $a^{\prime}, b^{\prime}, c^{\prime}, d^{\prime} \in \mathbb{N}$ satisfy $\operatorname{gcd}\left(a^{\prime}, b^{\prime}\right)=\operatorname{gcd}\left(c^{\prime}, d^{\prime}\right)=1$. Note that this is a departure from the change of variables used in $\oint_{2}$, where we allowed $a^{\prime}, b^{\prime}, c^{\prime}, d^{\prime}$ to have arbitrary sign. Next, we define

$$
\begin{array}{ll}
\ell_{1}=\operatorname{gcd}\left(a^{\prime},(m n)^{\infty}\right), & \ell_{2}=\operatorname{gcd}\left(b^{\prime},(m n)^{\infty}\right), \\
\ell_{3}=\operatorname{gcd}\left(c^{\prime},(m n)^{\infty}\right), & \ell_{4}=\operatorname{gcd}\left(d^{\prime},(m n)^{\infty}\right) .
\end{array}
$$

We now make the further change of variables

$$
a^{\prime}=\ell_{1} a^{\prime \prime}, \quad b^{\prime}=\ell_{2} b^{\prime \prime}, \quad c^{\prime}=\ell_{3} c^{\prime \prime}, \quad d^{\prime}=\ell_{4} d^{\prime \prime},
$$

for $a^{\prime \prime}, b^{\prime \prime}, c^{\prime \prime}, d^{\prime \prime} \in \mathbb{N}$ satisfying $\operatorname{gcd}\left(a^{\prime \prime} b^{\prime \prime} c^{\prime \prime} d^{\prime \prime}, m n\right)=1$. We have $\operatorname{gcd}\left(a^{\prime}, b^{\prime}\right)=\operatorname{gcd}\left(c^{\prime}, d^{\prime}\right)=1$ if and only if $\operatorname{gcd}\left(a^{\prime \prime}, b^{\prime \prime}\right)=\operatorname{gcd}\left(c^{\prime \prime}, d^{\prime \prime}\right)=1$ and

$$
\operatorname{gcd}\left(\ell_{1}, \ell_{2}\right)=\operatorname{gcd}\left(\ell_{3}, \ell_{4}\right)=1 \text {. }
$$

Likewise, we see that $\operatorname{gcd}(a, c), \operatorname{gcd}(b, d) \in \mathscr{A}$ if and only if $\operatorname{gcd}\left(a^{\prime \prime}, c^{\prime \prime}\right), \operatorname{gcd}\left(b^{\prime \prime}, d^{\prime \prime}\right) \in \mathscr{A}$ and

$$
\operatorname{gcd}\left(m \ell_{1}, n \ell_{3}\right), \operatorname{gcd}\left(m \ell_{2}, n \ell_{4}\right) \in \mathscr{A} .
$$

For given $m, n$ we henceforth put

$$
L(m, n)=\left\{\boldsymbol{\ell} \in \mathbb{N}^{4}: \ell_{i} \mid(m n)^{\infty} \text { and (5.6), (5.7) hold }\right\} .
$$

It will be convenient to set

$$
m^{\prime \prime}=\operatorname{gcd}\left(a^{\prime \prime}, c^{\prime \prime}\right), \quad n^{\prime \prime}=\operatorname{gcd}\left(b^{\prime \prime}, d^{\prime \prime}\right),
$$

so that $m^{\prime \prime}, n^{\prime \prime} \in \mathscr{A}$ and $\operatorname{gcd}\left(m^{\prime \prime}, n^{\prime \prime}\right)=\operatorname{gcd}\left(m^{\prime \prime} n^{\prime \prime}, m n\right)=1$. We are now led to make the change of variables

$$
a^{\prime \prime}=m^{\prime \prime} a^{\prime \prime \prime}, \quad b^{\prime \prime}=n^{\prime \prime} b^{\prime \prime \prime}, \quad c^{\prime \prime}=m^{\prime \prime} c^{\prime \prime \prime}, \quad d^{\prime \prime}=n^{\prime \prime} d^{\prime \prime \prime},
$$

for $a^{\prime \prime \prime}, b^{\prime \prime \prime}, c^{\prime \prime \prime}, d^{\prime \prime \prime} \in \mathbb{N}$ satisfying

$$
\operatorname{gcd}\left(b^{\prime \prime \prime} d^{\prime \prime \prime}, m n m^{\prime \prime}\right)=\operatorname{gcd}\left(a^{\prime \prime \prime} c^{\prime \prime \prime}, m n n^{\prime \prime}\right)=1
$$

and

$$
\operatorname{gcd}\left(a^{\prime \prime \prime} d^{\prime \prime \prime}, b^{\prime \prime \prime} c^{\prime \prime \prime}\right)=1 \text {. }
$$


We will execute the asymptotic evaluation of $N(P), N_{\mathrm{loc}}(P)$ and $N_{\mathrm{Br}}(P)$ by estimating the number of allowable $a^{\prime \prime \prime}, b^{\prime \prime \prime}, c^{\prime \prime \prime}, d^{\prime \prime \prime}$ associated to given $\varepsilon_{2}, \varepsilon_{3}, \varepsilon_{4}, m, n, m^{\prime \prime}, n^{\prime \prime}$ and $\boldsymbol{\ell}$. In fact, for the treatments of $N_{\mathrm{loc}}(P)$ and $N_{\mathrm{Br}}(P)$ we will need to refine these transformations further, in order to extract the 2 -adic valuations of the variables.

Beginning with $N(P)$, our main goal in this section is a proof of the following result.

Lemma 5.2. - We have $N(P)=\tau P^{4}+O\left(P^{4-1 / 5+\varepsilon}\right)$, for any $\varepsilon>0$, where

$$
\tau=\frac{17}{16} \prod_{p \equiv 1(\bmod 4)} a_{p} \prod_{p \equiv 3(\bmod 4)}\left(\frac{1-\frac{1}{p}}{1+\frac{1}{p}}\right)^{2} b_{p},
$$

with $b_{p}$ given by (1.7) and

$$
a_{p}=\left(1-\frac{1}{p}\right)^{2}\left(1+\frac{2}{p}+\frac{1}{p^{2}}-\frac{2}{p^{4}}\right)
$$

Proof. - Our starting point is the modified version of (5.3), in which we have eliminated the constraint that $a d-b c \neq 0$ in the definition of $S_{\text {tot }}$. On multiplying through by $2^{3}$, we may work on the domain where $a, b, c, d$ are all positive. We now work through the changes of variables (5.4), (5.5) and (5.9), with $\varepsilon_{2}=\varepsilon_{3}=\varepsilon_{4}=+$. This leads to the expression

$$
N(P)=2 \sum_{\substack{m, n \in \mathscr{B} \\
\operatorname{gcd}(m, n)=1}} \sum_{\ell \in L(m, n)} \sum_{\begin{array}{r}
m^{\prime \prime}, n^{\prime \prime} \in \mathscr{A} \\
\operatorname{gcd}\left(m^{\prime \prime}, n^{\prime \prime}\right)=1 \\
\operatorname{gcd}\left(m^{\prime \prime} n^{\prime \prime}, m n\right)=1
\end{array}} N+O\left(P^{2+\varepsilon}\right),
$$

where $L(m, n)$ is given by (5.8) and $N$ denotes the number of $\left(a^{\prime \prime \prime}, b^{\prime \prime \prime}, c^{\prime \prime \prime}, d^{\prime \prime \prime}\right) \in \mathbb{N}^{4}$ such that (5.10) and (5.11) hold, with

$$
m m^{\prime \prime} \ell_{1} a^{\prime \prime \prime}, m n^{\prime \prime} \ell_{2} b^{\prime \prime \prime}, n m^{\prime \prime} \ell_{3} c^{\prime \prime \prime}, n n^{\prime \prime} \ell_{4} d^{\prime \prime \prime} \leqslant P .
$$

Let $K=K\left(m n, m^{\prime \prime}, n^{\prime \prime}\right)$ denote the set of $\mathbf{k} \in \mathbb{N}^{4}$ for which

$$
\operatorname{gcd}\left(k_{1} k_{4}, m n m^{\prime \prime} n^{\prime \prime}\right)=\operatorname{gcd}\left(k_{2}, m n n^{\prime \prime}\right)=\operatorname{gcd}\left(k_{3}, m n m^{\prime \prime}\right)=1 .
$$

We use the Möbius function to take care of (5.11), obtaining

$$
N=\sum_{\mathbf{k} \in K} \mu\left(k_{1}\right) \cdots \mu\left(k_{4}\right) N(\mathbf{k}),
$$

where $N(\mathbf{k})$ now denotes the number of $\left(a^{\prime \prime \prime}, b^{\prime \prime \prime}, c^{\prime \prime \prime}, d^{\prime \prime \prime}\right) \in \mathbb{N}^{4}$ such that (5.10) holds, with

$$
\begin{aligned}
m m^{\prime \prime} \ell_{1}\left[k_{1}, k_{2}\right] a^{\prime \prime \prime}, m n^{\prime \prime} \ell_{2}\left[k_{1}, k_{3}\right] b^{\prime \prime \prime} & \leqslant P, \\
n m^{\prime \prime} \ell_{3}\left[k_{2}, k_{4}\right] c^{\prime \prime \prime}, n n^{\prime \prime} \ell_{4}\left[k_{3}, k_{4}\right] d^{\prime \prime \prime} & \leqslant P .
\end{aligned}
$$

The estimation of $N(\mathbf{k})$ is straightforward, being based on counting integers in particular intervals which satisfy certain coprimality constraints. A trivial upper bound is given by

$$
N(\mathbf{k}) \ll \frac{P^{4}}{m^{2} n^{2} m^{\prime 2} n^{\prime 2} \ell_{1} \cdots \ell_{4}\left[k_{1}, k_{2}\right] \cdots\left[k_{3}, k_{4}\right]} .
$$

Note that

$$
\sum_{\ell \mid(m n)^{\infty}} \frac{1}{\ell^{\varepsilon}}=\frac{1}{\varphi_{\varepsilon}^{*}(m n)}
$$


for any $\varepsilon>0$, where $\varphi_{\varepsilon}^{*}$ is given by (2.10). The reciprocal of the latter is a function of $m n$ with average order $O(1)$. We may use these facts to restrict attention to parameters satisfying

$$
\max \left\{m, n, m^{\prime \prime}, n^{\prime \prime}, \ell_{i},\left[k_{i}, k_{j}\right]\right\} \leqslant T,
$$

for any $T \geqslant 1$, with overall error $O\left(T^{-1+\varepsilon} P^{4}\right)$.

Applying Lemma 5.1 with $q_{2}=1$ four times, we conclude that

$$
N(\mathbf{k})=\frac{\varphi^{*}\left(m n m^{\prime \prime}\right)^{2} \varphi^{*}\left(m n n^{\prime \prime}\right)^{2} P^{4}}{m^{2} n^{2} m^{\prime \prime 2} n^{\prime \prime 2} \ell_{1} \cdots \ell_{4}\left[k_{1}, k_{2}\right] \cdots\left[k_{3}, k_{4}\right]}\left(1+O\left(\frac{1}{T^{1-\varepsilon}}\right)\right),
$$

provided that $P \gg T^{5+3 \varepsilon}$. Substituting this estimate into (5.13) and (5.14), and extending the summation over the outer parameters to infinity, we are therefore led to the conclusion that

$$
N(P)=\tau P^{4}+O\left(P^{2+\varepsilon}+T^{-1+\varepsilon} P^{4}\right),
$$

provided that $P \gg T^{5+3 \varepsilon}$, where

$$
\begin{aligned}
\tau= & 2 \sum_{\substack{m, n \in \mathscr{B} \\
\operatorname{gcd}(m, n)=1}} \frac{1}{m^{2} n^{2}} \sum_{\ell \in L(m, n)} \frac{\varphi^{*}(m n)^{4}}{\ell_{1} \ell_{2} \ell_{3} \ell_{4}} \sum_{\begin{array}{c}
m^{\prime \prime}, n^{\prime \prime} \in \mathscr{A} \\
\operatorname{gcd}\left(m^{\prime \prime}, n^{\prime \prime}\right)=1 \\
\operatorname{gcd}\left(m^{\prime \prime} n^{\prime \prime}, m n\right)=1
\end{array}} \frac{\varphi^{*}\left(m^{\prime \prime}\right)^{2} \varphi^{*}\left(n^{\prime \prime}\right)^{2}}{m^{\prime \prime 2} n^{\prime \prime 2}} \\
& \times \sum_{\mathbf{k} \in K} \frac{\mu\left(k_{1}\right) \cdots \mu\left(k_{4}\right)}{\left[k_{1}, k_{2}\right] \cdots\left[k_{3}, k_{4}\right]} .
\end{aligned}
$$

Taking $T=P^{1 / 5-\varepsilon / 2}$ shows that the error term in this estimate is satisfactory for Lemma 5.2 and it remains to check that our value of $\tau$ agrees with what is recorded there.

To begin with one easily calculates

$$
\begin{aligned}
\sum_{\mathbf{k} \in K} \frac{\mu\left(k_{1}\right) \cdots \mu\left(k_{4}\right)}{\left[k_{1}, k_{2}\right] \cdots\left[k_{3}, k_{4}\right]} & =\prod_{p \nmid m n m^{\prime \prime} n^{\prime \prime}}\left(1-\frac{4}{p^{2}}+\frac{4}{p^{3}}-\frac{1}{p^{4}}\right) \prod_{p \mid m^{\prime \prime} n^{\prime \prime}}\left(1-\frac{1}{p^{2}}\right) \\
& =\prod_{p \nmid m n}\left(1-\frac{1}{p}\right)^{2}\left(1+\frac{2}{p}-\frac{1}{p^{2}}\right) \prod_{p \mid m^{\prime \prime} n^{\prime \prime}} \frac{\left(1+\frac{1}{p}\right)}{\left(1-\frac{1}{p}\right)\left(1+\frac{2}{p}-\frac{1}{p^{2}}\right)} .
\end{aligned}
$$

Introducing the sum over over $m^{\prime \prime}, n^{\prime \prime} \in \mathscr{A}$ such that $\operatorname{gcd}\left(m^{\prime \prime}, n^{\prime \prime}\right)=\operatorname{gcd}\left(m^{\prime \prime} n^{\prime \prime}, m n\right)=1$, we find that

$$
\sum_{m^{\prime \prime}, n^{\prime \prime}} \frac{\varphi^{*}\left(m^{\prime \prime}\right)^{2} \varphi^{*}\left(n^{\prime \prime}\right)^{2}}{m^{\prime \prime 2} n^{\prime \prime 2}} \prod_{p \mid m^{\prime \prime} n^{\prime \prime}} \frac{\left(1+\frac{1}{p}\right)}{\left(1-\frac{1}{p}\right)\left(1+\frac{2}{p}-\frac{1}{p^{2}}\right)}=\prod_{p \nmid m n}\left(\frac{1+\frac{2}{p}+\frac{1}{p^{2}}-\frac{2}{p^{4}}}{1+\frac{2}{p}-\frac{1}{p^{2}}}\right) .
$$

Next we note that

$$
\sum_{\ell \in L(m, n)} \frac{\varphi^{*}(m n)^{4}}{\ell_{1} \ell_{2} \ell_{3} \ell_{4}}=\prod_{p \mid m n}\left(1-\frac{1}{p}\right)^{2}\left(1+\frac{2}{p}+\frac{1}{p^{2}}-\frac{2}{p^{3}}\right)=\prod_{p \mid m n} c_{p},
$$


say, on recalling the definition (5.8) of $L(m, n)$. Let $a_{p}$ be given by (5.12) for any prime $p$. Putting this all together we conclude that

$$
\begin{aligned}
\tau & =2 \sum_{\substack{m, n \in \mathscr{B} \\
\operatorname{gcd}(m, n)=1}} \frac{1}{m^{2} n^{2}} \prod_{p \nmid m n} a_{p} \prod_{p \mid m n} c_{p} \\
& =2 a_{2} \prod_{p \equiv 1(\bmod 4)} a_{p} \prod_{p \equiv 3(\bmod 4)}\left(a_{p}+\frac{2 c_{p}}{p^{2}}\right) .
\end{aligned}
$$

Noting that $a_{2}=17 / 32$ and $a_{p}+\frac{2 c_{p}}{p^{2}}=\left(1-\frac{1}{p}\right)^{2}\left(1+\frac{1}{p}\right)^{-2} b_{p}$, with $b_{p}$ given by (1.7), this therefore concludes the proof of the lemma.

\section{Asymptotics: $N_{\text {loc }}(P)$}

Building on our work in the previous section we now turn to the resolution of Theorems 1.1 and 1.2. We begin with a few words about the local factors $\tau_{\text {loc, } v}$ that appear in the statement of the former. Each $\tau_{\text {loc }, v}$ may be interpreted as the density of points in $U\left(\mathbb{Q}_{v}\right) / G\left(\mathbb{Q}_{v}\right)$ for which the associated Châtelet surface has $\mathbb{Q}_{v}$-points. When $v=p$ is prime, this density is equal to

$$
\tau_{\text {loc }, p}=\lim _{k \rightarrow \infty} p^{-4 k} \#\left\{(a, b, c, d) \in A\left(p^{k}\right): \begin{array}{l}
X_{a, b, c, d}\left(\mathbb{Q}_{p}\right) \neq \emptyset \\
\min \left\{v_{p}(a), v_{p}(c)\right\} \leqslant 1 \\
\\
\min \left\{v_{p}(b), v_{p}(d)\right\} \leqslant 1 \\
\left.\min \left\{v_{p}(c), v_{p}(b)\right\} \leqslant \delta_{p}(d)\right\} \leqslant \delta_{p}
\end{array}\right\},
$$

where $A\left(p^{k}\right)=\left(\mathbb{Z} / p^{k} \mathbb{Z}\right)^{4} \backslash p\left(\mathbb{Z} / p^{k} \mathbb{Z}\right)^{4}$ and $\delta_{p}=1$ if $p \equiv 3(\bmod 4)$, with $\delta_{p}=0$ otherwise. Note that the condition $X_{a, b, c, d}\left(\mathbb{Q}_{p}\right) \neq \emptyset$ is precisely equivalent to the union of conditions issuing from parts (ii)-(iv) of Lemma 3.1. In particular, this condition is vacuous when $p \equiv 1(\bmod 4)$. Part (i) of Lemma 3.1 easily yields

$$
\tau_{\text {loc }, \infty}=\frac{7}{4},
$$

since there are 7 possible choices for the signs of $a, b, c, d$, with $a>0$ and $\sigma(b, c, d) \neq(+,-,-)$.

Our starting point is (5.1), followed by the changes of variables (5.4) and (5.5). It will be important to keep track of the 2-adic valuations of the variables involved. In particular we have already arranged things so that $a^{\prime \prime}$ is odd in $S_{\text {loc }}^{(\iota)}$. Let us write $\operatorname{gcd}\left(N_{1}, N_{2}\right)_{b}$ for the greatest odd common divisor of two integers $N_{1}$ and $N_{2}$. Instead of passing directly to (5.9), we set

$$
m^{\prime \prime}=\operatorname{gcd}\left(a^{\prime \prime}, c^{\prime \prime}\right), \quad n^{\prime \prime}=\operatorname{gcd}\left(b^{\prime \prime}, d^{\prime \prime}\right)_{b},
$$

so that $m^{\prime \prime}, n^{\prime \prime} \in \mathscr{A}$ and $\operatorname{gcd}\left(m^{\prime \prime}, n^{\prime \prime}\right)=\operatorname{gcd}\left(m^{\prime \prime} n^{\prime \prime}, 2 m n\right)=1$. Likewise, for $\iota \in\{0,1\}$ let us put

$$
L_{2}^{(\iota)}=\left\{(\beta, \gamma, \delta) \in \mathbb{Z}_{\geqslant 0}^{3}: \min \{\gamma, \delta\}=0, \min \{\beta, \delta\} \leqslant 1, \beta \geqslant 1-\iota\right\} .
$$

We are now led to make the change of variables

$$
a^{\prime \prime}=m^{\prime \prime} a^{\prime \prime \prime}, \quad b^{\prime \prime}=n^{\prime \prime} 2^{\beta} b^{\prime \prime \prime}, \quad c^{\prime \prime}=m^{\prime \prime} 2^{\gamma} c^{\prime \prime \prime}, \quad d^{\prime \prime}=n^{\prime \prime} 2^{\delta} d^{\prime \prime \prime},
$$

for $(\beta, \gamma, \delta) \in L_{2}^{(\iota)}$ and $a^{\prime \prime \prime}, b^{\prime \prime \prime}, c^{\prime \prime \prime}, d^{\prime \prime \prime} \in \mathbb{N}$ satisfying

$$
\operatorname{gcd}\left(b^{\prime \prime \prime} d^{\prime \prime \prime}, 2 m n m^{\prime \prime}\right)=\operatorname{gcd}\left(a^{\prime \prime \prime} c^{\prime \prime \prime}, 2 m n n^{\prime \prime}\right)=1
$$


and

$$
\operatorname{gcd}\left(a^{\prime \prime \prime} d^{\prime \prime \prime}, b^{\prime \prime \prime} c^{\prime \prime \prime}\right)_{b}=1 .
$$

The key difference between our analysis of $N(P)$ and $N_{\text {loc }}(P)$ is the use of Lemma 3.1. Part (i) of this result implies that we shall only be interested in $\varepsilon=\left(\varepsilon_{2}, \varepsilon_{3}, \varepsilon_{4}\right) \neq(+,-,-)$. Part (ii) ensures that $X_{a, b, c, d}\left(\mathbb{Q}_{p}\right) \neq \emptyset$ for any $p \nmid m n$. Now suppose that $p \mid m n$. Then according to part (iii) we will have $X_{a, b, c, d}\left(\mathbb{Q}_{p}\right) \neq \emptyset$ if and only if we do not have

$$
v_{p}\left(\ell_{1}\right)=v_{p}\left(\ell_{3}\right) \equiv 1(\bmod 2), \quad \text { or } \quad v_{p}\left(\ell_{2}\right)=v_{p}\left(\ell_{4}\right) \equiv 1(\bmod 2),
$$

but we do have $p \nmid a^{\prime \prime \prime} b^{\prime \prime \prime} c^{\prime \prime \prime} d^{\prime \prime \prime}$ and

$$
\left[\frac{-\varepsilon_{2} \ell_{1} \ell_{2} m^{\prime \prime} n^{\prime \prime} 2^{\beta} a^{\prime \prime \prime} b^{\prime \prime \prime}}{p}\right]+\left[\frac{-\varepsilon_{3} \varepsilon_{4} \ell_{3} \ell_{4} m^{\prime \prime} n^{\prime \prime} 2^{\gamma+\delta} c^{\prime \prime \prime} d^{\prime \prime \prime}}{p}\right] \geqslant 0,
$$

in the notation of (3.1). This last constraint is equivalent to demanding that $p \nmid a^{\prime \prime \prime} b^{\prime \prime \prime} c^{\prime \prime \prime} d^{\prime \prime \prime}$ and

$$
\left(\frac{-\varepsilon_{2} \ell_{1, p} \ell_{2, p} m^{\prime \prime} n^{\prime \prime} 2^{\beta} a^{\prime \prime \prime} b^{\prime \prime \prime}}{p}\right)+\left(\frac{-\varepsilon_{3} \varepsilon_{4} \ell_{3, p} \ell_{4, p} m^{\prime \prime} n^{\prime \prime} 2^{\gamma+\delta} c^{\prime \prime \prime} d^{\prime \prime \prime}}{p}\right) \geqslant 0,
$$

if $v_{p}\left(\ell_{i}\right) \in 2 \mathbb{Z}$ and $\ell_{i, p}=p^{-v_{p}\left(\ell_{i}\right)} \ell_{i}$, for $1 \leqslant i \leqslant 4$. In particular, for given $\beta, \gamma, \delta, \varepsilon, \ell, m^{\prime \prime}, n^{\prime \prime}$, these constraints force the vector $\left(a^{\prime \prime \prime}, b^{\prime \prime \prime}, c^{\prime \prime \prime}, d^{\prime \prime \prime}\right)$ to lie in one of finitely many congruence classes modulo $p$, for each $p \mid m n$. Let us denote the set of possible classes modulo $m n$ by

$$
T_{m n}(\ell) \cong \bigoplus_{p \mid m n} T_{p}(\ell)
$$

Here $T_{m n}(\ell) \subseteq(\mathbb{Z} / m n \mathbb{Z})^{4}$ and $T_{p}(\ell) \subseteq(\mathbb{Z} / p \mathbb{Z})^{4}$ for each prime $p$. The following result calculates their cardinality.

Lemma 6.1. - Let $p \mid m n$. Then we have

$$
\# T_{p}(\ell)= \begin{cases}\frac{3}{4}(p-1)^{4}, & \text { if } v_{p}\left(\ell_{i}\right) \in 2 \mathbb{Z} \text { for each } 1 \leqslant i \leqslant 4 \\ (p-1)^{4}, & \text { otherwise. }\end{cases}
$$

Proof. - Firstly, if $v_{p}\left(\ell_{i}\right) \notin 2 \mathbb{Z}$ for some $1 \leqslant i \leqslant 4$, then the only constraint modulo $p$ is that the coordinates should all be coprime to $p$. The claim is therefore obvious in this case. If $v_{p}\left(\ell_{i}\right) \in 2 \mathbb{Z}$ for each $1 \leqslant i \leqslant 4$, then for given $\varepsilon_{2}, \ell_{1, p}, \ell_{2, p}, m^{\prime \prime}, n^{\prime \prime}$ not divisible by $p$, it is clear that there are $\frac{1}{2}(p-1)^{2}$ choices for $a^{\prime \prime \prime}, b^{\prime \prime \prime}$ modulo $p$ such that

$$
\left(\frac{-\varepsilon_{2} \ell_{1, p} \ell_{2, p} m^{\prime \prime} n^{\prime \prime} 2^{\beta} a^{\prime \prime \prime} b^{\prime \prime \prime}}{p}\right)=1 .
$$

Likewise there are $\frac{1}{2}(p-1)^{2}$ choices of $c^{\prime \prime \prime}, d^{\prime \prime \prime}$ modulo $p$ for which

$$
\left(\frac{-\varepsilon_{3} \varepsilon_{4} \ell_{3, p} \ell_{4, p} m^{\prime \prime} n^{\prime \prime} 2^{\gamma+\delta} c^{\prime \prime \prime} d^{\prime \prime \prime}}{p}\right)=1,
$$

whence we arrive at the desired expression on subtracting the contribution from $a^{\prime \prime \prime}, b^{\prime \prime \prime}, c^{\prime \prime \prime}, d^{\prime \prime \prime}$ modulo $p$ for which both Legendre symbols are 1 . 
We need to restrict attention to $\ell \in L(m, n)$ for which (6.7) does not hold for any $p \mid m n$. We will capture the latter constraint by writing $\neg(6.7)$, where $\neg$ is the symbol used for logical negation. Summarising our investigation so far, our analogue of (5.13) is

$$
\begin{aligned}
& N_{\text {loc }}(P)=\frac{1}{4} \sum_{\iota \in\{0,1\}} \sum_{\varepsilon \in\{ \pm\}^{3} \backslash(+,-,-)} \sum_{\substack{m, n \in \mathscr{B} \\
\operatorname{gcd}(m, n)=1}} \sum_{\substack{\ell \in L(m, n) \\
\neg(6.7)}} \\
& \times \sum_{\substack{m^{\prime \prime}, n^{\prime \prime} \in \mathscr{A} \\
\operatorname{gcd}\left(m^{\prime \prime}, n^{\prime \prime}\right)=1 \\
\operatorname{gcd}\left(m^{\prime \prime} n^{\prime \prime}, 2 m n\right)=1}} \sum_{\substack{\beta, \gamma, \delta) \in L_{2}^{(\iota)}\\
}} N_{\mathrm{loc}}+O\left(P^{2+\varepsilon}\right),
\end{aligned}
$$

where $L(m, n)$ (resp. $\left.L_{2}^{(\iota)}\right)$ is given by (5.8) (resp. (6.3) ) and $N_{\text {loc }}$ denotes the total number of $\left(a^{\prime \prime \prime}, b^{\prime \prime \prime}, c^{\prime \prime \prime}, d^{\prime \prime \prime}\right) \in \mathbb{N}^{4}$ such that (6.5) and (6.6) hold, with

$$
m m^{\prime \prime} \ell_{1} a^{\prime \prime \prime}, m n^{\prime \prime} \ell_{2} 2^{\beta} b^{\prime \prime \prime}, n m^{\prime \prime} \ell_{3} 2^{\gamma} c^{\prime \prime \prime}, n n^{\prime \prime} \ell_{4} 2^{\delta} d^{\prime \prime \prime} \leqslant P,
$$

$X_{a, b, c, d}\left(\mathbb{Q}_{2}\right) \neq \emptyset$ and $\left(a^{\prime \prime \prime}, b^{\prime \prime \prime}, c^{\prime \prime \prime}, d^{\prime \prime \prime}\right) \in T_{m n}(\ell)(\bmod m n)$.

As before we use the Möbius function to detect the condition (6.6). Recall the definition of $K=K\left(m n, m^{\prime \prime}, n^{\prime \prime}\right)$ from $₫ 5$ and define $K^{\prime}=K^{\prime}\left(m n, m^{\prime \prime}, n^{\prime \prime}\right)$ to be the subset of $\mathbf{k} \in K$ for which $2 \nmid k_{1} k_{2} k_{3} k_{4}$. It will be convenient to set

$$
\begin{array}{ll}
A=m m^{\prime \prime} \ell_{1}\left[k_{1}, k_{2}\right], & B=m n^{\prime \prime} \ell_{2} 2^{\beta}\left[k_{1}, k_{3}\right], \\
C=n m^{\prime \prime} \ell_{3} 2^{\gamma}\left[k_{2}, k_{4}\right], & D=n n^{\prime \prime} \ell_{4} 2^{\delta}\left[k_{3}, k_{4}\right] .
\end{array}
$$

Then

$$
N_{\mathrm{loc}}=\sum_{\mathbf{k} \in K^{\prime}} \mu\left(k_{1}\right) \cdots \mu\left(k_{4}\right) N_{\mathrm{loc}}(\mathbf{k}),
$$

where $N_{\text {loc }}(\mathbf{k})$ is now the number of $\left(a^{\prime \prime \prime}, b^{\prime \prime \prime}, c^{\prime \prime \prime}, d^{\prime \prime \prime}\right) \in \mathbb{N}^{4}$ such that (6.5) holds, with

$$
A a^{\prime \prime \prime}, B b^{\prime \prime \prime}, C c^{\prime \prime \prime}, D d^{\prime \prime \prime} \leqslant P,
$$

$X_{a, b, c, d}\left(\mathbb{Q}_{2}\right) \neq \emptyset$ and

$$
\left(\left[k_{1}, k_{2}\right] a^{\prime \prime \prime}, \ldots,\left[k_{3}, k_{4}\right] d^{\prime \prime \prime}\right) \in T_{m n}(\ell)(\bmod m n) .
$$

Before estimating $N_{\text {loc }}(\mathbf{k})$, it remains to interpret the constraint $X_{a, b, c, d}\left(\mathbb{Q}_{2}\right) \neq \emptyset$, as described in part (iv) of Lemma 3.1. An inspection of Lemmas 4.8 4.15 shows that for a given choice of $(\beta, \gamma, \delta) \in L_{2}^{(\iota)}$, the 2-adic constraints placed on $\left(a^{\prime \prime \prime}, b^{\prime \prime \prime}, c^{\prime \prime \prime}, d^{\prime \prime \prime}\right)$ constitute a union of particular congruence classes modulo 16. Let us denote by $H_{\beta, \gamma, \delta}$ the set of all possible classes modulo 16 that can arise. Then the constraint $X_{a, b, c, d}\left(\mathbb{Q}_{2}\right) \neq \emptyset$ in $N(\mathbf{k})$ is equivalent to demanding that

$$
\left(a^{\prime \prime \prime}, b^{\prime \prime \prime}, c^{\prime \prime \prime}, d^{\prime \prime \prime}\right) \in H_{\beta, \gamma, \delta}(\bmod 16) .
$$

Recall the definition (6.3) of $L_{2}^{(\iota)}$. Note that $H_{\beta, \gamma, \delta}$ depends on $\varepsilon, \mathbf{k}, \boldsymbol{\ell}, m, n, m^{\prime \prime}, n^{\prime \prime}$ in addition to $\beta, \gamma, \delta$, but the cardinality of this set does not depend on these additional parameters, being equal to the total number of $\left(A^{\prime}, B^{\prime}, C^{\prime}, D^{\prime}\right) \in(\mathbb{Z} / 16 \mathbb{Z})^{4}$, with $2 \nmid A^{\prime} B^{\prime} C^{\prime} D^{\prime}$, for which $\left(A^{\prime}, 2^{\beta} B^{\prime}, 2^{\gamma} C^{\prime}, 2^{\delta} D^{\prime}\right)$ belongs to $T_{\text {tot }}^{(2)}$ modulo 16 , in the notation of $\$ 4.4$. A little thought therefore reveals that

$$
\sum_{\iota \in\{0,1\}} \sum_{(\beta, \gamma, \delta) \in L_{2}^{(\iota)}} \frac{\# H_{\beta, \gamma, \delta}}{2^{16+\beta+\gamma+\delta}}=\tau_{\mathrm{loc}, 2}
$$


where $\tau_{\text {loc }, 2}$ is given by (6.1). We will give a numerical value for $\tau_{\text {loc, }, 2}$ in Lemma 6.2.

The estimation of $N_{\text {loc }}(\mathbf{k})$ is straightforward, being based on Lemma 5.1. A trivial upper bound is given by $N_{\text {loc }}(\mathbf{k}) \ll P^{4} /(A B C D)$. As previously, this shows that we may restrict attention to parameters satisying

$$
\max \left\{m, n, m^{\prime \prime}, n^{\prime \prime}, \ell_{i},\left[k_{i}, k_{j}\right], 2^{\beta}, 2^{\gamma}, 2^{\delta}\right\} \leqslant T,
$$

with error $O\left(T^{-1+\varepsilon} P^{4}\right)$. Having reduced the size of the parameters suitably we turn to an asymptotic formula for $N_{\text {loc }}(\mathbf{k})$. The coprimality conditions $\operatorname{gcd}\left(a^{\prime \prime \prime} b^{\prime \prime \prime} c^{\prime \prime \prime} d^{\prime \prime \prime}, 2 m n\right)=1$ in (6.5) will automatically be taken care of by (6.10) and (6.11). This time we will employ Lemma 5.1 with $q_{1} \in\left\{m^{\prime \prime}, n^{\prime \prime}\right\}, q_{2}=16 m n$ and $x \in\{P / A, \ldots, P / D\}$. Under the assumption $P \gg T^{8+\varepsilon}$ we therefore obtain

$$
N_{\mathrm{loc}}(\mathbf{k})=\frac{\varphi^{*}\left(m^{\prime \prime}\right)^{2} \varphi^{*}\left(n^{\prime \prime}\right)^{2} P^{4}}{A B C D} \cdot \frac{\# T_{m n}(\ell)}{(m n)^{4}} \cdot \frac{\# H_{\beta, \gamma, \delta}}{2^{16}}\left(1+O\left(\frac{1}{T^{1-\varepsilon}}\right)\right) .
$$

We take $T=P^{1 / 8-\varepsilon / 2}$ and substitute this estimate into (6.8) and (6.9), extending the summation over the outer parameters to infinity. This concludes the statement of Theorem 1.1, with

$$
\begin{aligned}
& \tau_{\mathrm{loc}}=\frac{\tau_{\mathrm{loc}, 2}}{4} \sum_{\varepsilon \in\{ \pm\}^{3} \backslash(+,-,-)} \sum_{\substack{m, n \in \mathscr{B} \\
\operatorname{gcd}(m, n)=1}} \frac{1}{m^{2} n^{2}} \sum_{\substack{\ell \in L(m, n) \\
\neg \sqrt{6.7)}}} \frac{\# T_{m n}(\ell)}{\ell_{1} \ell_{2} \ell_{3} \ell_{4}(m n)^{4}} \\
& \times \sum_{\substack{m^{\prime \prime}, n^{\prime \prime} \in \mathscr{A} \\
\operatorname{gcd}\left(m^{\prime \prime}, n^{\prime \prime}\right)=1 \\
\operatorname{gcd}\left(m^{\prime \prime} n^{\prime \prime}, 2 m n\right)=1}} \frac{\varphi^{*}\left(m^{\prime \prime}\right)^{2} \varphi^{*}\left(n^{\prime \prime}\right)^{2}}{m^{\prime \prime 2} n^{\prime \prime 2}} \sum_{\mathbf{k} \in K^{\prime}} \frac{\mu\left(k_{1}\right) \cdots \mu\left(k_{4}\right)}{\left[k_{1}, k_{2}\right] \cdots\left[k_{3}, k_{4}\right]} .
\end{aligned}
$$

It remains to check that this constant satisfies the description given in the statement of the theorem.

The calculations at the close of $\$ 5$ immediately yield

$$
\sum_{m^{\prime \prime}, n^{\prime \prime}} \frac{\varphi^{*}\left(m^{\prime \prime}\right)^{2} \varphi^{*}\left(n^{\prime \prime}\right)^{2}}{m^{\prime \prime 2} n^{\prime \prime 2}} \sum_{\mathbf{k} \in K^{\prime}} \frac{\mu\left(k_{1}\right) \cdots \mu\left(k_{4}\right)}{\left[k_{1}, k_{2}\right] \cdots\left[k_{3}, k_{4}\right]}=\prod_{p \nmid 2 m n} a_{p},
$$

with $a_{p}$ given by (5.12). For given $i, j \in\{0,1\}$, with $\min \{i, j\}=0$, let

$$
E(i, j)=\left\{\begin{array}{ll} 
& \min \left\{\nu_{1}, \nu_{2}\right\}=\min \left\{\nu_{3}, \nu_{4}\right\}=0 \\
\boldsymbol{\nu} \in \mathbb{Z}_{\geqslant 0}^{4}: & \min \left\{i+\nu_{1}, j+\nu_{3}\right\} \leqslant 1 \\
& \nu_{1}=\nu_{3} \Rightarrow \nu_{1}=\nu_{3}=0 \\
\nu_{2}=\nu_{4} \Rightarrow \nu_{2}=\nu_{4}=0
\end{array}\right\} .
$$

The conditions in this set arise from the constraints (5.6), (5.7) in the definition of $L(m, n)$, together with the constraint that (6.7) should not hold. Applying Lemma 6.1, we deduce that

$$
\sum_{\substack{\ell \in L(m, n) \\ \neg(6.7)}} \frac{\# T_{m n}(\ell)}{\ell_{1} \ell_{2} \ell_{3} \ell_{4}(m n)^{4}}=\prod_{p \mid m n} c_{p}^{\prime}
$$


where

$$
\begin{aligned}
c_{p}^{\prime} & =\sum_{\boldsymbol{\nu} \in E\left(v_{p}(m), v_{p}(n)\right)} \frac{\# T_{p}\left(p^{\nu_{1}}, p^{\nu_{2}}, p^{\nu_{3}}, p^{\nu_{4}}\right)}{p^{4+\nu_{1}+\nu_{2}+\nu_{3}+\nu_{4}}} \\
& =\left(1-\frac{1}{p}\right)^{4} \sum_{\boldsymbol{\nu} \in E\left(v_{p}(m), v_{p}(n)\right)} \frac{(3 / 4)^{f(\boldsymbol{\nu})}}{p^{\nu_{1}+\nu_{2}+\nu_{3}+\nu_{4}}},
\end{aligned}
$$

for any $p \mid m n$, where

$$
f(\boldsymbol{\nu})= \begin{cases}1, & \text { if } \nu_{i} \in 2 \mathbb{Z} \text { for } 1 \leqslant i \leqslant 4, \\ 0, & \text { otherwise. }\end{cases}
$$

A straightforward calculation reveals that

$$
\begin{aligned}
c_{p}^{\prime} & =\left(1-\frac{1}{p}\right)^{4}\left(1+\frac{4+\frac{3}{p}+\frac{2}{p^{2}}+\frac{2}{p^{3}}}{p\left(1-\frac{1}{p^{2}}\right)}+\frac{2+\frac{4}{p}+\frac{3}{2 p^{2}}}{p^{2}\left(1-\frac{1}{p^{2}}\right)^{2}}\right) \\
& =\left(1-\frac{1}{p}\right)^{2}\left(1+\frac{1}{p}\right)^{-2}\left(1+\frac{4}{p}+\frac{3}{p^{2}}+\frac{2}{p^{3}}+\frac{3 / 2}{p^{4}}-\frac{2}{p^{5}}-\frac{2}{p^{6}}\right) .
\end{aligned}
$$

Returning to our expression for $\tau_{\text {loc }}$ it remains to execute the summation over $\varepsilon$ and $m, n$. The latter leads to the identity

$$
\sum_{\substack{m, n \in \mathscr{B} \\ \operatorname{gcd}(m, n)=1}} \frac{1}{m^{2} n^{2}} \prod_{p \nmid 2 m n} a_{p} \prod_{p \mid m n} c_{p}^{\prime}=\prod_{p \equiv 1(\bmod 4)} a_{p} \prod_{p \equiv 3(\bmod 4)}\left(a_{p}+\frac{2 c_{p}^{\prime}}{p^{2}}\right) .
$$

Carrying out the sum over $\varepsilon$, and recalling (6.2), we are therefore led to the final expression

$$
\tau_{\mathrm{loc}}=\tau_{\mathrm{loc}, \infty} \tau_{\mathrm{loc}, 2} \prod_{p \equiv 1(\bmod 4)} a_{p} \prod_{p \equiv 3(\bmod 4)}\left(a_{p}+\frac{2 c_{p}^{\prime}}{p^{2}}\right),
$$

with $a_{p}, c_{p}^{\prime}$ given by (5.12) and (6.13), respectively.

Now it is easy to check that $a_{p}=\tau_{\text {loc }, p}$ in (6.1) when $p \equiv 1(\bmod 4)$. In order to complete the proof of Theorem 1.1 it therefore remains to show that

$$
a_{p}+\frac{2 c_{p}^{\prime}}{p^{2}}=\tau_{\text {loc }, p},
$$

when $p \equiv 3(\bmod 4)$. To see this we may split the calculation of $\tau_{\text {loc, } p}$ into three densities, according to whether $p \nmid m n$ or $p \mid m$ or $p \mid n$. The density corresponding to the first case is $a_{p}$. We claim that the density $d_{p}$, say, corresponding to the second case is $c_{p}^{\prime} / p^{2}$. This will suffice to establish the desired identity, since the third density follows by symmetry. Recalling (6.1) it is clear that

$$
\begin{aligned}
d_{p}=\frac{1}{p^{2}} \lim _{k \rightarrow \infty} p^{-4 k} \#\left\{\left(a^{\prime}, b^{\prime}, c, d\right) \in\left(\mathbb{Z} / p^{k} \mathbb{Z}\right)^{4}: \begin{array}{l}
X_{p a^{\prime}, p b^{\prime}, c, d}\left(\mathbb{Q}_{p}\right) \neq \emptyset \\
\min \left\{1+v_{p}\left(a^{\prime}\right), v_{p}(c)\right\} \leqslant 1 \\
\min \left\{1+v_{p}\left(b^{\prime}\right), v_{p}(d)\right\} \leqslant 1 \\
\left.\min \left\{v_{p}(c), v_{p}(b)\right\} \leqslant \delta_{p}(d)\right\}=0
\end{array}\right\} \\
=\frac{1}{p^{2}} \sum_{\boldsymbol{\nu} \in E(1,0)} \frac{\# T_{p}\left(p^{\nu_{1}}, p^{\nu_{2}}, p^{\nu_{3}}, p^{\nu_{4}}\right)}{p^{4+\nu_{1}+\nu_{2}+\nu_{3}+\nu_{4}}},
\end{aligned}
$$


in the notation of (6.12). It is now clear that $d_{p}=c_{p}^{\prime} / p^{2}$, as claimed.

The remainder of this section is concerned with the proof of Theorem 1.2. There are two ingredients to this. The first is a numerical evaluation of the constant $\tau_{\text {loc }}$ in (6.14) and the second is the asymptotic estimate for $N(P)$ in Lemma 5.2. Beginning with the former, we have the following calculation of the 2 -adic density $\tau_{\text {loc }, 2}$ in (6.1).

Lemma 6.2. - We have $\tau_{\mathrm{loc}, 2}=4751 /\left(9 \times 2^{10}\right)$.

Proof. - Let $(i, j) \in(\mathbb{Z} / 4 \mathbb{Z})^{2}$, with $2 \nmid i$. To establish the lemma it will be convenient to set

$$
\tau_{2}(i, j)=\sum_{(\beta, \gamma, \delta) \in L_{2}^{(1)}} \frac{\# H_{\beta, \gamma, \delta}(i, j)}{2^{16+\beta+\gamma+\delta}},
$$

where $L_{2}^{(1)}$ is given by (6.3) and $\# H_{\beta, \gamma, \delta}(i, j)$ is the number of $\left(A^{\prime}, B^{\prime}, C^{\prime}, D^{\prime}\right) \in(\mathbb{Z} / 16 \mathbb{Z})^{4}$, with $2 \nmid A^{\prime} B^{\prime} C^{\prime} D^{\prime}$, for which $\left(A^{\prime}, 2^{\beta} B^{\prime}, 2^{\gamma} C^{\prime}, 2^{\delta} D^{\prime}\right)$ belongs to $T_{\text {tot }}^{(2)}$ modulo 16 and

$$
\left(A^{\prime}, 2^{\beta} B^{\prime}\right) \equiv(i, j)(\bmod 4) .
$$

We note that $\tau_{2}(i, j)=\tau_{2}(-i,-j)$. Hence we have

$$
\begin{aligned}
\tau_{\text {loc }, 2} & =\sum_{i \in\{1,3\}} \sum_{j \in\{0,2\}} \tau_{2}(i, j)+\sum_{i \in\{1,3\}} \sum_{j \in\{0,1,2,3\}} \tau_{2}(i, j) \\
& =2 \sum_{i \in\{1,3\}} \sum_{j \in\{0,2\}} \tau_{2}(i, j)+\sum_{i, j \in\{1,3\}} \tau_{2}(i, j) \\
& =4\left(\tau_{2}(1,0)+\tau_{2}(1,2)\right)+2\left(\tau_{2}(1,1)+\tau_{2}(1,3)\right) .
\end{aligned}
$$

The calculation of the densities $\tau_{2}(i, j)$ is based on our analysis in 44.4 The process is routine but very tedious. We have decided merely to record the outcome of the investigation in Table 6. Once combined with our formula for $\tau_{\mathrm{loc}, 2}$, this therefore concludes the proof of the

\begin{tabular}{|l|l|l|}
\hline$(i, j)(\bmod 4)$ & $\tau_{2}(i, j)$ & Proof \\
\hline$(1,0)$ & $89 /\left(9 \times 2^{8}\right)$ & Lemma 4.12 \\
$(1,1)$ & $17 /\left(3 \times 2^{7}\right)$ & Lemma 4.8 \\
$(1,2)$ & $23 / 2^{9}$ & Lemma 4.14 \\
$(1,3)$ & $95 / 2^{11}$ & Lemma 4.11 \\
\hline
\end{tabular}

TABLE 6. 2-adic densities $\tau_{2}(i, j)$

lemma.

The numerical value of $\tau_{\text {loc,2 }}$ is $0.515516 \cdots$. Let $N\left(2^{k}\right)$ denote the cardinality on the right hand side of (6.1) when $p=2$. Tim Dokchitser has kindly implemented a computer algorithm for calculating the ratios $2^{-4 k} N\left(2^{k}\right)$ for small values of $k$. Taking $k \leqslant 7$ leads to the numerical value $0.514905 \cdots$, which agrees quite closely with Lemma 6.2.

The conclusion of Theorem 1.2 is now available. Combining Theorem 1.1 and Lemma 5.2 , we conclude that

$$
\lim _{P \rightarrow \infty} \frac{N_{\mathrm{loc}}(P)}{N(P)}=\frac{\tau_{\mathrm{loc}}}{\tau}
$$


with $\tau_{\text {loc }}$ as in (6.14). It follows from (5.12) and (6.13) that

$$
a_{p}+\frac{2 c_{p}^{\prime}}{p^{2}}=\frac{\left(1-\frac{1}{p}\right)^{2}}{\left(1+\frac{1}{p}\right)^{2}}\left(1+\frac{4}{p}+\frac{8}{p^{2}}+\frac{12}{p^{3}}+\frac{5}{p^{4}}+\frac{1}{p^{6}}-\frac{4}{p^{7}}-\frac{4}{p^{8}}\right) .
$$

Applying (6.2) and Lemma 6.2, we deduce that

$$
\begin{aligned}
\frac{\tau_{\text {loc }}}{\tau} & =\frac{\tau_{\text {loc }, \infty} \tau_{\text {loc }, 2}}{17 / 16} \prod_{p \equiv 3(\bmod 4)} \frac{\left(1+\frac{1}{p}\right)^{2}\left(a_{p}+\frac{2 c_{p}^{\prime}}{p^{2}}\right)}{\left(1-\frac{1}{p}\right)^{2} b_{p}} \\
& =\frac{7 \times 4751}{2^{8} \times 3^{2} \times 17} \prod_{p \equiv 3(\bmod 4)}\left(\frac{1+\frac{4}{p}+\frac{8}{p^{2}}+\frac{12}{p^{3}}+\frac{5}{p^{4}}+\frac{1}{p^{6}}-\frac{4}{p^{7}}-\frac{4}{p^{8}}}{b_{p}}\right) \\
& =\frac{33257}{39168} \prod_{p \equiv 3(\bmod 4)}\left(1-\frac{6-\frac{9}{p^{2}}+\frac{4}{p^{4}}}{p^{4} b_{p}}\right),
\end{aligned}
$$

with $b_{p}$ given by (1.7). The latter Euler product converges very rapidly and has numerical value $0.98186722 \cdots$, whence $\tau_{\text {loc }} / \tau=0.8336897$, up to 7 decimal places. This completes the proof of Theorem 1.2 .

\section{Asymptotics: $N_{\mathrm{Br}}(P)$}

The goal of this section is to establish Theorem 1.3, The argument will begin along similar lines to the treatments of $N(P)$ and $N_{\text {loc }}(P)$, but the analysis is ultimately more involved. Whereas our earlier work relied upon the basic estimate in Lemma 5.1 for the number of integers in an interval which are coprime to a given integer, the treatment of $N_{\mathrm{Br}}(P)$ will require more sophisticated tools from analytic number theory.

7.1. Preliminary analytic tools. - Let $\mathbf{a}=\left(a_{1}, a_{2}\right), \mathbf{q}=\left(q_{1}, q_{2}\right) \in \mathbb{N}^{2}$. We define an arithmetic function $g_{\mathbf{a}, \mathbf{q}}: \mathbb{N} \rightarrow \mathbb{R}$ multiplicatively on prime powers via

$$
g_{\mathbf{a}, \mathbf{q}}\left(p^{\nu}\right)= \begin{cases}1, & \text { if } \nu=0, \\ \frac{1}{2}\left(\frac{a_{1} a_{2} q_{1}}{p}\right)-\frac{1}{2}, & \text { if } \nu=1, p \equiv 3(\bmod 4) \text { and } p \nmid a_{1} a_{2} q_{1} q_{2}, \\ 0, & \text { otherwise. }\end{cases}
$$

Let

$$
\chi_{q_{2}}(z)= \begin{cases}1, & \text { if } z \in \mathscr{B} \text { with } \operatorname{gcd}\left(z, q_{2}\right)=1 \\ 0, & \text { otherwise }\end{cases}
$$

where $\mathscr{B}$ is given by (2.1). One finds that

$$
g_{\mathbf{a}, \mathbf{q}}(z)=\frac{\mu(z) \chi_{q_{2}}(z)}{2^{\omega(z)}} \sum_{\ell \mid z} \mu(\ell)\left(\frac{a_{1} a_{2} q_{1}}{\ell}\right) .
$$

We begin with an estimate for the average order of $\left|g_{\mathbf{a}, \mathbf{q}}\right|$. 
Lemma 7.1. - Let $\varepsilon>0$. For $x_{1}, x_{2}, Z>2$, we have

$$
\sum_{a_{1} \leqslant x_{1}} \sum_{a_{2} \leqslant x_{2}} \sum_{\substack{z \leqslant Z \\ \operatorname{gcd}\left(z, q_{1}\right)=1}}\left|g_{\mathbf{a}, \mathbf{q}}(z)\right| \ll \frac{x_{1} x_{2} Z}{(\log Z)^{3 / 4}}+\sqrt{x_{1} x_{2}} Z^{11 / 8+\varepsilon},
$$

uniformly in $q_{1}, q_{2}$.

Proof. - Applying the Burgess bound for character sums, it follows that

$$
\sum_{a_{1} \leqslant x_{1}} \sum_{a_{2} \leqslant x_{2}}\left(\frac{a_{1} a_{2}}{\ell}\right) \ll \sqrt{x_{1} x_{2}} \ell^{3 / 8+\varepsilon}
$$

for any integer $\ell>1$. If $\chi_{q_{2}}(z)=1$ then (7.2) implies that

$$
\left|g_{\mathbf{a}, \mathbf{q}}(z)\right|=\frac{1}{2^{\omega(z)}} \sum_{\ell \mid z} \mu(\ell)\left(\frac{a_{1} a_{2} q_{1}}{\ell}\right) .
$$

Hence

$$
\begin{aligned}
\sum_{a_{1} \leqslant x_{1}} \sum_{a_{2} \leqslant x_{2}} \sum_{\substack{z \leqslant Z \\
\operatorname{gcd}\left(z, q_{1}\right)=1}}\left|g_{\mathbf{a}, \mathbf{q}}(z)\right| & =\sum_{a_{1} \leqslant x_{1}} \sum_{a_{2} \leqslant x_{2}} \sum_{\substack{z \leqslant Z \\
\operatorname{gcd}\left(z, q_{1} q_{2}\right)=1 \\
z \in \mathscr{B}}} \frac{1}{2^{\omega(z)}}+O\left(\sqrt{x_{1} x_{2}} Z^{11 / 8+\varepsilon}\right) \\
& \ll x_{1} x_{2} \sum_{\substack{z \leqslant Z \\
z \in \mathscr{B}}} \frac{1}{2^{\omega(z)}}+\sqrt{x_{1} x_{2}} Z^{11 / 8+\varepsilon} .
\end{aligned}
$$

The remaining sum over $z$ is easily seen to be $O\left(Z /(\log Z)^{3 / 4}\right)$, by an application of the Selberg-Delange method (see [10, §II.5], for example). This concludes the proof.

At a certain point in our argument it will be useful to approximate the function $g_{\mathbf{a}, \mathbf{q}}$ in (7.2) by the simpler arithmetic function

$$
\hat{g}_{q_{2}}(z)=\frac{\mu(z) \chi_{q_{2}}(z)}{2^{\omega(z)}} .
$$

We will need an asymptotic formula for the average order of $\hat{g}_{q_{2}}(z) h(z) / z$, where $h$ is an arbitrary multiplicative arithmetic function satisfying

$$
h(p)=1+O\left(\frac{1}{p}\right)
$$

for primes $p$. This is achieved in the following result.

Lemma 7.2. - Let $\varepsilon>0$ and let $h$ be a multiplicative arithmetic function satisfying (7.4). For $Z \geqslant 2$ and $q_{2} \in \mathbb{N}$, we have

$$
\sum_{\substack{z \leqslant Z \\ \operatorname{gcd}\left(z, q_{2}\right)=1}} \frac{\hat{g}_{q_{2}}(z) h(z)}{z}=\frac{4 c(h) \gamma\left(q_{2} ; h\right)}{|\Gamma(-1 / 4)|(\log Z)^{1 / 4}}\left\{1+O\left(\frac{q_{2}^{\varepsilon}}{\log Z}\right)\right\},
$$

where

$$
\gamma\left(q_{2} ; h\right)=\prod_{\substack{p \equiv 3(\bmod 4) \\ p \mid q_{2}}}\left(1-\frac{h(p)}{2 p}\right)^{-1}
$$


and $c(h)$ is the convergent Euler product

$$
c(h)=\prod_{p}\left(1-\frac{1}{p}\right)^{-1 / 4} \prod_{p \equiv 3(\bmod 4)}\left(1-\frac{h(p)}{2 p}\right) .
$$

Proof. - We consider the associated Dirichlet series

$$
\begin{aligned}
& D(s)=\sum_{\substack{z=1 \\
\operatorname{gcd}\left(z, q_{2}\right)=1}}^{\infty} \frac{\hat{g}_{q_{2}}(z) h(z)}{z^{s}}=\prod_{\substack{p \equiv 3(\bmod 4) \\
p \nmid q_{2}}}\left(1-\frac{h(p)}{2 p^{s}}\right) \\
& =\prod_{p \equiv 3(\bmod 4)}\left(1-\frac{h(p)}{2 p^{s}}\right) \prod_{\substack{p \equiv 3(\bmod 4) \\
p \mid q_{2}}}\left(1-\frac{h(p)}{2 p^{s}}\right)^{-1} \\
& =D_{1}(s) \prod_{\substack{p \equiv 3(\bmod 4) \\
p \mid q_{2}}}\left(1-\frac{h(p)}{2 p^{s}}\right)^{-1},
\end{aligned}
$$

say, for $\Re(s)=\sigma>1$. Since $h(p)=1+O(1 / p)$, one sees that

$$
\begin{aligned}
& \zeta(s) D_{1}(s)^{4}=\prod_{p \neq \equiv 3(\bmod 4)}\left(1-\frac{1}{p^{s}}\right)^{-1} \prod_{p \equiv 3(\bmod 4)}\left(1-\frac{1}{p^{s}}\right)^{-1}\left(1-\frac{2}{p^{s}}+O\left(\frac{1}{p^{2 \sigma}}+\frac{1}{p^{\sigma+1}}\right)\right) \\
& =L(s, \chi) G(s) \text {, }
\end{aligned}
$$

where $L(s, \chi)$ is the Dirichlet $L$-function associated to the real character $\chi$ modulo 4 and $G$ may be continued as a holomorphic function to the half-plane $\sigma>1 / 2$ and is bounded absolutely in this region. Hence it follows that $D(s)=\zeta(s)^{-1 / 4} G_{q_{2}}(s)$, where

$$
G_{q_{2}}(s)=L(s, \chi)^{1 / 4} G(s)^{1 / 4} \prod_{\substack{p \equiv 3(\bmod 4) \\ p \mid q_{2}}}\left(1-\frac{h(p)}{2 p^{s}}\right)^{-1} .
$$

In particular $G_{q_{2}}(1)=c(h) \gamma\left(q_{2} ; h\right)$, in the notation of the lemma.

We now invoke the Selberg-Delange method, as described in Tenenbaum [10, §II.5]. This implies that

$$
\sum_{\substack{z \leqslant Z \\ \operatorname{gcd}\left(z, q_{2}\right)=1}} \hat{g}_{q_{2}}(z) h(z)=\frac{G_{q_{2}}(1)}{\Gamma(-1 / 4)} \frac{Z}{(\log Z)^{5 / 4}}\left\{1+O\left(\frac{q_{2}^{\varepsilon}}{\log Z}\right)\right\} .
$$

Noting that $\Gamma(-1 / 4)<0$, an application of partial summation completes the proof.

7.2. Asymptotic formula for $N_{\mathrm{Br}}(P)$. - Our starting point in the proof of Theorem 1.3 is (5.2), followed by the changes of variables (5.4), (5.5) and (6.4). In the present situation a further change of variables will be expedient. Define

$$
\begin{array}{ll}
\ell_{1}^{\prime \prime}=\operatorname{gcd}\left(a^{\prime \prime \prime},\left(m^{\prime \prime} n^{\prime \prime}\right)^{\infty}\right), & \ell_{2}^{\prime \prime}=\operatorname{gcd}\left(b^{\prime \prime \prime},\left(m^{\prime \prime} n^{\prime \prime}\right)^{\infty}\right), \\
\ell_{3}^{\prime \prime}=\operatorname{gcd}\left(c^{\prime \prime \prime},\left(m^{\prime \prime} n^{\prime \prime}\right)^{\infty}\right), & \ell_{4}^{\prime \prime}=\operatorname{gcd}\left(d^{\prime \prime \prime},\left(m^{\prime \prime} n^{\prime \prime}\right)^{\infty}\right) .
\end{array}
$$

We now write

$$
a^{\prime \prime \prime}=\ell_{1}^{\prime \prime} \tilde{a}, \quad b^{\prime \prime \prime}=\ell_{2}^{\prime \prime} \tilde{b}, \quad c^{\prime \prime \prime}=\ell_{3}^{\prime \prime} \tilde{c}, \quad d^{\prime \prime \prime}=\ell_{4}^{\prime \prime} \tilde{d}
$$


for $\tilde{a}, \tilde{b}, \tilde{c}, \tilde{d} \in \mathbb{N}$ satisfying $\operatorname{gcd}\left(\tilde{a} \tilde{b} \tilde{c} \tilde{d}, m^{\prime \prime} n^{\prime \prime}\right)=1$. The union of this constraint with (6.5) and (6.6) is equivalent to

$$
\operatorname{gcd}\left(\tilde{a} \tilde{b} \tilde{c} \tilde{d}, 2 m n m^{\prime \prime} n^{\prime \prime}\right)=1
$$

and

$$
\operatorname{gcd}(\tilde{a} \tilde{d}, \tilde{b} \tilde{c})=1
$$

with $\ell_{1}^{\prime \prime}, \ldots, \ell_{4}^{\prime \prime} \in \mathbb{N}$ constrained to satisfy

$$
\operatorname{gcd}\left(\ell_{2}^{\prime \prime} \ell_{4}^{\prime \prime}, m^{\prime \prime}\right)=\operatorname{gcd}\left(\ell_{1}^{\prime \prime} \ell_{3}^{\prime \prime}, n^{\prime \prime}\right)=1, \quad \operatorname{gcd}\left(\ell_{i}^{\prime \prime}, \ell_{j}^{\prime \prime}\right)=1,
$$

for $1 \leqslant i<j \leqslant 4$. Note that since $\ell_{i}^{\prime \prime} \mid\left(m^{\prime \prime} n^{\prime \prime}\right)^{\infty}$ it automatically follows that $\ell_{i}^{\prime \prime}$ is coprime to $2 m n$. In what follows it will be convenient to redefine

$$
\begin{aligned}
& A=m m^{\prime \prime} \ell_{1} \ell_{1}^{\prime \prime}, \quad B=m n^{\prime \prime} \ell_{2} \ell_{2}^{\prime \prime} 2^{\beta}, \\
& C=n m^{\prime \prime} \ell_{3} \ell_{3}^{\prime \prime} 2^{\gamma}, \quad D=n n^{\prime \prime} \ell_{4} \ell_{4}^{\prime \prime} 2^{\delta} .
\end{aligned}
$$

Under our various transformations we may now write $\Delta^{\prime}=m^{\prime \prime} n^{\prime \prime} \Delta^{\prime \prime}$, with

$$
\Delta^{\prime \prime}=\varepsilon_{4} \ell_{1} \ell_{1}^{\prime \prime} \ell_{4} \ell_{4}^{\prime \prime} 2^{\delta} \tilde{a} \tilde{d}-\varepsilon_{2} \varepsilon_{3} \ell_{2} \ell_{2}^{\prime \prime} \ell_{3} \ell_{3}^{\prime \prime} 2^{\beta+\gamma} \tilde{b} \tilde{c} .
$$

In this new notation we must proceed to consider the constraints recorded in $\$ 4$ which are both necessary and sufficient to have $X_{a, b, c, d}\left(\mathbb{Q}_{v}\right) \neq \emptyset$ for all $v \in \Omega$, but $X_{a, b, c, d}(\mathbb{Q})=\emptyset$. Our key tool is Lemma 4.1 and the associated calculations.

For the infinite valuation we must have $\varepsilon=\left(\varepsilon_{2}, \varepsilon_{3}, \varepsilon_{4}\right) \notin\{(+,-,-),(-,+,-)\}$ by part (i) of Lemma 3.1 and Lemma 4.5. For the primes $p \equiv 1(\bmod 4)$ there are no additional constraints arising. The situation is more complicated for the primes $p \equiv 3(\bmod 4)$. According to Lemma 4.6 we seek constraints under which there exists a unique choice of $\mathbf{k} \in\{0,1\}^{2}$ with $k_{1}+k_{2} \equiv v_{p}(m n)(\bmod 2)$ and $W_{\left(p^{\left.k_{1}, p^{k_{2}}\right)}\right.}\left(\mathbb{Q}_{p}\right) \neq \emptyset$, where $W_{\mathbf{e}}$ is given in Lemma 4.1.

Suppose that $p \mid m n$, so that $v_{p}(m n)=1$. Then Lemma 3.1(iii) implies that (6.7) doesn't hold, that $p \nmid a^{\prime \prime} b^{\prime \prime} c^{\prime \prime} d^{\prime \prime}$ and that

$$
\left(\frac{-\varepsilon_{2} \ell_{1, p} \ell_{2, p} a^{\prime \prime} b^{\prime \prime}}{p}\right)+\left(\frac{-\varepsilon_{3} \varepsilon_{4} \ell_{3, p} \ell_{4, p} c^{\prime \prime} d^{\prime \prime}}{p}\right) \geqslant 0,
$$

if $v_{p}\left(\ell_{i}\right) \in 2 \mathbb{Z}$ and $\ell_{i, p}=p^{-v_{p}\left(\ell_{i}\right)} \ell_{i}$, for $1 \leqslant i \leqslant 4$. The constraint in Lemma 4.3(iii) gives either $v_{p}\left(\ell_{i}\right) \in 2 \mathbb{N}$ and $v_{p}\left(\ell_{i+2}\right)=1$ for $i \in\{1,2\}$, or $v_{p}\left(\ell_{i}\right)=1$ and $v_{p}\left(\ell_{i+2}\right) \in 2 \mathbb{N}$ for $i \in\{1,2\}$. The constraint in Lemma 4.3(i) translates as

$$
\left[\frac{-\varepsilon_{2} \ell_{1} \ell_{2} a^{\prime \prime} b^{\prime \prime}}{p}\right]+\left[\frac{-\varepsilon_{3} \varepsilon_{4} \ell_{3} \ell_{4} c^{\prime \prime} d^{\prime \prime}}{p}\right] \leqslant 0 .
$$

It follows from (5.7) that $v_{p}\left(\ell_{1} \ell_{2}\right)$ and $v_{p}\left(\ell_{3} \ell_{4}\right)$ cannot both be odd. On recalling from (5.6) that $\ell_{1}, \ell_{2}$ are coprime, we see that $v_{p}\left(\ell_{1}\right) \in 2 \mathbb{Z}$ if and only if $v_{p}\left(\ell_{1} \ell_{2}\right) \in 2 \mathbb{Z}$. Similarly, $v_{p}\left(\ell_{3}\right) \in 2 \mathbb{Z}$ if and only if $v_{p}\left(\ell_{3} \ell_{4}\right) \in 2 \mathbb{Z}$, since $\ell_{3}, \ell_{4}$ are coprime. Combining all these conditions therefore leads to the description that $p \nmid a^{\prime \prime} b^{\prime \prime} c^{\prime \prime} d^{\prime \prime}$, with one of the following

$-v_{p}\left(\ell_{i}\right) \in 2 \mathbb{N}$ and $v_{p}\left(\ell_{i+2}\right)=1$ for $i \in\{1,2\}$

$-v_{p}\left(\ell_{i}\right)=1$ and $v_{p}\left(\ell_{i+2}\right) \in 2 \mathbb{N}$ for $i \in\{1,2\}$;

$-v_{p}\left(\ell_{1} \ell_{2}\right) \in 2 \mathbb{Z}$ and $v_{p}\left(\ell_{3} \ell_{4}\right) \in 2 \mathbb{Z}$, with

$$
\left(\frac{-\varepsilon_{2} \ell_{1, p} \ell_{2, p} a^{\prime \prime} b^{\prime \prime}}{p}\right)+\left(\frac{-\varepsilon_{3} \varepsilon_{4} \ell_{3, p} \ell_{4, p} c^{\prime \prime} d^{\prime \prime}}{p}\right)=0
$$


- $v_{p}\left(\ell_{1} \ell_{2}\right) \in 2 \mathbb{Z}$ and $v_{p}\left(\ell_{3} \ell_{4}\right) \notin 2 \mathbb{Z}$, with

$$
\left(\frac{-\varepsilon_{2} \ell_{1, p} \ell_{2, p} a^{\prime \prime} b^{\prime \prime}}{p}\right)=-1
$$

$-v_{p}\left(\ell_{1} \ell_{2}\right) \notin 2 \mathbb{Z}$ and $v_{p}\left(\ell_{3} \ell_{4}\right) \in 2 \mathbb{Z}$, with

$$
\left(\frac{-\varepsilon_{3} \varepsilon_{4} \ell_{3, p} \ell_{4, p} c^{\prime \prime} d^{\prime \prime}}{p}\right)=-1
$$

For given parameters $\varepsilon, m, n, m^{\prime \prime}, n^{\prime \prime}, \ell, \ell^{\prime \prime}$, the constraints arising from primes $p \equiv 3(\bmod 4)$ for which $p \mid m n$ force the vector $(\tilde{a}, \tilde{b}, \tilde{c}, \tilde{d})$ to lie in one of finitely many congruence classes modulo $p$. Let us denote the set of possible classes by

$$
\tilde{T}_{m n}(\boldsymbol{\ell}) \cong \bigoplus_{p \mid m n} \tilde{T}_{p}(\boldsymbol{\ell})
$$

Here $\tilde{T}_{m n}(\ell) \subseteq(\mathbb{Z} / m n \mathbb{Z})^{4}$ and $\tilde{T}_{p}(\ell) \subseteq(\mathbb{Z} / p \mathbb{Z})^{4}$ for each prime $p$. Although $\tilde{T}_{m n}(\ell)$ also depends on $\varepsilon, m^{\prime \prime}, n^{\prime \prime}$ and $\ell^{\prime \prime}$, its cardinality does not, as the following result shows.

Lemma 7.3. - Let $p \mid m n$. Then we have

$$
\# \tilde{T}_{p}(\ell)=\frac{1}{2^{1-\tau\left(v_{p}\left(\ell_{1}\right), \ldots, v_{p}\left(\ell_{4}\right)\right)}}(p-1)^{4},
$$

where for $\boldsymbol{\nu} \in \mathbb{Z}_{\geqslant 0}^{4}$ we define

$$
\tau(\boldsymbol{\nu})= \begin{cases}1, & \text { if } \nu_{i} \in 2 \mathbb{N} \text { and } \nu_{i+2}=1 \text { for } i \in\{1,2\} \\ 1, & \text { if } \nu_{i}=1 \text { and } \nu_{i+2} \in 2 \mathbb{N} \text { for } i \in\{1,2\} \\ 0, & \text { otherwise }\end{cases}
$$

Proof. - This follows since the Legendre symbols $\left(\frac{-\varepsilon_{2} \ell_{1, p} \ell_{2, p} a^{\prime \prime} b^{\prime \prime}}{p}\right)$ and $\left(\frac{-\varepsilon_{3} \varepsilon_{4} \ell_{3, p} \ell_{4, p} c^{\prime \prime} d^{\prime \prime}}{p}\right)$ only take two possible values, whatever the parity of $v_{p}\left(\ell_{1} \ell_{2}\right)$ or $v_{p}\left(\ell_{3} \ell_{4}\right)$.

Next we suppose that $p \nmid m n m^{\prime \prime} n^{\prime \prime}$, still with $p \equiv 3(\bmod 4)$. Recall that $\Delta^{\prime}=m^{\prime \prime} n^{\prime \prime} \Delta^{\prime \prime}$, with $\Delta^{\prime \prime}$ given by (7.9). Then according to Lemma 4.3(ii) we must avoid the the case

$$
\left(\frac{-\varepsilon_{2} a^{\prime} b^{\prime}}{p}\right)=\left(\frac{-\varepsilon_{3} \varepsilon_{4} c^{\prime} d^{\prime}}{p}\right)=1
$$

if $p \mid \Delta^{\prime}$ and $p \nmid a^{\prime} b^{\prime} c^{\prime} d^{\prime}$. Note that $p \mid \Delta^{\prime}$ and $p \nmid a^{\prime} b^{\prime} c^{\prime} d^{\prime}$ if and only if $p \mid \Delta^{\prime \prime}$ and $p \nmid m^{\prime \prime} n^{\prime \prime}$. But for such primes $\left(\frac{-\varepsilon_{2} a^{\prime} b^{\prime}}{p}\right)$ and $\left(\frac{-\varepsilon_{2} \varepsilon_{3} c^{\prime} d^{\prime}}{p}\right)$ are equal. Hence, since $p \equiv 3(\bmod 4)$, we see that the constraint is equivalent to

$$
\left(\frac{\varepsilon_{2} a^{\prime} b^{\prime}}{p}\right)=1
$$

if $p \mid \Delta^{\prime \prime}$.

Suppose now that $p \nmid m n$ and $p \mid m^{\prime \prime} n^{\prime \prime}$. In particular $p \mid \Delta^{\prime}$ and Lemma 4.3 implies that we must have $\left[\frac{-\varepsilon_{2} a^{\prime} b^{\prime}}{p}\right]+\left[\frac{-\varepsilon_{3} \varepsilon_{4} c^{\prime} d^{\prime}}{p}\right] \leqslant 0$. We cannot have both $v_{p}\left(a^{\prime} b^{\prime}\right)$ and $v_{p}\left(c^{\prime} d^{\prime}\right)$ being odd. Moreover, we must have $\min \left\{v_{p}\left(a^{\prime} b^{\prime}\right), v_{p}\left(c^{\prime} d^{\prime}\right)\right\} \leqslant 1$. We deduce that precisely one of $v_{p}\left(\ell_{1}^{\prime \prime} \ell_{2}^{\prime \prime}\right)$ or $v_{p}\left(\ell_{3}^{\prime \prime} \ell_{4}^{\prime \prime}\right)$ is odd. Hence we have the pair of conditions

$$
\left(v_{p}\left(\ell_{1}^{\prime \prime} \ell_{2}^{\prime \prime}\right), v_{p}\left(\ell_{3}^{\prime \prime} \ell_{4}^{\prime \prime}\right)\right)=(0,1)(\bmod 2), \quad\left[\frac{-\varepsilon_{2} a^{\prime} b^{\prime}}{p}\right]=-1,
$$


or

$$
\left(v_{p}\left(\ell_{1}^{\prime \prime} \ell_{2}^{\prime \prime}\right), v_{p}\left(\ell_{3}^{\prime \prime} \ell_{4}^{\prime \prime}\right)\right)=(1,0)(\bmod 2), \quad\left[\frac{-\varepsilon_{3} \varepsilon_{4} c^{\prime} d^{\prime}}{p}\right]=-1 .
$$

In accordance with this we are now led to introduce the set

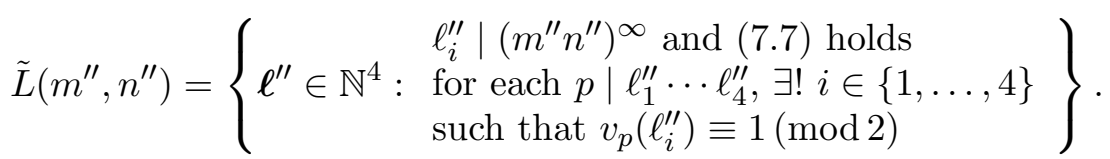

For given parameters $\varepsilon, m, n, m^{\prime \prime}, n^{\prime \prime}, \ell, \ell^{\prime \prime}$, with $\ell^{\prime \prime} \in \tilde{L}\left(m^{\prime \prime}, n^{\prime \prime}\right)$, the constraints arising from primes $p \equiv 3(\bmod 4)$ for which $p \mid m^{\prime \prime} n^{\prime \prime}$ force the vector $(\tilde{a}, \tilde{b}, \tilde{c}, \tilde{d})$ to lie in one of finitely many congruence classes modulo $p$. Let us denote the set of possible classes by

$$
\tilde{U}_{m^{\prime \prime} n^{\prime \prime}}\left(\ell^{\prime \prime}\right) \cong \bigoplus_{p \mid m^{\prime \prime} n^{\prime \prime}} \tilde{U}_{p}\left(\ell^{\prime \prime}\right)
$$

The analogue of Lemma 7.3 is the following easy result.

Lemma 7.4. - Let $p \mid m^{\prime \prime} n^{\prime \prime}$. Then we have $\# \tilde{U}_{p}\left(\ell^{\prime \prime}\right)=\frac{1}{2}(p-1)^{4}$.

For any $(a, b, c, d)$ constrained as above, our work so far has shown that there exists a unique e $\in \mathscr{B}^{2}$ satisfying (4.1) for some $f \in \mathscr{B}$ with $\operatorname{gcd}(f, m n)=1$, such that $W_{\mathbf{e}}\left(\mathbb{Q}_{v}\right) \neq \emptyset$ for every valuation $v \neq 2$. Suppose that

$$
e_{i} \equiv \epsilon_{i}(\bmod 4), \quad(i=1,2),
$$

for $\epsilon_{1}, \epsilon_{2} \in\{ \pm 1\}$. For the prime $p=2$, it remains to distinguish precisely when $X_{a, b, c, d}\left(\mathbb{Q}_{2}\right)$ is non-empty but $W_{\mathbf{e}}\left(\mathbb{Q}_{2}\right)$ is empty. This is equivalent to demanding that

$$
\left(\epsilon_{1} a^{\prime}, \epsilon_{1} \varepsilon_{2} b^{\prime}, \epsilon_{2} \varepsilon_{3} c^{\prime}, \epsilon_{2} \varepsilon_{4} d^{\prime}\right) \in T_{\text {tot }}^{(2)} \backslash T_{\text {tot }}^{(1)},
$$

in the notation of $\$ 4.4$. An inspection of Lemmas 4.8 4.15 shows that for a given choice of $(\beta, \gamma, \delta) \in L_{2}^{(\iota)}$, the 2-adic constraints placed on $(\tilde{a}, \tilde{b}, \tilde{c}, \tilde{d})$ take the shape of a union of particular congruence classes modulo 16 . Let us denote by $\tilde{H}_{\beta, \gamma, \delta}$ the set of all possible classes modulo 16 that can arise. Then the pair of constraints $X_{a, b, c, d}\left(\mathbb{Q}_{2}\right) \neq \emptyset$ and $W_{\mathbf{e}}\left(\mathbb{Q}_{2}\right)=\emptyset$ are equivalent to demanding that

$$
(\tilde{a}, \tilde{b}, \tilde{c}, \tilde{d}) \in \tilde{H}_{\beta, \gamma, \delta}(\bmod 16)
$$

for given $(\beta, \gamma, \delta) \in L_{2}^{(\iota)}$. In particular this constraint implies that $\tilde{a} \tilde{b} \tilde{c} \tilde{d}$ is odd. Although $\tilde{H}_{\beta, \gamma, \delta}$ depends on numerous parameters, including the residue class of e modulo 4 , its cardinality is independent of all of these. We will set

$$
\sigma_{2}=\sum_{\iota \in\{0,1\}} \sum_{(\beta, \gamma, \delta) \in L_{2}^{(\iota)}} \frac{\# \tilde{H}_{\beta, \gamma, \delta}}{2^{16+\beta+\gamma+\delta}}
$$

This constant is equal to the density of points $(a, b, c, d)$ for which there exist coprime integers $u, v \in \mathbb{Z}_{2}$ for which $Q_{1} Q_{2}(u, v) \in \mathscr{D}$, yet for every choice of coprime $u, v \in \mathbb{Z}_{2}$ one never has both $Q_{1}(u, v) \in \mathscr{D}$ and $Q_{2}(u, v) \in \mathscr{D}$. Using our work in $₫ 4.4$, as an analogue of Lemma 6.2, it is in principle possible to calculate a numerical value for $\sigma_{2}$. Such a calculation would be both lengthy and tedious, and we have chosen not to pursue this here. For our purposes it will be sufficient to note that $\sigma_{2}>0$, since $T_{\text {tot }}^{(1)} \neq T_{\text {tot }}^{(2)}$. 
We are now ready to return to the expression (5.2) for $N_{\mathrm{Br}}(P)$. It will be convenient to put

$$
M=16 m n m^{\prime \prime} n^{\prime \prime} .
$$

Let us define the function

$$
h\left(\Delta^{\prime \prime}, \tilde{a}, \tilde{b}, M\right)=\prod_{\substack{p \mid \Delta^{\prime \prime} \\ p \nmid M \\ p \equiv 3(\bmod 4)}} \frac{1}{2}\left\{1+\left(\frac{\varepsilon_{2} A B \tilde{a} \tilde{b}}{p}\right)\right\},
$$

where $A, B$ are given by (7.8) . One notes that $0 \leqslant h\left(\Delta^{\prime \prime}, \tilde{a}, \tilde{b}, M\right) \leqslant 1$ and

$$
h\left(\Delta^{\prime \prime}, \tilde{a}, \tilde{b}, M\right)=\sum_{z \mid \Delta^{\prime \prime}} g_{\mathbf{a}, \mathbf{q}}(z),
$$

in the notation of (17.1), with $\mathbf{a}=(\tilde{a}, \tilde{b})$ and $\mathbf{q}=\left(\varepsilon_{2} A B, M\right)$. In particular $g_{\mathbf{a}, \mathbf{q}}$ is only supported on positive integers coprime to $\tilde{a} \tilde{b} \tilde{c} \tilde{d} M$, which are built from primes congruent to 3 modulo 4. Summarising our investigation so far, our analogue of (6.8) is

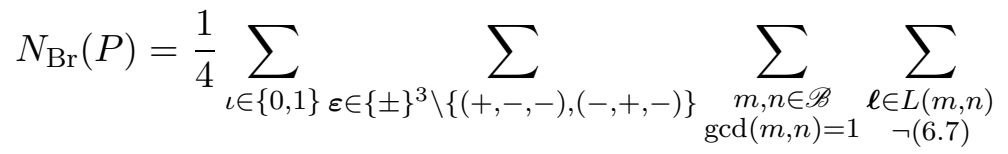

$$
\begin{aligned}
& \times \sum_{\substack{m^{\prime \prime}, n^{\prime \prime} \in \mathscr{A} \\
\operatorname{gcd}\left(m^{\prime \prime}, n^{\prime \prime}\right)=1 \\
\operatorname{gcd}\left(m^{\prime \prime} n^{\prime \prime}, 2 m n\right)=1}} \sum_{(\beta, \gamma, \delta) \in L_{2}^{(\iota)}} \sum_{\ell^{\prime \prime} \in \tilde{L}\left(m^{\prime \prime}, n^{\prime \prime}\right)} \tilde{N}+O\left(P^{2+\varepsilon}\right),
\end{aligned}
$$

where $L(m, n)\left(\operatorname{resp} . L_{2}^{(\iota)}, \tilde{L}\left(m^{\prime \prime}, n^{\prime \prime}\right)\right)$ is given by (5.8) (resp. (6.3), (7.10) $)$ and

$$
\tilde{N}=\sum_{\tilde{t}=(\tilde{a}, \tilde{b}, \tilde{c}, \tilde{d})} h\left(\Delta^{\prime \prime}, \tilde{a}, \tilde{b}, M\right) .
$$

The conditions of summation here are restricted to $\tilde{t} \in \mathbb{N}^{4}$ such that (7.5), (7.6) and (7.11) hold, with $A \tilde{a}, B \tilde{b}, C \tilde{c}, D \tilde{d} \leqslant P$ and $\tilde{t} \in \tilde{T}_{m n}(\ell)(\bmod m n) \cap \tilde{U}_{m^{\prime \prime} n^{\prime \prime}}\left(\ell^{\prime \prime}\right)\left(\bmod m^{\prime \prime} n^{\prime \prime}\right)$. The definitions of $\tilde{T}_{m n}(\ell)$ and $\tilde{U}_{m^{\prime \prime} n^{\prime \prime}}\left(\ell^{\prime \prime}\right)$ ensure that the product $\tilde{a} \tilde{b} \tilde{c} \tilde{d}$ is coprime to $m n m^{\prime \prime} n^{\prime \prime}$. Likewise (7.11) implies that $\tilde{a} \tilde{b} \tilde{c} \tilde{d}$ is odd. Hence (7.5) is redundant.

In our analysis of (7.14) it will frequently be useful to reduce the allowable ranges for the various parameters appearing in the outer summations. Two quantities that will feature heavily in this process are

$$
T_{1}=(\log P)^{2}, \quad T_{2}=(\log P)^{100} .
$$

Recall the definitions (7.8) of $A, B, C, D$. We proceed to establish the following result.

Lemma 7.5. - Let $\varepsilon>0$ and $\boldsymbol{\delta} \in\{0,1\}^{3}$. For $1 \leqslant R_{1} \leqslant R_{2}$, we have

$$
\sum_{m, n} \sum_{\ell} \sum_{m^{\prime \prime}, n^{\prime \prime}} \sum_{\beta, \gamma, \delta} \sum_{\ell^{\prime \prime}} \frac{1}{(A B)^{\delta_{1}}(C D)^{\delta_{2}} M^{\delta_{3}}} \ll \begin{cases}R_{2}^{4+\varepsilon}, & \text { if } \boldsymbol{\delta}=\mathbf{0}, \\ R_{1}^{-1+\varepsilon}, & \text { if } \boldsymbol{\delta}=(1,1,0), \\ R_{2}^{\varepsilon,}, & \text { if } \boldsymbol{\delta}=(1,0,1),\end{cases}
$$

uniformly in $R_{1}, R_{2}$, where the sum is subject to

$$
R_{1} \leqslant \max \left\{m, n, m^{\prime \prime}, n^{\prime \prime}, \ell_{i}, \ell_{i}^{\prime \prime}, 2^{\beta}, 2^{\gamma}, 2^{\delta}\right\} \leqslant R_{2} .
$$


Proof. - Let us denote the expression that is to be estimate by $\Sigma_{\boldsymbol{\delta}}$. Suppose first that $\boldsymbol{\delta}=\mathbf{0}$. Then there are $\log \left(R_{2}+1\right)^{3}$ choices for $\beta, \gamma, \delta$. Recall the definition (2.10) of $\varphi_{\delta}^{*}$, for any $\delta>0$. Rankin's trick and (5.15) allow us to deduce that

$$
\begin{aligned}
\Sigma_{\mathbf{0}} & \ll \log \left(R_{2}+1\right)^{3} \sum_{m, n} \sum_{\ell} \frac{R_{2}^{\varepsilon / 4}}{\left(\ell_{1} \cdots \ell_{4}\right)^{\varepsilon / 16}} \sum_{m^{\prime \prime}, n^{\prime \prime}} \sum_{\ell^{\prime \prime}} \frac{R_{2}^{\varepsilon / 4}}{\left(\ell_{1}^{\prime \prime} \cdots \ell_{4}^{\prime \prime}\right)^{\varepsilon / 16}} \\
& \ll R_{2}^{\varepsilon / 2} \log \left(R_{2}+1\right)^{3}\left(\sum_{k \leqslant R_{2}} \frac{1}{\varphi_{\varepsilon / 16}^{*}(k)^{4}}\right)^{4} \\
& \ll R_{2}^{4+\varepsilon},
\end{aligned}
$$

as required.

Let us next consider the case $\boldsymbol{\delta}=(1,1,0)$. Then our argument in 6 , which was used to restrict the size of the parameters appearing in (6.8), easily gives $\Sigma_{(1,1,0)}=O\left(R_{1}^{-1+\varepsilon}\right)$, again using Rankin's trick and (5.15) to handle the sum over $\ell$ and $\ell^{\prime \prime}$. Finally we must consider the case $\boldsymbol{\delta}=(1,0,1)$. In this case, since $2^{\beta}, 2^{\gamma}, 2^{\delta} \leqslant R_{2}$ and the sum over $\beta$ is absolutely convergent, we obtain

$$
\Sigma_{(1,0,1)} \ll \log \left(R_{2}+1\right)^{2} \sum_{m, n, m^{\prime \prime}, n^{\prime \prime}} \frac{1}{m^{3} n\left(m^{\prime \prime} n^{\prime \prime}\right)^{2}} \sum_{\ell, \ell^{\prime \prime}} \frac{1}{\ell_{1} \ell_{2} \ell_{1}^{\prime \prime} \ell_{2}^{\prime \prime}} .
$$

Using Rankin's trick again, we see that

$$
\sum_{\ell, \ell^{\prime \prime}} \frac{1}{\ell_{1} \ell_{2} \ell_{1}^{\prime \prime} \ell_{2}^{\prime \prime}} \leqslant \frac{R_{2}^{\varepsilon / 2}}{\varphi_{1+\varepsilon / 8}^{*}(m n)^{2} \varphi_{1+\varepsilon / 8}^{*}\left(m^{\prime \prime} n^{\prime \prime}\right)^{2}} \ll R_{2}^{\varepsilon / 2},
$$

since $1 / \varphi_{\delta}^{*}(n) \ll \delta 1$ for $\delta>1$. Hence $\Sigma_{(1,0,1)} \ll R_{2}^{\varepsilon / 2} \log \left(R_{2}+1\right)^{3} \ll R_{2}^{\varepsilon}$, as required.

A trivial upper bound is given by $\tilde{N} \ll P^{4} /(A B C D)$. Applying Lemma 7.5 with $\boldsymbol{\delta}=(1,1,0)$ and $R_{1}=T_{1}=(\log P)^{2}$, we may henceforth restrict attention to parameters in (7.14) for which

$$
\max \left\{m, n, m^{\prime \prime}, n^{\prime \prime}, \ell_{i}, \ell_{i}^{\prime \prime}, 2^{\beta}, 2^{\gamma}, 2^{\delta}\right\} \leqslant T_{1},
$$

with satisfactory overall error $O\left(P^{4}(\log P)^{-2+\varepsilon}\right)$.

We wish to break the sum into congruence classes modulo $M$. We shall introduce the set $T_{M}$, say, of $t_{0}=\left(a_{0}, b_{0}, c_{0}, d_{0}\right)(\bmod M)$ for which

$$
t_{0} \in \tilde{T}_{m n}(\ell)(\bmod m n) \cap \tilde{U}_{m^{\prime \prime} n^{\prime \prime}}\left(\ell^{\prime \prime}\right)\left(\bmod m^{\prime \prime} n^{\prime \prime}\right) \cap \tilde{H}_{\beta, \gamma, \delta}(\bmod 16) .
$$

These two conditions imply that $\operatorname{gcd}\left(a_{0} b_{0} c_{0} d_{0}, M\right)=1$. We may therefore write

$$
\tilde{N}=\sum_{t_{0} \in T_{M}} \sum_{\substack{\tilde{a} \leqslant P / A, \tilde{b} \leqslant P / B \\(\tilde{a}, \tilde{b}) \equiv\left(a_{0}, b_{0}\right)(\bmod M)}} \sum_{\substack{\tilde{c} \leqslant P / C, \tilde{d} \leqslant P / D \\ \operatorname{gcd}(\tilde{a} \tilde{d} \tilde{b} \tilde{c})=1 \\(\tilde{c}, \tilde{d}) \equiv\left(c_{0}, d_{0}\right)(\bmod M)}} h\left(\Delta^{\prime \prime}, \tilde{a}, \tilde{b}, M\right) .
$$

Note from (7.9) that $\Delta^{\prime \prime} \ll P^{2} / M$. Invoking (7.13) we obtain a sum over $z$ which a priori runs over all integers up to order $P^{2} / M$. Define the 2-dimensional lattice

$$
\Lambda_{z}=\left\{(X, Y) \in \mathbb{Z}^{2}: \varepsilon_{4} \ell_{1} \ell_{1}^{\prime \prime} \ell_{4} \ell_{4}^{\prime \prime} 2^{\delta} \tilde{a} Y-\varepsilon_{2} \varepsilon_{3} \ell_{2} \ell_{2}^{\prime \prime} \ell_{3} \ell_{3}^{\prime \prime} 2^{\beta+\gamma} \tilde{b} X \equiv 0(\bmod z)\right\} .
$$


We then have

$$
\tilde{N}=\sum_{t_{0} \in T_{M}} \sum_{\substack{\tilde{a} \leqslant P / A, \tilde{b} \leqslant P / B \\(\tilde{a}, \tilde{b}) \equiv\left(a_{0}, b_{0}\right)(\bmod M)}} \sum_{\substack{z \ll P^{2} / M \\ \operatorname{gcd}(z, \tilde{a} \tilde{b} M)=1}} g_{\mathbf{a}, \mathbf{q}}(z) \# \mathscr{A}_{z},
$$

where

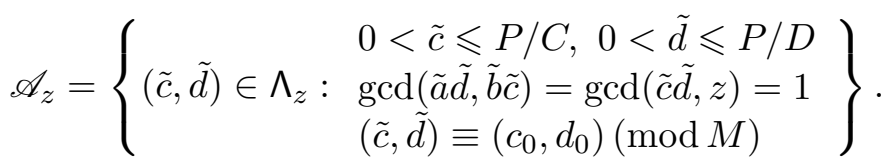

It will be crucial to show that there is a negligible contribution from large values of $z$ in this sum. This will be achieved using Lemma 7.1.

According to Heath-Brown [5, Lemma 2] we have

$$
\sum_{c_{0}, d_{0}} \# \mathscr{A}_{z} \ll \frac{P^{2}}{C D z}+1
$$

Recall the definition (7.15) of $T_{2}$. The overall contribution to $\tilde{N}$ from $z>P^{2} /\left(M T_{2}\right)$ is therefore seen to be

$$
\begin{aligned}
& \ll \sum_{a_{0}, b_{0}} \sum_{\substack{a \leqslant P / A, \tilde{b} \leqslant P / B \\
(\tilde{a}, \tilde{b}) \equiv\left(a_{0}, b_{0}\right)(\bmod M)}} \sum_{P^{2} /\left(M T_{2}\right)<z \ll P^{2} / M}\left|g_{\mathbf{a}, \mathbf{q}}(z)\right|\left(\frac{P^{2}}{C D z}+1\right) \\
& \leqslant \sum_{\substack{\tilde{a} \leqslant P / A, \tilde{b} \leqslant P / B \\
P^{2} /\left(M T_{2}\right)<z \ll P^{2} / M}}\left|g_{\mathbf{a}, \mathbf{q}}(z)\right|\left(\frac{P^{2}}{C D z}+1\right) .
\end{aligned}
$$

Note here that $P^{2} /\left(M T_{2}\right) \gg P^{2} /\left(T_{1}^{4} T_{2}\right) \gg P^{2} /(\log P)^{108}$. We break the summation over $z$ into $O\left(\log T_{2}\right)$ dyadic intervals $Z / 2 \leqslant z \leqslant Z$. Lemma 7.1 therefore yields the contribution

$$
\begin{aligned}
& \ll\left(\log T_{2}\right) \max _{P^{2} /\left(M T_{2}\right) \ll Z \ll P^{2} / M}\left(\frac{P^{2}}{C D}+Z\right)\left(\frac{P^{2}}{A B(\log Z)^{3 / 4}}+\sqrt{\frac{P^{2}}{A B}} Z^{3 / 8+\varepsilon}\right) \\
& \ll \frac{P^{4} \log \log P}{A B(\log P)^{3 / 4}}\left(\frac{1}{C D}+\frac{1}{M}\right)+P^{4-1 / 4+\varepsilon} .
\end{aligned}
$$

It remains to sum this over the remaining parameters subject to (7.16). Taking $R_{1}=1$ and $R_{2}=T_{1}$ in Lemma 7.5, one arrives at the overall contribution $O\left(P^{4}(\log P)^{-3 / 4+\varepsilon}\right)$, which is satisfactory for Theorem 1.3 .

We may now focus our attention on the contribution from $z \leqslant P^{2} /\left(M T_{2}\right)$ in (7.18), which we denote by $\tilde{N}_{1}$. We will need to take care of the coprimality conditions $\operatorname{gcd}(\tilde{a} \tilde{d}, \tilde{b} \tilde{c})=1$ in $\mathscr{A}_{z}$, retaining the condition $\operatorname{gcd}(\tilde{c} \tilde{d}, z)=1$. Recall that modulo $M$ the first condition is already implied by the definition of $T_{M}$, as is the fact that $\operatorname{gcd}(\tilde{a} \tilde{b}, M)=1$. In this way we 
deduce that

$$
\begin{gathered}
\tilde{N}_{1}=\sum_{t_{0} \in T_{M}} \sum_{\substack{\tilde{a} \leqslant P / A, \tilde{b} \leqslant P / B \\
\operatorname{gcd}(\tilde{a}, \tilde{b})=1 \\
(\tilde{a}, \tilde{b}) \equiv\left(a_{0}, b_{0}\right)(\bmod M)}} \sum_{\substack{z \leqslant P^{2} /\left(M T_{2}\right) \\
\operatorname{gcd}(z, \tilde{a} \tilde{b} M)=1}} g_{\mathbf{a}, \mathbf{q}}(z) \\
\times \sum_{\substack{k_{1} \leqslant P \\
\operatorname{gcd}\left(k_{1}, M z\right)=1}} \sum_{\substack{k_{2}\left|\tilde{a} \\
k_{3}\right| \tilde{b}}} \mu\left(k_{1}\right) \mu\left(k_{2}\right) \mu\left(k_{3}\right) \# \mathscr{A}_{z, \mathbf{k}},
\end{gathered}
$$

where a change of variables yields

$$
\mathscr{A}_{z, \mathbf{k}}=\left\{\begin{array}{ll} 
& \left(\left[k_{1}, k_{2}\right] X,\left[k_{1}, k_{3}\right] Y\right) \in \Lambda_{z} \\
(X, Y) \in \mathbb{N}^{2}: & X \leqslant P /\left(\left[k_{1}, k_{2}\right] C\right), Y \leqslant P /\left(\left[k_{1}, k_{3}\right] D\right) \\
& (X, Y) \equiv\left(X_{0}, Y_{0}\right)(\bmod M) \\
& \operatorname{gcd}(X Y, z)=1
\end{array}\right\} .
$$

Here, if $\overline{\left[k_{1}, k_{j}\right]} \in \mathbb{Z}$ denotes the multiplicative inverse of $\left[k_{1}, k_{j}\right]$ modulo $M$ for $j=2,3$, then $X_{0}=\overline{\left[k_{1}, k_{2}\right]} c_{0}$ and $Y_{0}=\overline{\left[k_{1}, k_{3}\right]} d_{0}$. Finally, we note that $k_{2} k_{3}$ is automatically coprime to $M z$, since $\tilde{a} \tilde{b}$ satisfies this property.

We will also need to reduce the ranges of summation for $k_{1}, k_{2}, k_{3}$ in this expression. Recall from (17.1) that $\left|g_{\mathbf{a}, \mathbf{q}}(z)\right| \leqslant 1$ for any $z$. To achieve our goal we invert the summation over $z$ and $(X, Y)$, finding that the overall contribution to $\tilde{N}_{1}$ from $k_{1}>\sqrt{T_{2}}$ is

$$
\ll \sum_{t_{0} \in T_{M}} \sum_{\substack{\tilde{a} \leqslant P / A, \tilde{b} \leqslant P / B \\ \operatorname{gcd}(\tilde{a}, \tilde{b})=1 \\(\tilde{a}, \tilde{b}) \equiv\left(a_{0}, b_{0}\right)(\bmod M)}} \sum_{\substack{\sqrt{T_{2}}<k_{1} \leqslant P \\ \operatorname{gcd}\left(k_{1}, M z\right)=1}} \sum_{k_{2}\left|\tilde{a} k_{3}\right| \tilde{b}} \sum_{X, Y} \tau(L(X, Y)),
$$

where

$$
L(X, Y)=\varepsilon_{4} \ell_{1} \ell_{1}^{\prime \prime} \ell_{4} \ell_{4}^{\prime \prime} 2^{\delta} \tilde{a}\left[k_{1}, k_{3}\right] Y-\varepsilon_{2} \varepsilon_{3} \ell_{2} \ell_{2}^{\prime \prime} \ell_{3} \ell_{3}^{\prime \prime} 2^{\beta+\gamma} \tilde{b}\left[k_{1}, k_{2}\right] X .
$$

Recall (7.16). Summing over $t_{0} \in T_{M}$, we may bound this contribution using Lemma 2.1 by

$$
\begin{aligned}
& \ll T_{1}^{\varepsilon} \sum_{\substack{\tilde{a} \leqslant P / A \sqrt{T_{2}}<k_{1} \leqslant P \\
\tilde{b} \leqslant P / B}} \sum_{k_{2}\left|\tilde{a}, k_{3}\right| \tilde{b}}\left(\frac{\psi\left(k_{1}\right) \psi(\tilde{a} \tilde{b})^{2} P^{2} \log P}{\left[k_{1}, k_{2}\right]\left[k_{1}, k_{3}\right] C D}+\frac{P^{1+\varepsilon}}{k_{1}}\right) \\
& \ll \frac{T_{1}^{\varepsilon} P^{4}(\log P)^{3}}{A B C D \sqrt{T_{2}}}+P^{3+\varepsilon} .
\end{aligned}
$$

Applying Lemma 7.5 with $R_{1}=1$ and $\boldsymbol{\delta}=(1,1,0)$, this therefore shows that $k_{1}>\sqrt{T_{2}}$ contribute $O\left(P^{4}(\log P)^{3+\varepsilon} / \sqrt{T_{2}}\right)$ to $N_{\mathrm{Br}}(P)$. This is satisfactory for Theorem 1.3, Likewise, the same argument shows that parameters with $\max \left\{k_{2}, k_{3}\right\}>T_{1}$ make a satisfactory overall contribution to $N_{\mathrm{Br}}(P)$. 
Our work so far has therefore shown that we can approximate $\tilde{N}_{1}$ by

$$
\begin{gathered}
\tilde{N}_{2}=\sum_{t_{0} \in T_{M}} \sum_{\substack{\tilde{a} \leqslant P / A, \tilde{b} \leqslant P / B \\
\operatorname{gcd}(\tilde{a}, \tilde{b})=1 \\
(\tilde{a}, \tilde{b}) \equiv\left(a_{0}, b_{0}\right)(\bmod M)}} \sum_{\substack{z \leqslant P^{2} /\left(M T_{2}\right) \\
\operatorname{gcd}(z, \tilde{a} \tilde{b} M)=1}} g_{\mathbf{a}, \mathbf{q}}(z) \\
\times \sum_{\substack{k_{1} \leqslant \sqrt{T_{2}} \\
\operatorname{gcd}\left(k_{1}, M z\right)=1}} \sum_{\substack{k_{2}, k_{3} \leqslant T_{1} \\
k_{2}\left|\tilde{a}, k_{3}\right| \tilde{b}}} \mu\left(k_{1}\right) \mu\left(k_{2}\right) \mu\left(k_{3}\right) \# \mathscr{A}_{z, \mathbf{k}},
\end{gathered}
$$

with acceptable error. To handle $\# \mathscr{A}_{z, \mathbf{k}}$ we call upon Lemma 2.2 , noting that $\# \mathscr{A}_{z, \mathbf{k}}=$ $N(U, V ; \mathbf{a})$ in (2.12), with $(q, r)=(z, M)$ and

$$
\begin{aligned}
& a_{1}=-\varepsilon_{2} \varepsilon_{3} \ell_{2} \ell_{2}^{\prime \prime} \ell_{3} \ell_{3}^{\prime \prime} 2^{\beta+\gamma} \tilde{b}\left[k_{1}, k_{2}\right], \\
& a_{2}=\varepsilon_{4} \ell_{1} \ell_{1}^{\prime \prime} \ell_{4} \ell_{4}^{\prime \prime} 2^{\delta} \tilde{a}\left[k_{1}, k_{3}\right],
\end{aligned}
$$

and furthermore,

$$
U=\frac{P}{\left[k_{1}, k_{2}\right] C}, \quad V=\frac{P}{\left[k_{1}, k_{3}\right] D} .
$$

In particular it is clear that $\operatorname{gcd}\left(a_{1} a_{2} r, q\right)=1$ and so all the conditions are met for an application of Lemma 2.2. This gives

$$
\left|\# \mathscr{A}_{z, \mathbf{k}}-\frac{\varphi(z) P^{2}}{M^{2} z^{2}\left[k_{1}, k_{2}\right]\left[k_{1}, k_{3}\right] C D}\right| \ll \frac{\tau_{3}(z) P}{k_{1} z}+\tau_{3}(z)(\log z M)^{3}+E
$$

where

$$
E=\sum_{d \mid z} d \sum_{\substack{0<|m|,|n| \leqslant M z / 2 \\ m a_{2}-n a_{1} \equiv 0(\bmod d)}} \frac{1}{|m n|} .
$$

We need to sum these three error terms over the remaining parameters to check that they ultimately make a negligible contribution.

The first term on the right of (17.19) is easy to deal with. Lemma 7.5 with $\boldsymbol{\delta}=\mathbf{0}$ and $R_{2}=T_{1}$ easily shows that it contributes $O\left(P^{3+\varepsilon}\right)$ overall. Recall that $\left|g_{\mathbf{a}, \mathbf{q}}(z)\right| \leqslant 1$ and $\tau_{3}(z)$ has average order $(\log z)^{2}$. The contribution to $\tilde{N}_{2}$ from the second term on the right of (7.19) is therefore seen to be

$$
\begin{aligned}
& \ll(\log P)^{5} \sum_{t_{0} \in T_{M}} \sum_{\substack{\tilde{a} \leqslant P / A, \tilde{b} \leqslant P / B \\
\operatorname{gcd}(\tilde{a}, \tilde{b})=1 \\
(\tilde{a}, \tilde{b}) \equiv\left(a_{0}, b_{0}\right)(\bmod M)}} \sum_{\substack{k_{1} \leqslant \sqrt{T_{2}} \\
\operatorname{gcd}\left(k_{1}, M\right)=1}} \sum_{\left.k_{2} \mid \tilde{a}\right)} \frac{P^{2}}{M T_{3} \mid \tilde{b}} \\
& \ll \frac{M P^{2}(\log P)^{5}}{\sqrt{T_{2}}} \sum_{\substack{\tilde{a} \leqslant P / A, \tilde{b} \leqslant P / B\\
}(\tilde{a}) \tau(\tilde{b})} \\
& \ll \frac{M P^{4}(\log P)^{7}}{A B \sqrt{T_{2}}} .
\end{aligned}
$$

We note here that $M / A B \leqslant T_{1}$. Hence once summed over the remaining parameters using Lemma 7.5 with $\boldsymbol{\delta}=\mathbf{0}$ and $R_{2}=T_{1}$, we obtain the satisfactory overall contribution $O\left(T_{1}^{5+\varepsilon} P^{4}(\log P)^{7} / \sqrt{T_{2}}\right)$. 
It remains to handle the contribution to $\tilde{N}_{2}$ from $E$. Let us write $a_{1}=a_{1}^{\prime} \tilde{b}$ and $a_{2}=a_{2}^{\prime} \tilde{a}$. Then since $d \mid z$ it follows that $\operatorname{gcd}\left(a_{1}^{\prime} a_{2}^{\prime}, d\right)=1$. Taking $\left|g_{\mathbf{a}, \mathbf{q}}(z)\right| \leqslant 1$ and carrying out the sums over $k_{1}, k_{2}, k_{3}$ and $t_{0}$, this contribution is seen to be

$$
\ll M^{2} T_{1}^{2} \sqrt{T_{2}} \sum_{\substack{z \leqslant P^{2} /\left(M T_{2}\right) \\ \operatorname{gcd}(z, M)=1}} \sum_{d \mid z} d \sum_{0<|m|,|n| \leqslant M z / 2} \frac{1}{|m n|} \sum_{\substack{\tilde{a} \leqslant P / A, \tilde{b} \leqslant P / B \\ \operatorname{gcd}(\tilde{a} \tilde{b})=1 \\ \operatorname{gcd}(\tilde{b}, z)=1 \\ m a_{2}^{\prime} \tilde{a}-n a_{1}^{\prime} \tilde{b} \equiv 0(\bmod d)}} 1 .
$$

We will need to sort the sum according to the greatest common divisor $h=\operatorname{gcd}(m, n, d)$, writing $\left(m^{\prime}, n^{\prime}, d^{\prime}\right)=(m, n, d) / h$. The final condition on $\tilde{a}, \tilde{b}$ forces the vectors $(\tilde{a}, \tilde{b})$ in which we are interested to lie on a rank 2 integer sublattice of determinant $d^{\prime}$. Heath-Brown [5, Lemma 2] therefore shows that the number of $(\tilde{a}, \tilde{b})$ is

$$
\ll \frac{\operatorname{gcd}(m, n, d) P^{2}}{d A B}+1 .
$$

Substituting this into the above the second term here is seen to contribute

$$
\begin{aligned}
& \ll M^{2} T_{1}^{2} \sqrt{T_{2}}(\log P)^{2} \sum_{z \leqslant P^{2} /\left(M T_{2}\right)} \sum_{d \mid z} d \\
& \ll \frac{P^{4} T_{1}^{2}(\log P)^{2}}{T_{2}^{3 / 2}},
\end{aligned}
$$

to $\tilde{N}_{2}$, which once combined with Lemma 7.5 therefore leads to a satisfactory overall contribution. Finally, the first term contributes

$$
\begin{aligned}
& \ll \frac{M^{2} T_{1}^{2} \sqrt{T_{2}} P^{2}}{A B} \sum_{z \leqslant P^{2} /\left(M T_{2}\right)} \sum_{d \mid z} \sum_{0<|m|,|n| \leqslant M z / 2} \frac{\operatorname{gcd}(m, n, d)}{|m n|} \\
& \ll \frac{P^{4} T_{1}^{3}(\log P)^{3}}{\sqrt{T_{2}}}
\end{aligned}
$$

to $\tilde{N}_{2}$, since $M / A B \leqslant T_{1}$. This too is found to be satisfactory once summed over all the remaining parameters using Lemma 7.5 .

Having handled the contribution from the error terms in (17.19), we are now free to approximate $\# \mathscr{A}_{z, \mathbf{k}}$ by the expected main term. Having done so, furthermore, it is convenient to extend the summations over $k_{1}, k_{2}, k_{3}$ to infinity, which we may do with acceptable error using Lemma 7.5, It henceforth suffices to consider the quantity

$$
\begin{gathered}
\tilde{N}_{3}=\frac{P^{2}}{C D M^{2}} \sum_{t_{0} \in T_{M}} \sum_{\substack{\tilde{a} \leqslant P / A, \tilde{b} \leqslant P / B \\
\operatorname{gcd}(\tilde{a}, \tilde{b})=1 \\
(\tilde{a}, \tilde{b}) \equiv\left(a_{0}, b_{0}\right)(\bmod M)}} \sum_{\substack{z \leqslant P^{2} /\left(M T_{2}\right) \\
\operatorname{gcd}(z, \tilde{a} \tilde{b} M)=1}} \frac{g_{\mathbf{a}, \mathbf{q}}(z) \varphi^{*}(z)}{z} \\
\times \sum_{\substack{k_{1}=1 \\
\operatorname{gcd}\left(k_{1}, M z\right)=1}}^{\infty} \sum_{\substack{k_{2}\left|\tilde{a} \\
k_{3}\right| \tilde{b}}} \frac{\mu\left(k_{1}\right) \mu\left(k_{2}\right) \mu\left(k_{3}\right)}{\left[k_{1}, k_{2}\right]\left[k_{1}, k_{3}\right]},
\end{gathered}
$$


in place of $\tilde{N}_{2}$. We may carry out the summations over $k_{1}, k_{2}, k_{3}$, finding that

$$
\sum_{\substack{k_{1}=1 \\ \operatorname{gcd}\left(k_{1}, M z\right)=1}}^{\infty} \sum_{\substack{k_{2}\left|\tilde{a} \\ k_{3}\right| \tilde{b}}} \frac{\mu\left(k_{1}\right) \mu\left(k_{2}\right) \mu\left(k_{3}\right)}{\left[k_{1}, k_{2}\right]\left[k_{1}, k_{3}\right]}=\frac{6}{\pi^{2} \varphi_{2}^{*}(M z) \psi(\tilde{a} \tilde{b})},
$$

in the notation of (2.10) and (2.11). Hence

$$
\tilde{N}_{3}=\frac{6 P^{2}}{\pi^{2} C D M^{2} \varphi_{2}^{*}(M)} \sum_{t_{0} \in T_{M}} \sum_{\substack{\tilde{a} \leqslant P / A, \tilde{b} \leqslant P / B \\ \operatorname{gcd}(\tilde{a}, \tilde{b})=1 \\(\tilde{a}, \tilde{b}) \equiv\left(a_{0}, b_{0}\right)(\bmod M)}} \frac{1}{\psi(\tilde{a} \tilde{b})} \sum_{\substack{z \leqslant P^{2} /\left(M T_{2}\right) \\ \operatorname{gcd}(z, \tilde{a} \tilde{b} M)=1}} \frac{g_{\mathbf{a}, \mathbf{q}}(z)}{z \psi(z)},
$$

since $\varphi^{*} / \varphi_{2}^{*}=1 / \psi$.

Rather than carrying out the sum over $z$ directly, which intimately depends on $\tilde{a}, \tilde{b}$, we shall first show that $g_{\mathbf{a}, \mathbf{q}}$ can be approximated by the function $\hat{g}_{M}$ defined in (7.3). To this end we exame the sum

$$
\Sigma=\sum_{\substack{\tilde{a} \leqslant P_{1}, \tilde{b} \leqslant P_{2} \\ \operatorname{gcd}(\tilde{a}, \tilde{b})=1 \\(\tilde{a}, \tilde{b}) \equiv\left(a_{0}, b_{0}\right)(\bmod M)}} \frac{1}{\psi(\tilde{a} \tilde{b})} \sum_{\substack{z \leqslant Z \\ \operatorname{gcd}(z, \tilde{a} \tilde{b} M)=1}} \frac{g_{\mathbf{a}, \mathbf{q}}(z)-\hat{g}_{M}(z)}{z \psi(z)},
$$

for given parameters $P_{1}, P_{2} \leqslant P$ and $Z \leqslant P^{2}$. Our aim is to show that

$$
\Sigma \ll P^{2-4 / 11+\varepsilon}
$$

which leads to a satisfactory overall contribution once summed over the remaining parameters, via Lemma 7.5 with $\boldsymbol{\delta}=\mathbf{0}$ and $R_{1}=T_{1}$. To establish the desired bound for $\Sigma$ we deduce from (7.2) and (7.3) that

$$
g_{\mathbf{a}, \mathbf{q}}(z)-\hat{g}_{M}(z)=\frac{\mu(z) \chi_{M}(z)}{2^{\omega(z)}} \sum_{\substack{\ell \mid z \\ \ell>1}}\left(\frac{\tilde{a} \tilde{b} q_{1}}{\ell}\right),
$$

with $q_{1}=\varepsilon_{2} A B$. This allows us to write

$$
\Sigma=\sum_{z \leqslant Z} \frac{\mu(z) \chi_{M}(z)}{2^{\omega(z)} z \psi(z)} \sum_{\substack{\ell \mid z \\ \ell>1}} \sum_{\substack{a c j \leqslant P_{1}, \tilde{b} \leqslant P_{2} \\(\tilde{a}, \tilde{b}) \equiv(\tilde{a}) \equiv\left(a_{0}, b_{0}\right)(\bmod M)}} \frac{1}{\psi(\tilde{a} \tilde{b})}\left(\frac{\tilde{a} \tilde{b} q_{1}}{\ell}\right) .
$$

For $L \leqslant P^{2}$ we let $\Sigma_{1}\left(\right.$ resp. $\left.\Sigma_{2}\right)$ denote the contribution to $\Sigma$ from $\ell \leqslant L$ (resp. $\ell>L$ ).

We begin by estimating $\Sigma_{1}$. For this it will be convenient to write $\psi^{-1}=1 * f$, where $f$ is given multiplicatively at prime powers by

$$
f\left(p^{\nu}\right)= \begin{cases}1, & \text { if } \nu=0 \\ \frac{-1}{p+1}, & \text { if } \nu=1 \\ 0, & \text { otherwise }\end{cases}
$$


For given $\ell, q \in \mathbb{N}$ with $\ell>1$, this allows us to deduce that

$$
\begin{aligned}
\sum_{\substack{n \leqslant x \\
\operatorname{gcd}(n, q)=1}} \frac{1}{\psi(n)}\left(\frac{n}{\ell}\right) & =\sum_{\substack{e \leqslant x \\
\operatorname{gcd}(e, q)=1}} f(e)\left(\frac{e}{\ell}\right) \sum_{\substack{d \leqslant x / e \\
\operatorname{gcd}(d, q)=1}}\left(\frac{d}{\ell}\right) \\
& =\sum_{\substack{e \leqslant x \\
\operatorname{gcd}(e, q)=1}} f(e)\left(\frac{e}{\ell}\right) \sum_{k \mid q} \mu(k)\left(\frac{k}{\ell}\right) \sum_{d \leqslant x / e k}\left(\frac{d}{\ell}\right) \\
& \ll \tau(q) \sqrt{x} \ell^{3 / 16+\varepsilon} \sum_{e \leqslant x} \frac{|f(e)|}{\sqrt{e}} \\
& \ll q^{\varepsilon} \sqrt{x} \ell^{3 / 16+\varepsilon},
\end{aligned}
$$

by the Burgess bound for character sums. Equipped with this it is straightforward to conclude that $\Sigma_{1} \ll P^{\varepsilon} \sqrt{P_{1}} P_{2} L^{3 / 16} \ll P^{3 / 2+\varepsilon} L^{3 / 16}$.

Turning to the contribution from $\ell>L$ and writing $z=\ell z^{\prime}$, we have

$$
\Sigma_{2}=\sum_{z^{\prime} \leqslant Z / L} \frac{\mu\left(z^{\prime}\right) \chi_{M}\left(z^{\prime}\right)}{2^{\omega\left(z^{\prime}\right)} z^{\prime} \psi\left(z^{\prime}\right)} \sum_{\substack{L<\ell \leqslant Z / z^{\prime} \\ \operatorname{gcd}\left(\ell, z^{\prime}\right)=1}} \frac{\mu(\ell) \chi_{M}(\ell)}{2^{\omega(\ell)} \ell \psi(\ell)} \sum_{\tilde{a}, \tilde{b}} \frac{1}{\psi(\tilde{a} \tilde{b})}\left(\frac{\tilde{a} \tilde{b} q_{1}}{\ell}\right) .
$$

Note that $\ell$ is necessarily odd in this summation. Rearranging terms we are led to an inner sum of the form

$$
\sum_{\substack{L<\ell \leqslant Z / z^{\prime} \\ 2 \nmid \ell}} \frac{1}{\ell} \sum_{\tilde{a} \leqslant P_{1}} \alpha_{\ell} \beta_{\tilde{a}}\left(\frac{\tilde{a}}{\ell}\right)
$$

for suitable real numbers $\alpha_{\ell}, \beta_{\tilde{a}}$ with modulus at most 1 . Breaking the $\ell$ sum into dyadic intervals and applying Heath-Brown's large sieve for real characters [6, Cor. 4], we obtain

$$
\begin{aligned}
\Sigma_{2} & \ll P_{2} P^{\varepsilon / 2} \sum_{z^{\prime} \leqslant Z / L} \frac{1}{z^{\prime}} \max _{L<L^{\prime} \leqslant P^{2}}\left\{P_{1}^{1 / 2}+L^{\prime-1 / 2} P_{1}\right\} \\
& \ll P^{3 / 2+\varepsilon}+L^{-1 / 2} P^{2+\varepsilon} .
\end{aligned}
$$

Taking $L=P^{8 / 11}$ and combining this with our estimate for $\Sigma_{1}$, we therefore arrive at the desired bound for $\Sigma$ in (7.21).

Having shown that we may safely approximate $g_{\mathbf{a}, \mathbf{q}}$ by $\hat{g}_{M}$ in $\tilde{N}_{3}$, we proceed to swap the sums over $(\tilde{a}, \tilde{b})$ and $z$. In this way we are led to consider the sum

$$
S=\sum_{\substack{\tilde{a} \leqslant P / A, \tilde{b} \leqslant P / B \\ \operatorname{gcd}(\tilde{a} \tilde{b})=1 \\ \operatorname{gcd}(\tilde{a} \tilde{b}, z)=1 \\(\tilde{a}, \tilde{b}) \equiv\left(a_{0}, b_{0}\right)(\bmod M)}} \frac{1}{\psi(\tilde{a} \tilde{b})} .
$$

The asymptotic evaluation of $S$ is the object of the following result.

Lemma 7.6. - Let

$$
S_{1}=\frac{c_{1} \varphi^{*}(z)^{2} P^{2}}{A B M^{2}} \prod_{p \mid M z} \frac{\left(1+\frac{1}{p}\right)}{\left(1-\frac{1}{p}\right)}\left(1+\frac{2}{p}-\frac{1}{p^{2}}\right)^{-1},
$$


where

$$
c_{1}=\prod_{p} \frac{\left(1-\frac{1}{p}\right)}{\left(1+\frac{1}{p}\right)}\left(1+\frac{2}{p}-\frac{1}{p^{2}}\right) .
$$

Then we have

$$
\sum_{m, n} \sum_{\ell} \sum_{m^{\prime \prime}, n^{\prime \prime}} \sum_{\beta, \gamma, \delta} \sum_{\ell^{\prime \prime}} \frac{P^{2}}{C D M^{2}} \sum_{t_{0}} \sum_{z} \frac{1}{z \psi(z)}\left|S-S_{1}\right| \ll \frac{P^{4}(\log P)^{1+\varepsilon}}{T_{1}},
$$

for any $\varepsilon>0$, where the outer sums are over parameters satisfying (7.16).

Proof. - Since $\tilde{a}$ and $\tilde{b}$ are coprime, we have $\psi(\tilde{a} \tilde{b})=\psi(\tilde{a}) \psi(\tilde{b})$. Writing $\psi^{-1}=1 * f$, where $f$ is given by (7.22), we obtain

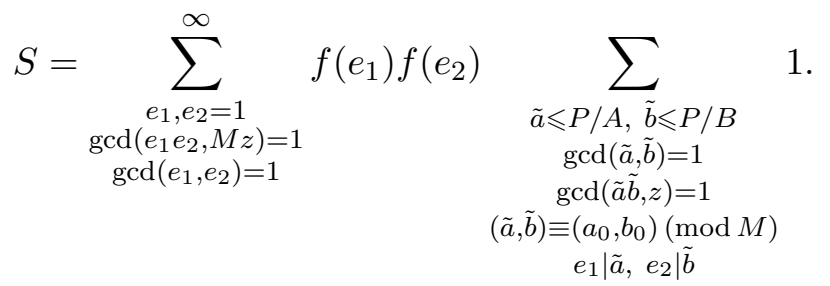

We recall that $\operatorname{gcd}\left(a_{0} b_{0}, M\right)=1$. Making the obvious change of variables, and and using the Möbius function to detect coprimality conditions, the inner sum becomes

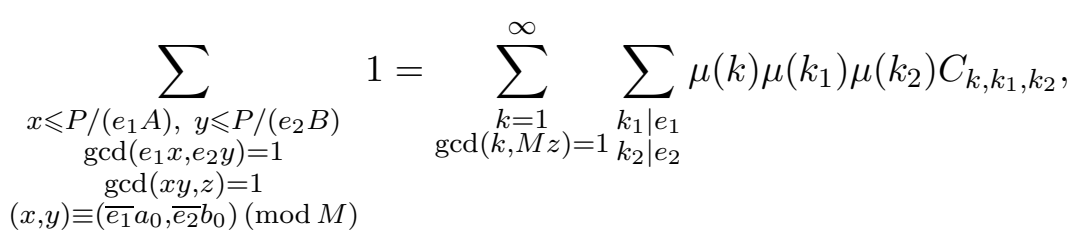

where $C_{k, k_{1}, k_{2}}$ denotes the number of positive integers $x \leqslant P /\left(e_{1} A\right)$ and $y \leqslant P /\left(e_{2} B\right)$ such that

$$
\operatorname{gcd}(x y, z)=1, \quad\left[k, k_{2}\right]\left|x, \quad\left[k, k_{1}\right]\right| y,
$$

and $(x, y) \equiv\left(\overline{e_{1}} a_{0}, \overline{e_{2}} b_{0}\right)(\bmod M)$. Note here that $k_{1}$ and $k_{2}$ are automatically both coprime to $M z$ since $e_{1}$ and $e_{2}$ are. Making a further change of variables, $C_{k, k_{1}, k_{2}}$ is equal to the number of positive integers $x^{\prime} \leqslant P /\left(\left[k, k_{2}\right] e_{1} A\right)$ and $y^{\prime} \leqslant P /\left(\left[k, k_{1}\right] e_{2} B\right)$ such that $\operatorname{gcd}\left(x^{\prime} y^{\prime}, z\right)=1$ and $\left(x^{\prime}, y^{\prime}\right) \equiv\left(x_{0}, y_{0}\right)(\bmod M)$, for integers $x_{0}=\overline{\left[k, k_{2}\right] e_{1}} a_{0}$ and $y_{0}=\overline{\left[k, k_{1}\right] e_{2}} b_{0}$.

Taking a trivial upper bound for $C_{k, k_{1}, k_{2}}$, we find that

$$
\sum_{a_{0}, b_{0}(\bmod M)} C_{k, k_{1}, k_{2}} \ll \frac{P^{2}}{\left[k, k_{1}\right]\left[k, k_{2}\right] e_{1} e_{2} A B} .
$$

Note that

$$
\sum_{e \geqslant E} \frac{|f(e)|}{e} \ll \frac{1}{E}
$$

for any $E \geqslant 1$. In the usual way, combining these estimates with Lemma 7.5 shows that the overall contribution from $\max \left\{k, e_{1}, e_{2}\right\}>T_{1}$ is $O\left(P^{4} T_{1}^{-1+\varepsilon}\right)$, for any $\varepsilon>0$. Hence we may assume that $\max \left\{k, e_{1}, e_{2}\right\} \leqslant T_{1}$ in (7.23).

We estimate $C_{k, k_{1}, k_{2}}$ using Lemma 5.1 twice, with $q_{1}=z$ and $q_{2}=M$. This is valid provided that $P /\left(\left[k, k_{2}\right] e_{1} A\right) \gg T_{1} M z^{\varepsilon}$ and $P /\left(\left[k, k_{1}\right] e_{2} B\right) \gg T_{1} M z^{\varepsilon}$. Recall from (7.8) and 
(7.16) that $A, B \leqslant T_{1}^{5}$ and $M \ll T_{1}^{4}$. It therefore suffices to have $P \gg T_{1}^{13} z^{\varepsilon}$, which obviously holds. Hence we may conclude that

$$
C_{k, k_{1}, k_{2}}=\frac{\varphi^{*}(z)^{2} P^{2}}{\left[k, k_{1}\right]\left[k, k_{2}\right] e_{1} e_{2} A B M^{2}}\left(1+O\left(\frac{1}{T_{1}^{1-\varepsilon}}\right)\right) .
$$

The overall contribution from the error term here, once substituted into (7.23) and summed over the remaining parameters, is easily found to be $O\left(P^{4}(\log P) T_{1}^{-1+\varepsilon}\right)$. Similarly, the shape of the main term allows us to extend the summations over $k$ and $e_{1}, e_{2}$ to infinity with acceptable error. We are therefore led to approximate $S$ by

$$
S_{1}=\frac{\varphi^{*}(z)^{2} P^{2}}{A B M^{2}} \sum_{\substack{e_{1}, e_{2}=1 \\ \operatorname{gcd}\left(e_{2} e_{2}, M z\right)=1 \\ \operatorname{gcd}\left(e_{1}, e_{2}\right)=1}}^{\infty} \frac{f\left(e_{1}\right) f\left(e_{2}\right)}{e_{1} e_{2}} \sum_{\substack{k=1 \\ \operatorname{gcd}(k, M z)=1}}^{\infty} \sum_{\substack{k_{1}\left|e_{1} \\ k_{2}\right| e_{2}}} \frac{\mu(k) \mu\left(k_{1}\right) \mu\left(k_{2}\right)}{\left[k, k_{1}\right]\left[k, k_{2}\right]} .
$$

The inner sum over $k, k_{1}, k_{2}$ here has already been calculated in (7.20). Finally, by calculating Euler factors, one sees that the sums over $e_{1}, e_{2}, k, k_{1}, k_{2}$ evaluate to

$$
\prod_{p \nmid M z}\left(1-\frac{1}{p^{2}}\right)\left(1-\frac{2}{(p+1)^{2}}\right)=\prod_{p \nmid M z} \frac{\left(1-\frac{1}{p}\right)}{\left(1+\frac{1}{p}\right)}\left(1+\frac{2}{p}-\frac{1}{p^{2}}\right) .
$$

It now follows that $S_{1}$ agrees with expression in the statement of the lemma.

Armed with Lemma 7.6, we now return to our expression for $\tilde{N}_{3}$. Let

$$
h(z)=\frac{\varphi^{*}(z)^{2}}{\psi(z)} \prod_{p \mid z} \frac{\left(1+\frac{1}{p}\right)}{\left(1-\frac{1}{p}\right)}\left(1+\frac{2}{p}-\frac{1}{p^{2}}\right)^{-1}=\prod_{p \mid z}\left(1-\frac{1}{p}\right)\left(1+\frac{2}{p}-\frac{1}{p^{2}}\right)^{-1} .
$$

Furthermore, we observe that

$$
\begin{aligned}
\frac{1}{\varphi_{2}^{*}(M)} \prod_{p \mid M} \frac{\left(1+\frac{1}{p}\right)}{\left(1-\frac{1}{p}\right)}\left(1+\frac{2}{p}-\frac{1}{p^{2}}\right)^{-1} & =\prod_{p \mid M}\left(1-\frac{1}{p}\right)^{-2}\left(1+\frac{2}{p}-\frac{1}{p^{2}}\right)^{-1} \\
& =\gamma_{1}(M),
\end{aligned}
$$

say. Then, in place of $\tilde{N}_{3}$, our work so far has shown that we may work with

$$
\tilde{N}_{4}=\frac{6 c_{1} P^{4}}{\pi^{2} A B C D} \times \frac{\gamma_{1}(M) \# T_{M}}{M^{4}} \sum_{\substack{z \leqslant P^{2} /\left(M T_{2}\right) \\ \operatorname{gcd}(z, M)=1}} \frac{\hat{g}_{M}(z) h(z)}{z},
$$

where $c_{1}$ is given in the statement of Lemma 7.6,

We have now arrived at the final stages of the argument, where it is necessary to evaluate the sum over $z$ asymptotically. This is achieved by taking $Z$ of order $P^{2} / M T_{2}$ in Lemma 7.2. In particular it is clear that $\log Z \gg \log P$. Let us write $c_{2}=c_{2}(h)$ and $\gamma_{2}(M)=\gamma(M ; h)$. Since (7.4) clearly holds, we deduce that

$$
\tilde{N}_{4}=\frac{24 c_{1} c_{2} P^{4}}{2^{1 / 4} \pi^{2}|\Gamma(-1 / 4)| A B C D(\log P)^{1 / 4}} \times \frac{\gamma_{1}(M) \gamma_{2}(M) \# T_{M}}{M^{4}}\left\{1+O\left(\frac{1}{(\log P)^{1-\varepsilon}}\right)\right\} .
$$

One notes that $\gamma_{1}(M) \gamma_{2}(M) \ll \log T_{1}$ and $\# T_{M} \ll M^{4}$. Hence the overall contribution from the error term here is satisfactory for Theorem 1.3, once summed over the remaining parameters satisfying (7.16) using Lemma 7.5. Finally, using this result a final time we 
can eliminate (7.16) and so extend the summation over the outer parameters to infinity, with acceptable error. Returning to (7.14), we have therefore shown that the statement of Theorem 1.3 holds, with

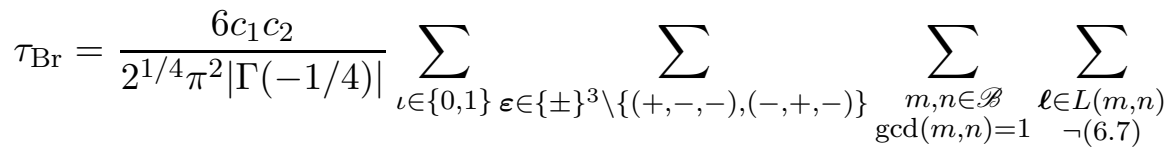

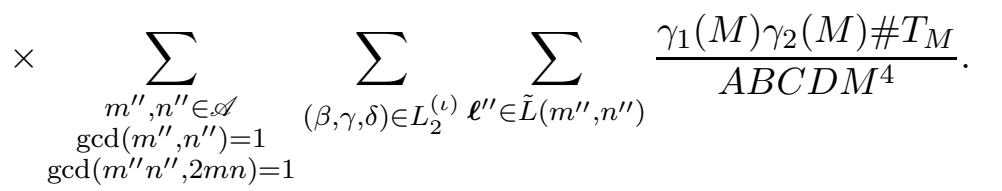

7.3. Evaluation of $\tau_{\mathrm{Br}}$. - We proceed to study this constant, with the goal of showing that it is positive. The set $T_{M}$ was defined to be the $t_{0}(\bmod M)$ for which (7.17) holds. Since $16, m n$ and $m^{\prime \prime} n^{\prime \prime}$ are pairwise coprime, we clearly have

$$
\# T_{M}=\# \tilde{T}_{m n}(\ell) \times \# \tilde{U}_{m^{\prime \prime} n^{\prime \prime}}\left(\ell^{\prime \prime}\right) \times \# \tilde{H}_{\beta, \gamma, \delta} .
$$

Furthermore, Lemma 7.4 implies that

$$
\# \tilde{U}_{m^{\prime \prime} n^{\prime \prime}}\left(\ell^{\prime \prime}\right)=\frac{\varphi\left(m^{\prime \prime} n^{\prime \prime}\right)^{4}}{2^{\omega\left(m^{\prime \prime} n^{\prime \prime}\right)}},
$$

whereas $\# \tilde{T}_{m n}(\ell)$ is given by Lemma 7.3 .

Recall the expression (7.12) for the non-zero constant $\sigma_{2}$. Returning to our formula for the constant, in view of the definition (7.8) of $A, B, C, D$, we sum over the six possible choices for $\varepsilon$ to get

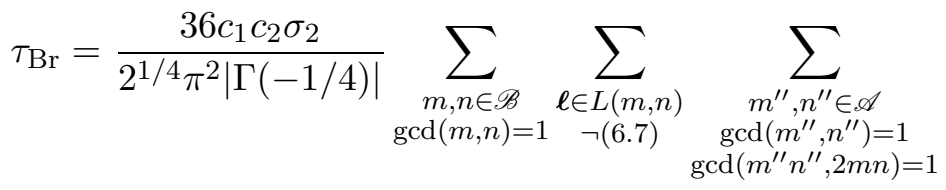

$$
\begin{aligned}
& \times \sum_{\ell^{\prime \prime} \in \tilde{L}\left(m^{\prime \prime}, n^{\prime \prime}\right)} \frac{\gamma_{1}(M) \gamma_{2}(M) \varphi^{*}\left(m^{\prime \prime} n^{\prime \prime}\right)^{4}}{2^{\omega\left(m^{\prime \prime} n^{\prime \prime}\right)} m^{2} n^{2} m^{\prime \prime 2} n^{\prime \prime 2} \ell_{1} \cdots \ell_{4} \ell_{1}^{\prime \prime} \cdots \ell_{4}^{\prime \prime}} \cdot \frac{\# \tilde{T}_{m n}(\ell)}{(m n)^{4}} .
\end{aligned}
$$

Next we undertake the summation over $\ell^{\prime \prime}$, where $\tilde{L}\left(m^{\prime \prime}, n^{\prime \prime}\right)$ is given by (7.10). A simple calculation reveals that

$$
\sum_{\ell^{\prime \prime} \in \tilde{L}\left(m^{\prime \prime}, n^{\prime \prime}\right)} \frac{1}{\ell_{1}^{\prime \prime} \cdots \ell_{4}^{\prime \prime}}=\frac{2^{\omega\left(m^{\prime \prime} n^{\prime \prime}\right)}}{m^{\prime \prime} n^{\prime \prime} \varphi_{2}^{*}\left(m^{\prime \prime} n^{\prime \prime}\right)} .
$$

Likewise, with reference to the definition (5.8) of $L(m, n)$ and Lemma 7.3 , it is straightforward to check that the sum over $\ell$ evaluates to

$$
\begin{aligned}
\sum_{\substack{\ell \in L(m, n) \\
\neg \sqrt{6.7]}}} \frac{1}{\ell_{1} \cdots \ell_{4}} \cdot \frac{\# \tilde{T}_{m n}(\ell)}{(m n)^{4}} & =\varphi^{*}(m n)^{4} \prod_{p \mid m n} \sum_{\boldsymbol{\nu} \in E\left(v_{p}(m), v_{p}(n)\right)} \frac{2^{\tau(\boldsymbol{\nu})-1}}{p^{\nu_{1}+\nu_{2}+\nu_{3}+\nu_{4}}} \\
& =\varphi^{*}(m n)^{4} \gamma_{3}(m n),
\end{aligned}
$$


where $E(i, j)$ is given by (6.12) and

$$
\gamma_{3}(m n)=\prod_{p \mid m n}\left(1-\frac{1}{p}\right)^{-1}\left(1+\frac{1}{p}+\frac{1}{p^{2}-1}\left\{1+\frac{3}{p}-\frac{1}{p^{2}}-\frac{1}{p^{3}}\right\}\right) .
$$

Noting that $\gamma_{1}(16)=\frac{16}{7}$ and $\gamma_{2}(16)=1$, we may therefore conclude that

$$
\begin{aligned}
\tau_{\mathrm{Br}}= & \frac{576 c_{1} c_{2} \sigma_{2}}{2^{1 / 4} 7 \pi^{2}|\Gamma(-1 / 4)|} \sum_{\substack{m, n \in \mathscr{B} \\
\operatorname{gcd}(m, n)=1}} \frac{\gamma_{1} \gamma_{2} \gamma_{3}(m n) \varphi^{*}(m n)^{4}}{m^{2} n^{2}} \\
& \times \sum_{\begin{array}{c}
m^{\prime \prime}, n^{\prime \prime} \in \mathscr{A} \\
\operatorname{gcd}\left(m^{\prime \prime}, n^{\prime \prime}\right)=1 \\
\operatorname{gcd}\left(m^{\prime \prime} n^{\prime \prime}, 2 m n\right)=1
\end{array}} \frac{\gamma_{1} \gamma_{2}\left(m^{\prime \prime} n^{\prime \prime}\right) \varphi^{*}\left(m^{\prime \prime} n^{\prime \prime}\right)^{4}}{m^{\prime \prime 3} n^{\prime \prime 3} \varphi_{2}^{*}\left(m^{\prime \prime} n^{\prime \prime}\right)} .
\end{aligned}
$$

These sums over $m, n, m^{\prime \prime}, n^{\prime \prime}$ are absolutely convergent and positive, as one checks by considering the associated Euler products. This therefore shows that $\tau_{\mathrm{Br}}>0$, as required to complete the proof of Theorem 1.3.

\section{References}

[1] M. Bhargava, Most hyperelliptic curves over $\mathbb{Q}$ have no rational point. Submitted, 2013. (arXiv:1308.0395)

[2] R. de la Bretèche and T.D. Browning, Sums of arithmetic functions over values of binary forms. Acta Arith. 125 (2006), 291-304.

[3] J.-L. Colliot-Thélène, D. Coray and J.-J. Sansuc, Descente et principe de Hasse pour certaines variétés rationnelles. J. reine angew. Math. 320 (1980), 150-191.

[4] J.-L. Colliot-Thélène, J.-J. Sansuc and P. Swinnerton-Dyer, Intersections of two quadrics and Châtelet surfaces, I. J. reine angew. Math. 373 (1987), 37-107; II. ibid. 374 (1987), 72-168.

[5] D.R. Heath-Brown, Diophantine approximation with square-free numbers. Math. Zeit. 187 (1984), $335-344$.

[6] D.R. Heath-Brown, A mean value estimate for real character sums. Acta Arith. 72 (1995), 235275 .

[7] V.A. Iskovskikh, A counterexample to the Hasse principle for systems of two quadratic forms in five variables. (Russian) Mat. Zametki 10 (1971), 253-257.

[8] P. Le Boudec, Affine congruences and rational points on a certain cubic surface. Submitted, 2012. (arXiv: 1207.2685)

[9] B. Poonen and J.F. Voloch, Random Diophantine equations. Arithmetic of higher-dimensional algebraic varieties (Palo Alto, CA, 2002), 175-184, Progr. Math. 226, Birkhäuser, 2004.

[10] G. Tenenbaum, Introduction to analytic and probabilistic number theory. CUP, 1995.

September 22, 2018

R. De la Bretèche, Institut de Mathématiques de Jussieu, Université Denis Diderot, Case Postale 7012, F75251 Paris CEDEX 13, France • E-mail : breteche@math.jussieu.fr

T.D. Browning, School of Mathematics, University of Bristol, Bristol, BS8 1TW, United Kingdom E-mail : t.d.browning@bristol.ac.uk 